\title{
Spectres d'absorption ultra-violets de quelques cétènes et de leurs dimères
}

\section{Doctoral Thesis}

\section{Author(s):}

Lardy, Guillaume C.

Publication date:

1924

Permanent link:

https://doi.org/10.3929/ethz-a-000091704

Rights / license:

In Copyright - Non-Commercial Use Permitted 
Travail fait au laboratoire de chimie générale de l'E. P. F.

(Professeur D* Staudinger)

et au laboratoire de chimie physique de l'Universitẻ de Zurich

(Professeur $\mathrm{D}^{\text {r VICTOR HENRI) }}$

\title{
SPECTRES D'ABSORPTION ULTRA-VIOLE
do
quelques Cótènes et de leurs Dimères
}

\section{THÈSE}

présentio

A L'ÉCOLE POLYTECHNIQUE FÉdÉRALE DE ZURICH

pour l'obtention du

GRADE DE DOCTEUR ÈS SCIENCES TECHNIQUES

\author{
Psa \\ Guillaume C. LARDY
}

de Neuchâtel

Rapporteur : M. le Professeur $D^{r}$ H. STAUDINGER

Co-Rapporteur : M. lo Profosecur Dr E. BAOR

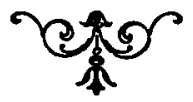

THESE $N^{\circ} 345$

PARIS ( $\left.\mathrm{V}^{\circ}\right)$

LES PRESSES UNIVERSITAIRES DE FRANCE

49. Boulevard Saint-Michel, 49

1924 
Ce travail a été entrepris avec l'approbation de Monsieur le

\section{$D^{r}$ H. STAUDINGER}

Professeur de Chimie générale à l'E. P. F.

Je tiens à le remercier très chaudement de tous les conseils qu'il a bien voulu me donner et de tout l'intérêt qu'il a porté à mes expériences. La préparation des produits a été faite dans ses laboratoires, ainsi que tout le travail de chimie organique.

Les mesures spectrographiques ont été exécutées au laboratoire de chimie physique de l'Université de Zurich. Je suis heureux de pouvoir dire ici toute ma reconnaissance à mon très honoré et très cher maître le

\section{$D^{r}$ Victor HENRI}

\section{Professeur à l'Université de Zurich}

pour toute la peine qu'il s'est donné d wre faire connaître les méthodes de travail spectrographique dont il est lauteur. J'ai été très sensible à lamitié qu'il a bien voulu me témoigner. 
En hommage respectueux

a

ma fancée, Mademoiselle Anny CAFLISCH

et d

mes chers parents 


\title{
SPECTRES D'ABSORPTION ULTRA-VIOLETS
}

\author{
DE QUELQUES CÉTÈNES ET DE LEURS DIMÈRES
}

\section{P REF A E}

\section{GENERALITES SUR LES SPECTRES D'ABSORPTION ET LES CETENES}

1. Spectres d'absorption, degré de non-saturation et labilité des molécules. - Les cétènes se distinguent des autres combinaisons organiques par trois caractères : ils ont une structure simple, ils sont très peu stables, quelques-uns sont colorés. Leur labilité est même si grande que toute réaction transforme la molécule entière. On ne saurait parler d'une réaction du groupe $\mathrm{C}=\mathrm{O}$ ou d'une réaction de la liaison éthylénique. On parle de réactions des cétènes.

Staudinger ( ${ }^{1}$ ) a étudié leurs propriétés dans tous les détails et a observé que les cétènes colorés étaient plus actifs que les autres, que leurs réactions se produisaient d'autant plus rapidement que la couleur était plus foncée. Il a généralisé cette observation, car non seulement les cétènes mais aussi beaucoup d'autres combinaisons chimiques, les $a$ - dicétones, les thiocétones, les composés diazoïques possèdent à première observation cette propriété.

Ceci l'a conduit à énoncer la proposition suivante : Toute substitution chimique qui renforce la couleur d'une combinaison contenant un ou plusieurs chromophores, diminue l'état de saturation de ceux-ci. .

Pour connaître dans quelles limites cette hypothèse se trouve confirmée par l'expérience, nous avons entrepris l'étude des spectres d'absorption des cétènes.

Nous avons observé que les cétènes possèdent un spectre caractéristique, dont une partie se rapproche de celui de l'acétone et l'autre de celui des composés éthyléniques. L'intensité d'absorption ainsi que la position des bandes dépend de la substitution.

Il n'existe pas de différence essentielle entre les aldocétènes, $\mathrm{H}_{2} \mathrm{C}=\mathrm{C}=\mathrm{O}$,

(1) Staudinger, Les Cétènes, Stuttgart, chez Encke, 1912, p. 98.

Guillaume C. Lardy. 
qui sont incolores et les cétocétènes, $\mathrm{R}_{2} \mathrm{G}=\mathrm{C}=\mathrm{O}$ colorés. La couleur provient du déplacement d'une des bandes vers les régions du spectre visible.

La position d'une bande est caractéristique pour les propriétés'd'un cétène; elle dépend de son état énergétique. C'est ce que nous exprimons en chimie par son degré de non-saluration.

Quant à la labilité même des chromophores elle est mesurée par la vitesse de réaction c'est-à-dire par le nombre de transtormations par unité de temps. Cette labilité est représentée par l'intensité de la bande d'absorption caractéristique du chromophore.

C'est la loi que Victor Henri a énoncé en $1913\left({ }^{2}\right)$ :

"Les corps organiques qui réagissent le plus facilement, qui sont les plus instables, possèdent les bandes-ultra-violettes les plus fortes.

D'après la théorie de Bohr nous pouvons considérer la position d'une bande comme liée à l'état des molécules.

La fréquence d'une radiation émise ou absorbée par un système vibrant est proportionnelle au changement d'énergie.

$$
h v=\mathrm{E}_{1}-\mathrm{E}_{2}
$$

où $\mathbf{E}_{1}$ et $\mathrm{E}_{2}$ représentent deux états d'énergie, $h$ la constante universelle de Planck et $y$ la fréquence de la radiation émise ou absorbée.

Dans le cas d'une molécule, l'énergie nécessaire pour effectuer une transformation est mesurée par la fréquence de la bande d'absorption caractèristique de cette molécule. Il existe donc une relation entre l'énergie interne, qui dépend des forces qui agissent entre les atomes et les groupes d'atomes, ef la longueur d'onde de la lumière émise ou absorbée.

Cette relation est de la forme :

$$
\frac{C}{\lambda}=\frac{f(E)}{h}=v
$$

où $C$ est la vitesse de la lumière $\lambda$ la longueur d'onde, $E$ l'énergie interne et $f$ une fonction plus ou moins complexe.

Nous devons conclure que, pour les propriétés chimiques, plus la position de la bande d'absorption se troure vers le rouge plus le corps auquel elle appartient est non-saturé. Dans ce cas l'énergie nécessaire pour faire passer ce corps d'un étal à un autre est petite.

L'intensité de l'émission ou de l'absorption est proportionnelle au nombre de passages d'un état à un autre ; ce nombre dépend de la probabilité de cette transformation. Dans une combinaison la hande d'un chromophore sera d'autant plus intense que celui-ci est plus actif. Nous ne connaissons pas la relation exacte entre l'intensité d'absorption et l'activité chimique, car nous ne possédons qu'une définition théorique de celle-ci. Aucune détermination expérimentale n'en a été faite.

n Le potentiel ou l'affinité chimique est mesure, selon R. Marcelin ( $\left.{ }^{2}\right)$, au

(1) C. R., t. 156, p. 1979, et Etudes de Pkotochimie, Paris, 1919, p. 181.

(2) R. Marcelin, Annales de Physique, 9 ; 3, p. 134 (1915). 
signe près par la variation d'énergie utilisable divisée par la quantité de substance retranchée, pour extraire d'une phase une des substances qui la compose en laissant la masse homogène et sans changer ni sa température ni son volume. "

Si donc nous prenons le terme de renforcement de la couleur dans le sens que lui donnent les chimistes des matières colorantes, il correspond à ce que nous avons désigné par déplacement d'une bande d'absorption. Il dépend du degré de nonsaturation de la molécule.

La signification de la loi énoncée par Staudinger correspond au déplacement de la bande d'absorption - plus la bande se rapproche du visible, plus le corps es instable.

D'autre part la loi énoncée par Victor Henri se rapporle à l'intensité de l'absorption, elle signifie que plus une bande est intense plus un corps est actif - ou comne Victor Henri l'avait exprimé - plus ses molécules sont labiles.

On voit que ces deux lois expriment les deux faces du problème de la stabilité des combinaisons chimiques et se complètent mutuellement. Elles ont été énoncées en 1912 et 1913 c'est-à-dire avant la théorie de Bohr. Grâce à celle-ci nous comprenons aujourd'hui la signification physique de ces lois qui sont en accord parfait avec les théories modernes de Bohr, Sommerfeld et d'autres.

L'étude des spectres d'absorption des cétènes présentait un intérêt tout particulier pour arriver à une connaissance plus approfondie des relations qui existent entre eux.

La grande analogie chimique qu'ils ont avec les isocyanates, les composés diazoïques et les dérivés de l'acide azothydrique, a été mise en évidence par Staudinger ( $\left.{ }^{1}\right)$. Il les groupe sous deux noms nouveaux : les "Azènes ", comprenant les dérivés du chomophore $=\mathbf{N} \equiv \mathbf{N}$ et les "Carbonylènes " avec les dérivés de $=\mathrm{G}=\mathrm{O}$. Nous avons comparé les spectres d'absorption des uns et des autres et sommes parvenus aux mêmes conclusions.

2. Action mutuelle de plusieurs chromophores dans une molécule. Bandes larges et étroites. - Le déplacement d'une bande d'absorption par l'introduction d'un radical chimique dans une molécule n'est pas une proprièté particulière aux cétènes. C'est un phénomène général que Victor Henri a énoncé dans ses deux pre. mières lois sür la répartition des bandes (') :

1. Chaque chromophore possède une bande caractéristique.

20 Lorsque la molécule d'un corps contient deux groupes chromophores elle possède les bandes caractéristiques de ces chromophores. Si ces groupes sont voisins l'un de l'autre dans la molécule, la position des bandes d'absorption est déplacée vers le rouge, l'intensité de l'absorption n'étant que faiblement modifiée. Si ces chromophores sont éloignés les uns des autres dans la molécule. la position des bandes n'est pas modifiée, mais la valeur de l'absorption est augmentée.

(1) H. Staudinger, Helo. Chem. Acta, V ; p. 87 (1922).

(2) Victor Henri, Etudes de Photochimie, 1919, Paris, p. 173. 
Par l'expérience chimique nous savons qu'une molécule qui possède plusieurs chromophore est d'autant moins saturée que ceux-ci sont plus rapprochés les uns des autres. Cetle non-saturation atteint un maximum, lorsque deux chromophores sont directement liés en position cumulée.

Les différentes combinaisons possibles, depuis celles où les chromophores sont très éloignés l'un de l'autre, jusqu'à la cumulation sont représentées par des espèces chimiques bien définies. Nous citerons comme exemple de chacune, les combinaisons desmotropes, les combinaisons à doubles liaisons conjuguées de Thiele et enfin les azènes et les carbonylènes.

V. Henri a énoncé la relation qui existe entre la position des chromophores dans une molécule et son état physique après de nouvelles observations sur la structure des bandes spectrales des vapeurs ou des gaz. Celles-ci sont larges lorsque les chromophores sont éloignés les uns des autres. A mesure qu'ils se rapprochent et qu'une action réciproque prend naissance, la bande se divise en bandes étroites. Enfin une structure fine apparaît. La distribution de ces bandes fines est définie par les lois des spectres de bandes $\left(^{1}\right)$.

D'après le premier postulat de la théorie de Bohr on admet qu'une molécule possède une série d'états stationnaires $E_{1}, E_{2}$, etc... dont l'énergie est déterminée par les mouvements quantifiés des électrons $\varepsilon$, des atomes do et des molécules $\Re$.

$$
\begin{aligned}
& \mathbf{E}_{1}=\varepsilon_{1}+\mathfrak{b}_{1}+M_{b_{1}} \\
& \mathbf{E}_{2}=\varepsilon_{2}+\mathfrak{b}_{2}+M_{2}
\end{aligned}
$$

Le second postulat de Bohr définit la relation entre les changement d'énergie d'un système en vibration et la radiation émise ou absorbée :

$$
\nu=\frac{\mathbf{E}_{1}-\mathbf{E}_{2}}{h}=\frac{\left(\varepsilon_{1}-\varepsilon_{2}\right)+\left(\mathscr{A}_{1}-\mathscr{A}_{2}\right)+\left(M_{1}-M_{b_{2}}\right)}{h}
$$

ce qui donne pour la distribution des bandes spectrales trois possibilités :

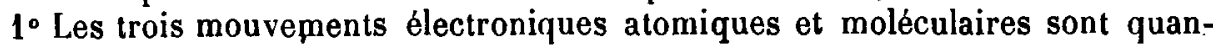
tifiés. Le spectre possède une structure finie.

Exemple : Les spectres de vapeur du benzène, du toluène et du chlorure de benzène.

$2^{\circ}$ Seuls les mouvements électroniques et atomiques sont quantifiés. Le spectre de vapeur possède une structure fine mais plus grossière et surtout moins nette que celle des corps qui précèdent :

Exemple : Spectres de vapeurs du diacétyle et du cétène.

$3^{\circ}$ Seuls les mouvements électroniques sont quantifiés. Le spectre de vapeur présente quelques larges bandes réparties sur toute la longueur du spectre. Exemple : Acétone.

Comme la condition nécessaire à la quantification d'une vibration est l'hétéropolarité des particules en mouvement, Victor Henri en a conclu que $\left({ }^{2}\right)$ : 211.

(1) Victor Henri, Journal de Physique el le Radium, juin 1922, série VI, tome III, pages 181 -

(2) C. R., t. 176, p. 1142 (1923). 
"Pour les molécules ne contenant qu'une seule liaison double, le premier postulat de Bohr ne s'applique pas; seul le second est valable. Pour les molécules à deux liaisons doubles voisines, les deux postulats s'appliquent, le premier étant déterminé par l'existence d'une polarité dans la molécule. o

Par des considérations d'ordre purement chimique L. M. Lowry est arrivé à des conciusions analogues sur la polarité électrique des molécules contenant deux liaisons doubles $\left({ }^{2}\right)$.

Grâce à elles nous pouvons émettre de nouvelles hypothèses sur les réactions des cétènes, qui nous semblent rendre avec exactitude les faits expérimentaux.

3. Polarité électrique de la molécule des cétènes. - Une différence importante entre les électrolytes dissociés et les substances organiques non conductrices, réside dans la marche des réactions.

Broenstedt énonce cela de la façon suivante $\left({ }^{2}\right)$ :

"On constate toujours une anomalie dans la marche d'une réaction lorsque celle-ci a lieu entre des ions, tandis que si. elle a lieu entre des non-électrolytes ou entre ceux-ci et des ions elle est normale ; la loi d'action des masses est directement applicable. "

Les cétènes, tout en étant des composés organiques non-conducteurs présentent des vilesses de réactions plus grandes que la plupart des autres dérivés du carbone. La marche de leurs réactions est toujours anormale. On rencontre dans chacune des phénomènes d'autocatalyse. Ils semblent occuper une place intermédiaire.

Par la théorie cinétique nous savons que le nombre de chocs entre les molécules d'un gaz ou d'un corps dissous est déterminé pour une température et une pression ou concentration donnée. Si les vitesses de réaction étaient proportionnelles aux nombres de collisions, elles devraient toutes être égales entre elles dans les mémes conditions extérieures. C'est loin d'être le cas. Nous supposons donc que seules certaines collisions peuvent donner naissance à la réaction. Ce sont celles qui possèdent un certain minimum d'énergie, auquel Marcelin donne le nom "d'énergie critique de réaction $\left({ }^{3}\right)$. ")

Pour les électrolytes le nombre de collisions actives est très grand ce qui est probablement dù à leur polarité. Chez les non-électrolytes seule une petite proportion des chocs est effective à cause de leur neutralité.

Pour les cétènes cette proportion est plus grande et nous pouvons admettre qu'ils ont des caractères communs avec les ions.

Le cétène, $\mathrm{H}_{2} \mathrm{C}=\mathrm{C}=\mathrm{O}$, possède à l'état de vapeur un spectre de bandes fines. Nous en concluons, d'après la loi de Victor Henri (voir page 4), que sa molécule, comme celle de ses dérivés, est un dipole. Ce qui dans leur molécule est

(1) Jour. Chem. Society, vol. 123, p. 822 (1923).

(2) Zeit. Phys. Chem., no 102, p. 169 (1922).

(8) R. Marcelin, Annales de Physique, 9; 3, p. 168-175 (1915). 
commun avec les ions c'est l'existence de charges électriques localisées à deux endroits différents de la molécule. Par exemple:

$$
\mathrm{H}_{2} \mathrm{C}=\mathrm{G}=\mathrm{O}
$$

La molécule d'un cétène se distingue d'un ion par la présence de deux charges dans une particule, ce qui neutralise l'effet de chacune.

La vitesse de ses réactions doit être inférieure à celle des électrolytes car seules les collisions, où un pôle de l'une rencontre le pôle de signe contraire de l'autre, pourront être suivies d'une réaction.

Ceci se passe lorsque du cétène, polymérise.

$$
\begin{aligned}
& \mathrm{R}_{2}=\mathrm{C}=\mathrm{C}=\mathrm{O} \\
& \mathrm{O}=\overline{\mathrm{C}}=\stackrel{+}{\mathrm{C}}=\mathrm{R}_{2} \\
& \begin{array}{l}
\mathrm{R}_{2}=\mathrm{C}-\mathrm{C}=0 \\
\mathrm{O}=\mathrm{C}-\mathrm{C}=\mathrm{R}_{\mathbf{2}}
\end{array}
\end{aligned}
$$

Les produits de polymérisation, dérivés du dicétocyclobutane nous prouvent bien que les pôles se trouvent aux atomes de carbone.

Le cétène monomoléculaire à température ordinaire n'est qu'une forme labile d'un composé $\left(\mathrm{R}_{2} \mathrm{C}=\mathrm{G}=0\right)_{n}$, où $n$ selon la température est égal à 2 ou 1 .

Selon les propriétés chimiques des radicaux qui le forment l'ensemble est plus ou moins stable les forces de liaisons plus ou moins intenses. Mais on ne peut pas conclure de cette instabilité, comme cela a été fait par Schrocter ( $\left(^{(}\right)$, que les forces qui.lient deux molécules de cétène sont d'une autre nature que celles qui lient les atomes. On ne peut donc supposer sans autres preuves que les dimères des cétènes sont des combinaisons " moléculaires ".

Si d'autre part la caractéristique d'une combinaison dite " moléculaire " est de ne pas posséder de points d'attache bien définis entre ses parties, les dimères des cétènes ne l'ont pas. La conception dipôle des cétènes, que nous devons à Victor Henri, comme la structure dicétonique des dimères, exige que quatre points, ceux où se trouvent les charges, soient liés ensemble dans le dimère.

Nous avons essayé de donner aux deux phénomènes de l'accroissement de l'intensité et du déplacement d'une bande une signification chimique. Nous ne pouvons pas encore dire comment il faut rapporter les valeurs du coefficient d'absorption et de position des bandes, que nous avons trouvées, à d'autres grandeurs physiques caractéristiques pour la molécule (forme de la molécule, distance entre les atomes, grandeurs des charges, moments d'inertie,... etc.).

Elles ont pour le chimiste un autre intérêt. Il peut y reconnaître l'effet d'une

(1) $B ., 49 ; 2697$ (1916). 
substitution sur les propriétés d'une substance, prévoir et calculer la masse du groupe qu'il doit introduire pour obtenir une propriété déterminée.

Ceci peut éviter de longs et nombreux tâtonnements dans une synthèse.

Nous savons que pour les matières colorantes les propriétés sont liées à la présence de certains radicaux. Il en est de même pour certains effets physiologiques. Après avoir étudié les spectres de telles combinaisons et leur variation pour certaine substitution, on peut en déduire les groupes qu'il faut introduire pour obtenir une action particulièrement intense.

A ce point de vue l'étude des spectres d'absorption, qui semble encore un luxe pour le praticien, devient un auxiliaire précieux.

C'est ainsi que nous croyons que la spectrographie peut rendre dès aujourd'hui des services à la chimie industrielle. 
Leer - Vide - Empty 


\title{
PARTIE THÉORIQUE
}

\author{
CHAPITRE PREMIER
}

\section{LES AZ ENES ET LES CARBONYLENES}

Si nous comparons entre eux les corps qui possèdent deux chromophores en position cumulée, nous observons une grande analogie quelle que soit la nature des atomes du radical non saturé.

Ce travail a déjà été entrepris il y a quelques années par Staudinger et ses collaborateurs. Il a porté sur l'élude :

10 Des composés diazoïques ;

$2^{\circ}$ Des cétènes ;

$3^{\circ}$ Des dérivés de l'acide azothydrique, les azides ;

$4^{\circ}$ Des isocyanates.

Staudinger les groupes comme suit $\left(^{2}\right)$ :

\begin{tabular}{|c|c|c|c|}
\hline \multicolumn{2}{|l|}{ Les Axdnee ( $\left.{ }^{2}\right)$} & \multicolumn{2}{|l|}{ 2) Les carbonylènes } \\
\hline $\begin{array}{l}\mathbf{H}_{2} \mathrm{C}=\mathbf{N} \equiv \mathbf{N} \ldots \ldots \ldots \ldots \ldots \ldots \ldots \ldots \\
\left(\mathbf{C H}_{3}\right)_{2} \mathbf{C}=\mathbf{N} \equiv \mathbf{N} \ldots \ldots \ldots \ldots \ldots \ldots \ldots \\
\left(\mathbf{C}_{6} \mathbf{H}_{5}\right)_{2} \mathbf{C}=\mathbf{N} \equiv \mathbf{N} \ldots \ldots \ldots \ldots \ldots \ldots\end{array}$ & $\begin{array}{l}\text { janne } \\
\text { rouge } \\
\text { bleu-rouge }\end{array}$ & 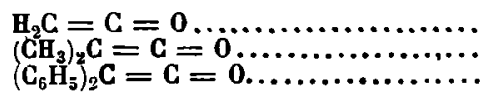 & $\begin{array}{l}\text { incolore } \\
\text { jaune } \\
\text { orange }\end{array}$ \\
\hline 3) & & 4) & \\
\hline 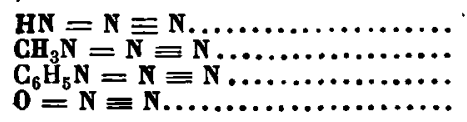 & $\begin{array}{l}\text { incolore } \\
\text { incolore } \\
\text { jaune-vert } \\
\text { incolore }\end{array}$ & 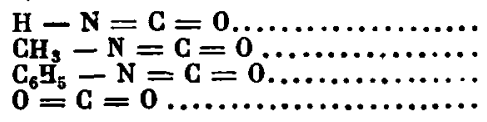 & $\begin{array}{l}\text { incolore } \\
\text { incolore } \\
\text { incolore } \\
\text { incolore }\end{array}$ \\
\hline
\end{tabular}

(1) Helo. Chim. Acta, V, p. 87 (1922).

(अ) Nous devons toutes les données spectrographiques que nous publions sur les dérivés du chromophore $=\mathrm{N} \equiv \mathrm{N}$ à une communication particulière de $\mathrm{M}$. Braunholzer. Nous l'en remercions. C'est dans le laboratoire de M. Victor Henri qu'ont été faites les mesures des spectres. 
La comparaison y est basée sur les réactions chimiques. Elle tend aux mêmes conclusions que celles que nous tirons des spectres d'absorption. La différence qui existe entre les carbonylènes et les azènes d'une part et les carbonylènes et azènes entre eux d'autre part réside dans leur stabilité. Elle pourrait être déterminée par des mesures de vitesses de réactions à différentes températures. Nous parvenons au même résultat par la comparaison des spectres d'absorption.

Tableau donnant la position des maxima et l'intensité des bandes d'absorptions pour les azènes et les carbonylènes ()

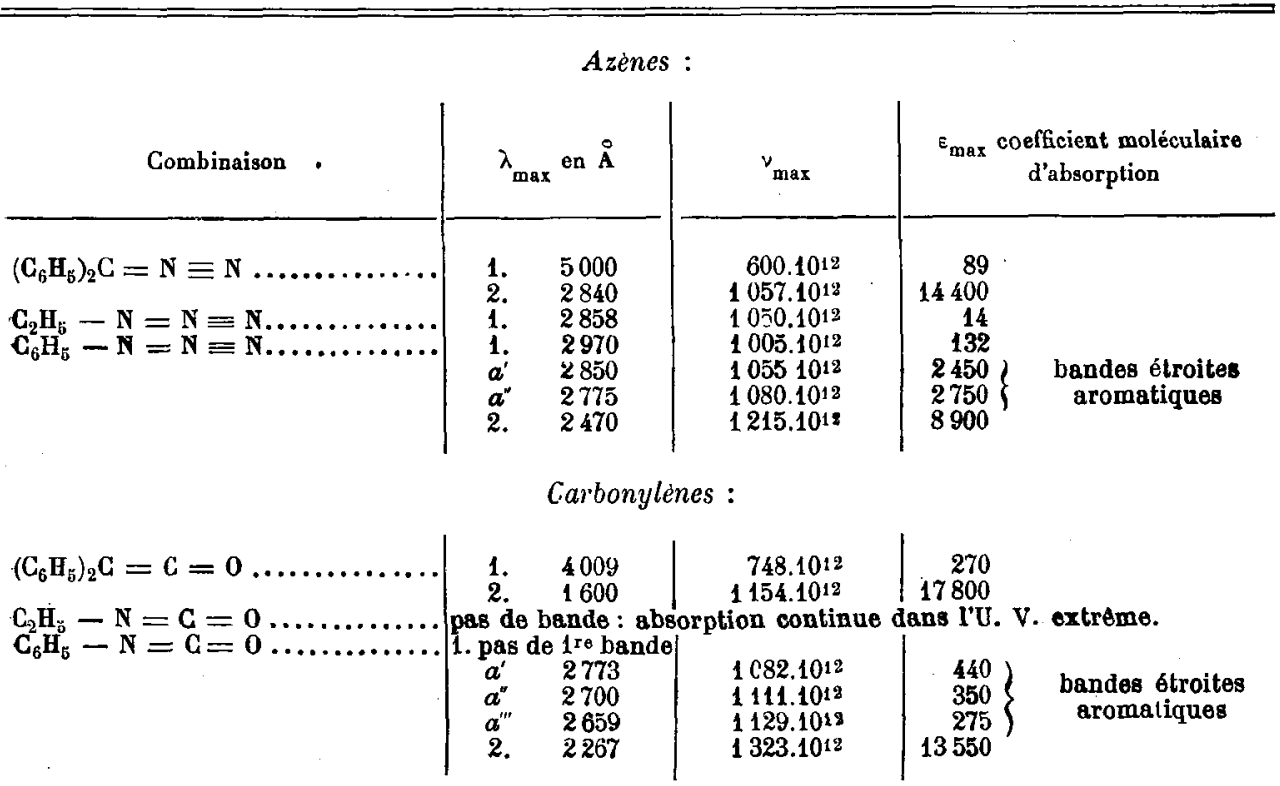

Si nous examinons les spectres des diazoïques et des cétènes, nous observons une grande analogie. Chacun présente deux bandes, l'une très intense dans l'ultraviolet, l'autre à la limite du visible. Ces corps sont bien semblables.

La première bande ressemble à celle de l'acétone $\left(\nu_{\max }=1109.10^{12}\right.$ et $\varepsilon_{\max }=15,8$ d'après Victor Henri) mais est déplacée vers le visible. Ce déplacement est proportionnel á l'action du second chromophore $-=\mathrm{C}=\mathrm{C}=$ pour les cétènes et $=\mathrm{G}=\mathrm{N} \equiv$ pour les diazoïques - sur le premier.

La seconde bande dans l'ultra-violet dépend du second chromophore $>\mathrm{C}=\mathrm{G}<$ ou $>\mathrm{G}=\mathrm{N}<$ et de ses substituants.

(1) La similitude des spectres des cétènes et des diazoïques enlève leur force aux arguments que Hantsch donne en faveur de la notation de Curtius pour les dérivés de $=N \equiv N$. B., 45, p. 3017 (1912). 


Azínes

1 re Bande

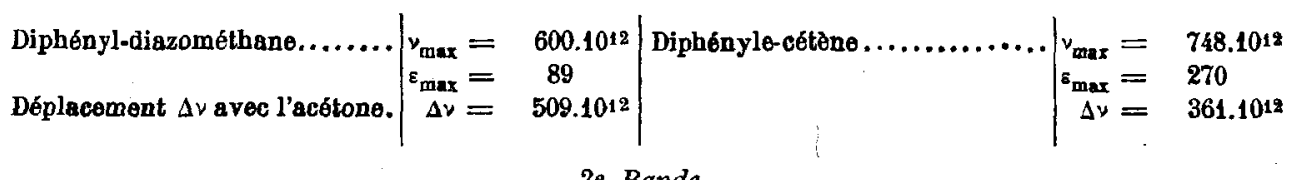

2e Bande

Diphényle-diazométhane....... $\left|\begin{array}{l}\nu_{\max }=1057.10^{12} \\ \varepsilon_{\max }=14400\end{array}\right|$ Diphényle-cétène............ $\mid \begin{aligned} & \nu_{\max }=1154.1012 \\ & \Sigma_{\max }=17800\end{aligned}$.

De ces observations nous pouvons conclure que les diazoïques ont leur premier groupe chromophore moins fortement attaché au reste de la molécule que les cétènes. Les cétènes absorbant plus que les diazoïques ont une plus grande activité chimique, ils réagiront plus vite avec d'autres combinaisons. Toutefois les deux effets de la non-saturation et de la labilité se combinant, la différence est difficile à observer. Aux réactions qui ne dépendent que de la force de liaison appartient la décomposition des diazoïques en $\mathrm{N}_{2}$ et en radical méthylènique,

$$
\mathrm{R}_{2} \mathrm{C}=\mathrm{N} \equiv \mathrm{N} \quad \longrightarrow \quad \mathrm{R}_{2} \mathrm{C}=+\mathrm{N}_{2} \quad \longrightarrow \quad \mathrm{R}_{2} \mathrm{C}=\mathrm{CR}_{2}
$$

et celle des cétènes en oxyde de carbone et radical méthylènique :

$$
\mathrm{R}_{2} \mathrm{C}=\mathrm{C}=\mathrm{O} \longrightarrow \mathrm{R}_{2} \mathrm{C}=+\mathrm{C}=\mathrm{O} \quad \longrightarrow \quad \mathrm{R}_{2} \mathrm{C}=\mathrm{CR}_{2}
$$

La décomposition des diazoïques se produit en général déjà à leur température d'ébullition à pression normale. Staudinger et Endle $\left({ }^{1}\right)$, ont déterminé la température de décomposition du diphén ylecétène et du diméthylecétène. Ils ont observé la formation tétraphényle-éthylène et de tétraméthyle-éthylène entre $650^{\circ} \mathrm{C}$ et $700^{\circ} \mathrm{C}$. On voit qu'ils se décomposent à plus haute température que les diazoïques. Ceci est en accord avec l'hypothèse que nous avons déduite du déplacement des bandes d'absorption.

D'autre part l'atome d'azote terminal d'un diazoïque est moins saturé que l'atome d'oxygène des cétènes. Tous deux peuvent additionner de la triphénylophosphine; mais le produit formé avec le cétène est le moins stable des deux.

$$
\begin{aligned}
& \left.\mathrm{R}_{2} \mathrm{C}=\mathrm{N} \equiv \mathrm{N}+\mathrm{PR}_{3} \quad \longleftrightarrow \quad \mathrm{R}_{2} \mathrm{C}=\mathrm{N}-\mathrm{N}=\mathrm{PR}_{3} \quad \text { stable ( }{ }^{2}\right)
\end{aligned}
$$

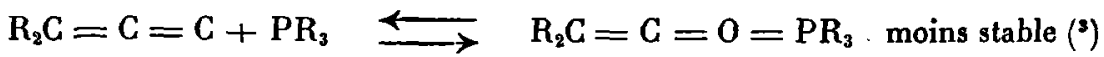

(1) H. Staudinger et Endie, B., $46 ; 1437$ (1913). 861 (1921).

(2) H. Staudinger et J. Meyer, Helo., 2, 636 (1919); Staudinger et Hauser, Helv., 4

(') H. Staudinger et J. Meyer, Helv., 2, 612 (1919). 
Les réactions qui dépendent de la labilité même des chromophores sont pour les diazoïques comme pour les cétènes celles qui ont lieu avec des combinaisons possédant un atome d'hydrogène mobile, alcools, amines primaires et secondaires.

Ces réactions vu l'instabilité du groupe $=\mathbf{N} \equiv \mathbf{N}$ chez les diazoïques sont très complexes et n'ont pas été étudiées en détail ( ${ }^{1}$ ). Pour les cétènes nous possédons des données plus précises; nous l'exposons dans le chapitre suivant.

L'analogie entre le chromophore $=\mathrm{N} \equiv \mathrm{N}$ et $=\mathrm{G}=0$ est très grande. ll est intéressant de noter que la somme des poids atomiques de ces deux radicaux est égale,

$$
\rangle_{2}=28 \quad>\mathrm{C}=0=28
$$

ainsi que la somme de leur " nombre atomique ",

$$
\mathrm{N}_{2}=14 \quad \mathrm{C}+\mathrm{O}=14
$$

La somme des charges nucléennes et le nombre des électrons libres sont les mêmes. D'après les théories modernes de G. $\mathrm{N}$. Lewis et Langmuir on représente ces radicaux par les mêmes symboles électroniques ( $\left.{ }^{2}\right)$.

La seule différence réside dans la répartition des charges c'est-à-dire la nature des atomes.

Une même analogie chimique existe entre les cétènes et les isocyanates. Spectrographiquement ils semblent différer. Les isocyanates ne possèdent pas de fonction carbonyle; ils ne possèdent pas la bande qui correspond à celle des cétènes dans le visible.

Dans l'extrême ultra-violet ils présentent une forte absorption mais beaucoup

\begin{tabular}{|c|c|c|}
\hline Substituant & Cétènes & Isocyanates \\
\hline Ethyle (Dióthylecétène)............ & $\left|\begin{array}{l}v_{\max }=1338.10^{12} \\
\varepsilon_{c_{\max }}=361\end{array}\right|$ & (isocyanate d'éthylę) $\begin{aligned} \nu_{\max } & =1473.1012 \\
\varepsilon_{i_{\max }} & =111 ; \frac{\varepsilon_{c}}{\varepsilon_{i}}=3.3\end{aligned}$ \\
\hline Phényle (Diphénylecétène) ........... & $\begin{array}{l}v_{\max }=1154.10^{12} \\
\varepsilon_{c_{\max }}=17800\end{array}$ & $\begin{aligned} \text { (isocyanate de phényle) }{ }_{\max }=1323.1012 \\
\varepsilon_{i_{\max }}=13550 ; \frac{\varepsilon_{c}}{\varepsilon_{i}}=1,3\end{aligned}$ \\
\hline
\end{tabular}
moins intense que celle des cétènes :

En rapportant l'intensité d'absorption des isocyanates à celle de l'urée, qui est exceptionnellement faible $\left({ }^{3}\right)$, nous pouvons écrire que les :

$$
\frac{\text { Céténes }}{\text { Cétones }} \sim \frac{\text { Isocyanates }}{\text { Uréides }} \text {. }
$$

(1) Staudinger, Hels., V, 91 (1922).

(2) Journal of American Chem. Soc., 1918 à 1920.

(3) Victon Henri, Etudes de Photochimie, p. 202. 
La labilité des isocyanates est très petite; leurs réactions avec les amines ou les alcools doivent être beaucoup plus lentes que celles des cétènes.

Comme pour les amides et les éthers-sels, la fonction carbonyle n'existe pas chez les isocyanates ou du moins n'est pas apparente ( $\left.{ }^{1}\right)$.

Le second chromophore, $-\mathrm{N}=\mathrm{C}=$ ne subit pas son action et sa stabilité est plus grande que celle de la liaison éthylénique des cétènes. Ceci apparaît très nettement dans les spectres, dans la valeur du rapport des intensités $\left(\frac{\varepsilon_{c}}{\varepsilon_{i}}\right)$.

Pas plus dans les cétènes que dans les isocyanates le groupe carbonyle n'avait pu être caractérisé par des méthodes chimiques. Il suffit de jeter un coup d'œil sur le spectre de l'un et de l'autre pour s'assurer de sa présence dans les cétènes et de son absence chez les isocyanates (voir partie expérimentale pages 77-79).

Entre les diazoïques et les azides la relation est analogue. Pour les azides, l'action du premier chromophore apparaît encore mais est très affaiblie. Sa bande est moins déplacée vers le rouge que pour les diazoïques l'effet du second chromophore est donc atténué :

\begin{tabular}{|c|c|c|}
\hline Substituant & Diazoïques & Azides \\
\hline 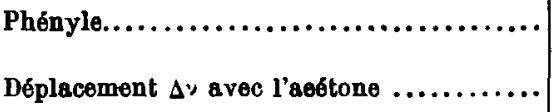 & $\begin{array}{l}v_{\max }=600.10^{12} \\
\varepsilon_{\max }=89 \\
509.10^{12}\end{array}$ & $\begin{array}{l}\nu_{\max }=1005 \\
\varepsilon_{\max }=132 \\
\\
104.10^{12}\end{array}$ \\
\hline
\end{tabular}

Spectrographiquement et chimiquement diazoïques et azides sont semblables. La force de liaison qui lie le chromophore $=\mathrm{N} \equiv \mathrm{N}$ à la molécule est plus grande pour ces derniers.

Il est intéressant de remarquer que l'effet du groupe imide $\mathbf{R}-\mathbf{N}=$ est de déplacer la bande de $=\mathrm{N} \equiv \mathrm{N}$ vers l'ultra-violet; la différence de fréquence pour les dérives phénylés est $\Delta \nu=\mathbf{4 0 5 . 1 0 ^ { 1 2 }}$.

Si à la fréquence du diphénylecètène on ajoute ce $\Delta v$ on devrait obtenir la fréquence de la bande du carbonyle pour les isocyanates.

$$
\begin{aligned}
& \vee_{\max } D_{p} \text { cétène }=748.10^{12} \\
& \quad 748.10^{12}+405.10^{12}=1153.10^{12}
\end{aligned}
$$

On obtient la fréquence $1153.10^{22}$.

Pour celle-ci l'isocyanate de phényle possède déjà un coefticient d'absorption $\varepsilon=200$ et la bande du $\mathrm{G}=0$ serait complètement recouverte.

On peut conclure de cela que la fonction carbonyle n'est pas inexistante pour

(1) Staudinger et Con, par leur mesure de vitesses de réactions du diphénylecétène avec des combinaisons carbonylées, ont aussi observé cette absence d'activité chimique du groupe $=\mathrm{C}=\mathrm{O}$ chez les amides et les éthers-sels. Ann., $384_{2}$ p. 38 (1911). 
les isocyanates, comme nous l'avons écrit plus haut, mais que la présence du groupe imide, $\mathbf{R}-\mathbf{N}=$, en absorbe l'effet.

Entre les isocyanates et les azides il existe aussi une grande similitude. Tous deux possèdent dans le spectre de leur dérivé phénylé des bandes aromatiques.

\begin{tabular}{|c|c|c|c|c|c|c|}
\hline \multirow{2}{*}{ Substituant } & \multicolumn{4}{|c|}{ Isocyanater } & \multicolumn{2}{|c|}{ Azides } \\
\hline & 1 & & & & $v_{\operatorname{mas}}=1005.1012$ & $\varepsilon_{\max }=132$ \\
\hline \multirow[t]{2}{*}{ Phényle.................. } & $\begin{array}{l}a^{\prime} \\
a^{\prime \prime} \\
a^{\prime \prime \prime}\end{array}$ & $\begin{array}{c}v_{\max }=1082.1012 \\
\quad=1111.1012 \\
\quad=1129.10+2\end{array}$ & $\begin{aligned} \varepsilon_{\max } & = \\
* & = \\
= & =\end{aligned}$ & $\begin{array}{l}440 \\
350 \\
275\end{array}$ & $\begin{aligned} v_{\max } & =1055.1012 \\
& =1080.1012 \\
& =\ldots \ldots \ldots . .\end{aligned}$ & $\begin{aligned} \varepsilon_{\max } & =2.450 \\
& =2750 \\
& =\ldots . .\end{aligned}$ \\
\hline & 2 & $\star=1323.1012$ & $x=15$ & 3550 & $\times=1215.10^{12}$ & $x=8900$ \\
\hline
\end{tabular}

Ces relations sont identiques à celles qui existent entre les diazoïques et les cétènes.

$$
\begin{gathered}
v_{\max } \text { cétènes }-v_{\max } \text { diazoïques }=1154.10^{12}-1057.10^{12}=97.10^{12}=\Delta v \\
v_{\max } \text { isocyanates }-v_{\max } \text { azides }=1323.10^{12}-1215,10^{12}=108.10^{12}=\Delta v \\
\frac{\varepsilon_{\mathrm{II}} \text { cétènes }}{\varepsilon_{\mathrm{II}} \text { diazoïques }}=1,24 \quad \frac{\varepsilon_{\mathrm{II}} \text { isocyanates }}{\varepsilon_{\mathrm{II}} \text { azides }}=1,52
\end{gathered}
$$

Nous en concluons que :

$$
\frac{\text { les diazoïques }}{\text { les azides }} \sim \frac{\text { les cétènes }}{\text { les isocyanates }}
$$

Les relations que nous avons pu établir entre ces quatre classes de combinaisons, en considérant les spectres ultra-violets, peuvent aussi être déduites des spectres infra-rouges.

$M^{110} P$. Honnegger ( $\left.{ }^{1}\right)$, a mesuré l'absorption infra-rouge de quelques-uns de nos dérivés, au laboratoire de M. Victor Henri.

Nous donnons dans une table les longueurs d'ondes et les fréquences qu'elle a observé :

(1) Victor Henri, Etudes de Photochimie, 1919, p. 64. 


\begin{tabular}{|c|c|c|c|c|c|c|c|c|}
\hline \multicolumn{3}{|c|}{$\underset{\mathrm{CH}_{3} \mathrm{COCH}_{3}}{\text { Acétone }}$} & \multicolumn{2}{|c|}{$\mathbf{R}_{2} \stackrel{\text { Cétènes }}{=} \mathbf{C}=\mathrm{CO}$} & \multicolumn{2}{|c|}{$\begin{array}{l}\text { Acétate d'éthyle } \\
\text { diazoïque } \\
\mathrm{N}_{2} \mathrm{CKCOOC}_{2} \mathrm{H}_{5}\end{array}$} & \multicolumn{2}{|c|}{$\begin{array}{l}\text { Azide d'acétate d'éthyle } \\
\mathrm{N}_{3} \mathrm{CH}_{2} \mathrm{COOC}_{2} \mathrm{H}_{5}\end{array}$} \\
\hline & $\lambda_{\max }$ & $v_{\max }$ & $\lambda_{\max }$ & $v_{\max }$ & $\lambda_{\max }$ & $v_{\max }$ & $\lambda_{\max }$ & $v_{\max }$ \\
\hline I. . . . . . . . & $3,3 \mu$ & $92.10^{12}$ & $3,5 \mu$ & $85.7 .10^{12}$ & $3,4^{\prime} \mu$ & $88,2,10^{12}$ & $3,4 \mu$ & $88,2.10^{12}$ \\
\hline II $\ldots \ldots \ldots \ldots$ & $4,9 \mu$ & $61.10^{18}$ & $4,7 \mu$ & $69,8.10^{1 z}$ & $4,7 p$ & $63,8.1012$ & $4,7 \mu$ & $63,8.1012$ \\
\hline III $\ldots . .$. & $5,7 \mu$ & $52,6.10^{12}$ & $5,7 \mu$ & $52,710^{12}$ & $5,8 \mu$ & $51,7.10^{12}$ & $5,8 \mu$ & $51,7.10^{12}$ \\
\hline
\end{tabular}

\begin{tabular}{|c|c|c|}
\hline & $\begin{array}{r}\text { Isocyanate } \\
\text { et Carboiimide }\end{array}$ & $\begin{array}{l}=\mathrm{C}=\mathrm{O} \\
=\mathrm{C}=\mathrm{N}-\mathrm{R}\end{array}$ \\
\hline$\cdot$ & $\lambda_{\max }$ & $v_{\max }$ \\
\hline $1 . \ldots \ldots \ldots \ldots \ldots \ldots$ & $3,5 \mu$ & $85,7.1012$ \\
\hline II $\ldots \ldots \ldots \ldots \ldots \ldots$ & $4,6 \mu$ & $65.2 .10^{12}$ \\
\hline III $\ldots \ldots \ldots \ldots \ldots \ldots$ & $5,7 \mu$ & $52,7.10^{12}$ \\
\hline
\end{tabular}

On voit que leurs bandes caractéristiques correspondent entre elles et à celles. de l'acétone. La bande I se retrouve aussi pour les hydrocarbures. Par contre les. bandes II et III sont des bandes caractéristiques de l'état de non-saturation de la molécule ( ${ }^{1}$ ), ll existe pour nos dérivés certaines différences d'intensité, d'où $M^{110}$ Honnegger tire des conclusions identiques aux nôtres.

(1) Theise, Zurich, 1922 (du laboratoire de V. HexaI). 


\section{CHAPITRE II}

\section{ETUDE SP ECIALE DES SPECTRES D'ABSORPTION DES C ÉTËNES}

Le spectre des cétènes est caractéristique. Il se compose de deux bandes : l'une dans l'ultra-violet extrême esı très intense ; l'autre qui possède une grande analogie avec celle de l'acétone, apparait à la limite du spectre visible. La position et l'intensité de chacune d'elles dépend du substituant du radical méthylènique.

Tableau donnant les positions des bandes et les valeurs du coefficiènt moléculaire d'absorption pour les maxima des cétènes.

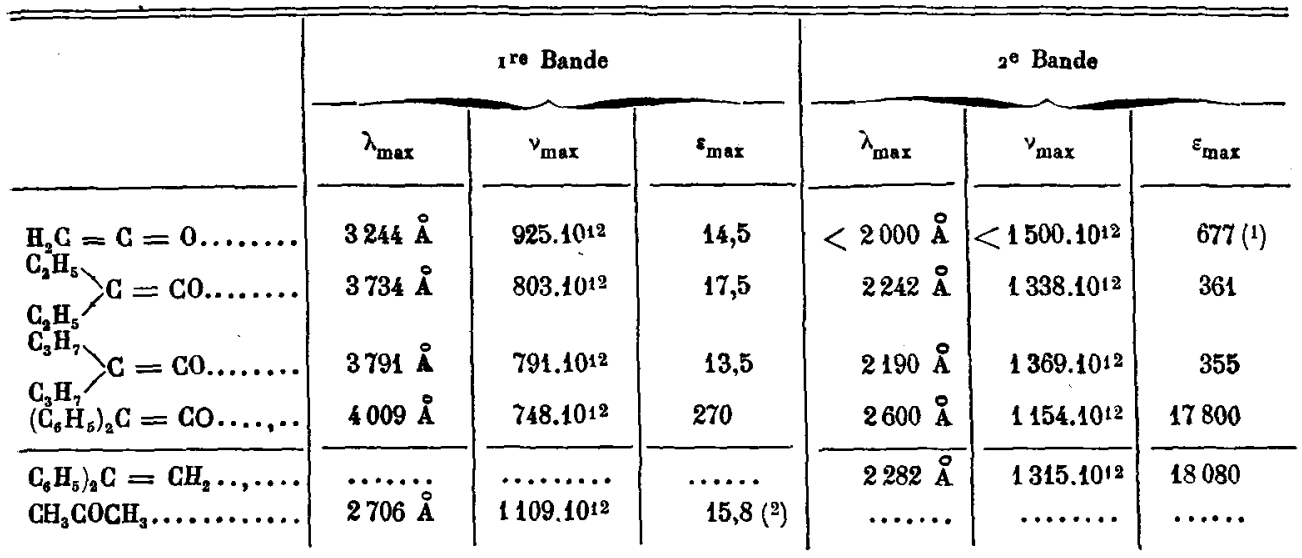

(1) Nous avons étudié les spectres entre les $\lambda=5000 \AA$ et $\lambda=2000 \AA$, soit les fréquences $v=600.10^{12}$ et $v=1500.10^{12}$.

(2) Nous empruntons les valeurs pour l'acétone à Victor Henri, Etudes de Photochimie, 1919 , p. 70 . 
Des lois de répartition des bandes de Victor Henri, (voir page 3) nous pouvons déduire que le déplacement vers le visible d'une bande propre à un chromophore est proportionnel à l'action d'un second chromophore ( $\left.{ }^{1}\right)$.

La variation de l:intensité dépend de la labilité même du chromophore.

Dans les spectres des cétènes nous observons les deux effets. Voyons d'abord ce que nous pouvons conclure du déplacement de la bande vers le visible. Il peut être fonction de la masse du substituant, $\left({ }^{2}\right)$, et de sa nature chimique. Nous rapportons donc le déplacement observé à la masse; nous calculons le déplacement spécifique.

\begin{tabular}{|c|c|c|c|}
\hline Substituant & $\begin{array}{l}\text { Poids moléculaire } \\
\text { de colvi-ci }\end{array}$ & $\begin{array}{c}\text { Déplacement observé } \\
\text { par rapport xu cétène } \\
\Delta y \text {. }\end{array}$ & $\begin{array}{c}\text { Déplacement spéci ique } \\
\mathscr{D}_{y}^{\text {cétènes }}\end{array}$ \\
\hline 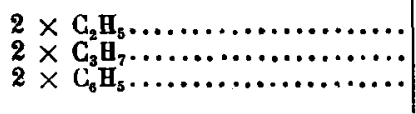 & $\begin{array}{r}58 \\
86 \\
154\end{array}$ & $\begin{array}{l}-122.1012 \\
=134.1012 \\
=177.10^{12}\end{array}$ & $\begin{array}{l}-21,0.1011 \\
-15,6.1011 \\
-11,5.1011\end{array}$ \\
\hline
\end{tabular}

Comme valeur de comparaison pour les déplacements spécifiques trouvés, nous devons prendre le déplacement entre le diéthylecétène et le dipropylecétène. Ces deux corps sont substitués par deux radicaux de méme nature ; toute différence dans leurs propriétés est due à la différence des masses.

Le déplacement entre le diêthyle et le dipropyle-cétène est égal à $\nu=-12.10^{12}$; il se rapporte à deux groupes méthylènes $=\mathrm{CH}^{2}$. Ceux $\cdot c i$ ont un poids moléculaire de 2 fois 14 soit 28 . Le déplacement spécitique normal est

$$
\frac{12.10^{12}}{28}=4,310^{11}=D_{\text {Norm. }}^{\text {cótène }}
$$

L'exaltation du déplacement spécifique pour les cétènes est le rapport de leur déplacement spécifique propre au déplacement spécifique normal.

Elle est poer :

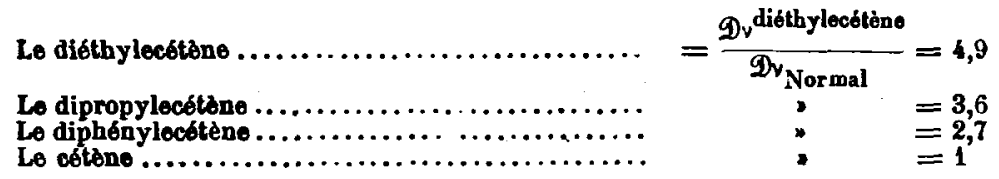

Pour les cétènes l'exaltation du déplacement spécifique n'est autre chose que le coefficient par lequel il faut multiplier la valeur qui représenterait la non-saturation ou l'instabilité du cétène, $\mathrm{H}^{2} \mathrm{G}=\mathrm{G}=0$, pour obtenir celle de ses dérivés.

(1) Staudinger et Con, Ann., 384, p. 59 (1911).

(2) Calcul des bandes d'absorption pour des corps de constitution connue. Etudes de Photochimie, p. 196.

Guillaume C. Lardy. 
Soit $k$ cette valeur inconnue, la non-saturation des cétènes substitués serait en première approximation :

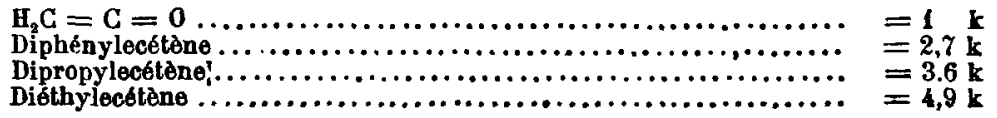

Ces valeurs s'ordonnent comme le devraient les vitesses de polymérisation des divers cétènes dans des états correspondants. Nous donnons ici les températures correspondantes pour les différents cétènes. Ce sont celles des points d'ébullitions sous une pression de 10 à $15 \mathrm{~mm}$ de mercure $\left({ }^{1}\right)$.

\begin{tabular}{|c|c|c|}
\hline & T. absolue & T. centigrades \\
\hline 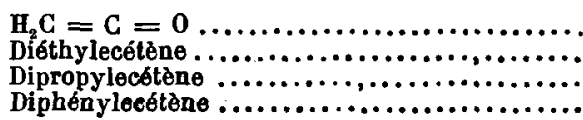 & $\begin{array}{l}175^{\circ} \\
292^{\circ} \\
308^{\circ} \\
423^{\circ}\end{array}$ & $\begin{array}{r}98^{\circ} \mathrm{C} \\
+\quad 190 \mathrm{C} \\
35^{\circ} \mathrm{C} \\
150^{\circ} \mathrm{C}\end{array}$ \\
\hline
\end{tabular}

Les mesures qui ont été faites de la vitesse de polymérisation ne nous fournissent pas de preuves assez exacte pour vèrifier notre hypothèse $\left({ }^{2}\right)$. Qualitativement ce qu'on connaît de la stabilité des cétènes semble être conforme à la série précédente.

Quant à la labilité des cétènes, c'est-à-dire la vitesse avec laquelle ils réagissent avec d'autres combinaisons chimiques, elle est due à l'activité du groupe carbonyle lui-même. Elle nous est représentée par l'intensité d'absorption de la $1^{\text {re }}$ bande. Pour le cétène et ses dérivés aliphatiques elle est à peu près égale à celle du $=\mathrm{C}=\mathrm{O}$ dans l'acétone.

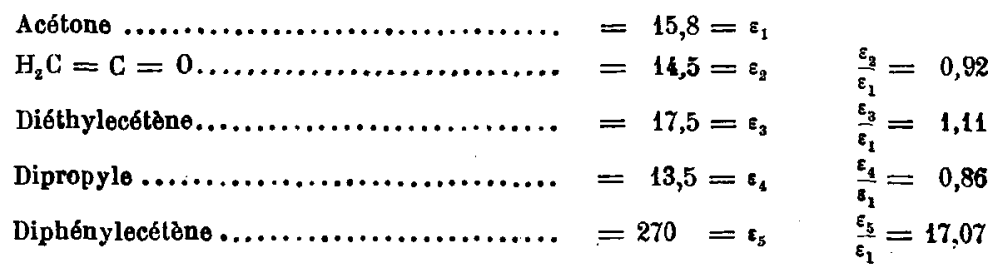

Pour le diphénylecétène elle est beaucoup plus forte et les réactions qui en dépendent doivent avoir une vitesse peut-être vingt fois plus grande que celle des autres.

La réaction des cétènes avec les alcools en est un exemple.

La présence de deux chromophores dans les cétènes, de deux centres de labilité, donne deux possibilités de réaction :

l'une avec le groupe $=\mathrm{G}=\mathrm{C}=$ réactions du premier ordre l'autre avec le groupe $=\mathrm{C}=0$ réactions du second ordre $\left({ }^{3}\right)$

(1) Guldaerg, Zeit. Phys. Chem., 5 ; 374 (1890) et Guye, Bull. (3), 4; 262 (1890).

(2) Staudinger et Strong, Helv., VI, p. 291 (1923).

(3) Staudinger, Hel\%., V, p. 97 (1922). 
Dans les réactions du premier ordre se trouve la polymérisation. Elle dépend de la non-saturation du groupe éthylène, $=\mathrm{C}=\mathrm{C}=$ elle est la plus rapide pour les cétènes dont l'exaltation du déplacement est la plus grande, soit pour les céthènes aliphatiques. Ceci est conforme à l'expérience. Staudinger et Strong, ( $\left.{ }^{2}\right)$, ont mesuré les vitesses de polymérisation de plusieurs céténes. Ils ont trouvés que le diéthyle-cétène polymérisait plus vite que dipropyle.

Pour le diphényle-cétène la vitesse de polymérisation, dont le produit serait le tétra-phényle-dicétocyclobutane, est petite. Staudinger et ses collaborateurs ne l'ont obtenu qu'en chauffant le diphénylecétène en présence d'un catalyseur $\left({ }^{2}\right)$. (La réaction d'addition à des groupes carbonyles, comme la résine que forme le diphényle-cétène lorsqu'on le chauffe quelques temps à $160^{\circ} \mathrm{C}$., sont très probablement des réactions dúes à l'activité spéciale du carbone du groupe $=\mathrm{C}=\mathrm{O}$ ).

Pour le cétène, $\mathrm{H}^{*} \mathrm{C}=\mathrm{C}=\mathrm{O}$, nous ne pouvons rien dire. A température normale sous pression, il peut polymériser instantanément avec fort dégagement de chaleur $\left({ }^{3}\right)$. Comme aucun des autres cétènes n'a été maintenu dans de semblables conditions $\left(\grave{a}+210^{\circ} \mathrm{C}\right.$ pour le diéthyle-cétène et $+427^{\circ} \mathrm{C}$. pour le diphénylecétène) nous n'avons pas de comparaison possible.

Pour les réactions du second ordre nous avons des exemples dans l'addition d'alcools, d'amines primaires ou secondaires, avec les cétènes ( ${ }^{*}$ ).

D'après l'intensité de la bande carbonylique, le diphénylecétène doit réagir très vivement avec l'eau, l'alcool et les bases. Les cétènes aliphatiques et le cétène par contre beaucoup plus lentement. (peut-être 20 fois).

Aucune vitesse de réaction entre les cétènes aliphatiques et l'alcool n'a pu être mesurée. La lenteur de leur réaction est telle que les résultats sont faussés ( ${ }^{5}$ ).

Avec le diphénylecétène la réaction est rapide, ce qui est en accord avec le résultat prévu de l'intensité de la $1^{\text {re }}$ bande. Nous avons pu faire de nombreux essais (2) et avons déterminé avec exactitude que la réaction est bimoléculaire. Le cétène agit lui-méme comme catalyseur en sorte que la loi d'action des masses n'est pas directement applicable.

Nous avons déduit l'ordre de la réaction catalytique du cétène par la méthode différentielle de van t'Hoff.

(1) Helv., VI, 296 (1923).

(ग) B., 47, p. 40-48 (1914), et Ann., 384, p. 110 (1911).

(अ) Staudinger, Les Cétènes, p. 137.

(4) Staudinger et P. Meyer sont parvenus à isoler des dérivés de l'acide méthylène carbonique (Helv., V, p. 656 (1922).

$$
\mathrm{R}, \mathrm{C}=\mathrm{C}=\mathrm{O}+\mathrm{C}_{2} \mathrm{H}_{6} \mathrm{OH} \quad \longrightarrow \quad \mathrm{R}_{2} \mathrm{C}=\mathrm{C}_{\mathrm{OH}}^{-\mathrm{OC}_{2} \mathrm{H}_{5}} \quad \longrightarrow \quad \mathrm{R}_{2} \mathrm{CHCOOC}_{2} \mathrm{H}_{5}
$$

(5) Pour suivre une réaction des cétènes, on abandonne plusieurs échantillons en flacons fermés d'une solution de cétène et d'alcool dans l'éther (la concentration étant connue) dans un thermostat. Après un temps déterminé on retire l'un des flacons, ajoute à la solution de l'éther humide et agite jusqu'à décoloration complète, ce qui a lieu en quelques secondes pour le diphénylecétène. Il se forme par addition d'eau une quantité d'acide équivalente à celle du cétène n'ayant pas réagi, qu'on tître avec de la lessive de soude ou de la lessive de baryte. (SutEan Thèse, Zurich, 1920, laboratoire de M. Staudinger). 
H est intéressant de noter que les réactions du premier ordre, comme toutes celles qui donnent naissance à un cycle de quatre carbones, sont catalysées par le produit de réaction. Elles sont indifférentes à la présence d'une base tertiaire $\left.{ }^{(}\right)$.

Les réactions du second ordre sont catalysées par la présence de quinoléine même en très petite quantité ${ }^{2}$ ).

(1) M. Capato a étudié la réaction du diphényle-cétène avec le styrolène. Il a pu déterminer l'action catalytique du produit de réaction. Cette réaction obéit à la loi d'action des masses transformée par Ostwald pour les cas d'autocatalyse :

$$
\frac{d x}{d t}=h_{1}(a-x)^{2}+h_{2} x(a-x)^{2}
$$

Il a trouvé pour $k_{\mathrm{z}}$ et $k_{\mathrm{z}}$ des valeurs constantes pour $20^{\circ} \mathrm{C}$ et $40^{\circ} \mathrm{C}$.

(2) D'après les essais de M. Richard :

Diphénylecétène + alcool éthylique à $25^{\circ}$

ont réagi après une heure san's catalyseur ...................

ont réagi après une heure avec 0,0001 de quinoléíne.............

ont réagi après une heure avec 0,0013 de quinoléíne.............. $82,7 \%$ 


\section{CHAPITRE III}

\section{SUR LA NATURE DES DIMERES ET DES CETENGS}

Les dimères des cétènes sont isomères avec certains corps synthétisés, à partir de l'éther sel du diacide de l'acétone $\left(^{(2}\right)$. Cette question a soulevé une discussion de grand intérêt. Car cette isomérie a deux causes possibles : soit une simple desmotropie (formule I et II) soit une " isomérie de valences". (formule III).

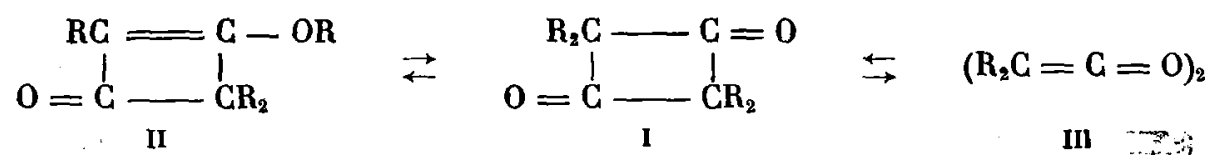

Schroeter a soutenu celte dernière hypothèse ( $\left.{ }^{2}\right)$. Il supposait qu'un dimère de cétène était un agrégat sans structure définie. Son argument principal était la différence de la décomposition pyrogénée des dimères des cétènes et de ses produits synthétisés.

Par exemple le dimère de l'éther éthylique du mono-acide de l'éthylecétène :<smiles>CCOC(=O)C1(CC)CC(=O)C(CC)(C(=O)OCC)C1=O</smiles>

se sépare en deux molécules de cétène en distillant dans le vide sous une pression de 15 millimètres de mercure.

(1) Schroeter, B., 49, 2697, (1916).

\section{HOOC.CH$\left(\mathrm{CH}_{3}\right) \cdot \mathrm{CO} \cdot \mathrm{CH}\left(\mathrm{CH}_{2}\right) \mathrm{COOH}$}

(9) Schroeter $B_{.2} 4922697$ (1916), et Zeit. Angew. Chem., 24, 292 (1911). 
De même pour le dérivé monométhylé :<smiles>CCOC(=O)C1(C)C(=O)C(C)(OCC)C1=O</smiles>

qui se décompose dans les mémes conditions. Nous n'avons toutetois pas pu isoler le produit monomoléculaire $\left(^{(}\right)$.

Le corps obtenu par synthèse par Schroeter (loc. cit).<smiles>CCOC(=O)OC1(C)C2(C)C(=O)C1(C)C2=O</smiles>

distille saus se décomposer aussi bien dans un vide de $15 \mathrm{~mm}$ de mercure que dans le vide absolu.

Cette - isomérie de valences * eût été pour la chimie organique d'une importance capitale. Car elle aurait montré l'existence de deux sortes de valences ou mieux de deux sortes d'affinités, l'une liant les atomes entre eux, l'autre liant les molécules. Nous avons déjà exposé dans la préface notre opinion sur ce sujet. Nous avons insisté sur le fait que l'intensité d'une liaison peut varier selon la constitution d'un corps, mais qu'une différence essentielle, entre combinaisons moléculaires et combinaisons atomiques, ne nous semblait pas pouvoir exister.

Staudinger $\left({ }^{2}\right)$ conserva malgré les dires de Schroeter sa conception d'une isomérie tautomérique. Il trouvait dans l'instabilité d'un cycle formé de quatre atomes de carbones ainsi que par l'accumulation de groupes carbonyles des raisons: suffisantes pour expliquer une décomposition facile à la chaleur. Quant à la diftérence entre les produits de décomposition des combinaisons de Schroeter et des dimères des cétènes il la supposait due à la présence d'une liaison éthylènique dans le a carré " du a cyclobutenolone ". Les faits lui ont donné raison.

De nouvelles expériences entreprises par deux élèves de Staudinger, Paul Meyer $\left({ }^{3}\right)$ et Alfred Rheiner (") ont démontré que la stabilité des dérivés du cyclobutane est influencée par une substitution. L'introduction d'un groupe phényle par exemple l'amoinúrit fortement.

Dernièrement Dieckmann et Wittmann ( ${ }^{5}$ ) ont prouvé que par la réaction de Schroeter on obtient, non pas un dérivé du dicétocyclobutane (I), mais de son tautomère le a cyclobutenolone (II).

(1) D'après les essais de MM. Tripet et Gonset.

(2) $B ., 53$, p. 1073 et 1085 (1920).

(9) Thèse (Zurich, 1922, du laboratoire de M. Staudivarn).

(4) Thèse (Zurich, 1923, du laboratoire de M. Staudinger).

(5) $B$., $55 ; 3331$ (1922). 
Dans l'idée que la connaissance des propriétés physiques de ces dicétones pouvait amener quelque clarté, nous avons étudié les spectres d'absorption des dimères des cétènes.

Si les dimères des cétènes sont des combinaisons moléculaires, nous ne pouvons nous attendre à y trouver des fonctions chimiques bien définies. Par contre la présence d'une fonction carbonyle, ou mieux des variations de cette fonction identiques à celles qu'on observe chez les $\beta$-dicétones, serait un argument probant en faveur des conceptions de Staudinger.

Les résultats de notre analyse spectrale sont dans ce sens et nous pouvons affirmer que l'hypothèse de Schroeter sur la nature des dimères des cétènes n'est pas fondée.

Ceux-ci sont des dérivés des $\beta$-dicétones. Toutefois les observations que nous avons faites montrent que les hypothèses actuelles ne sont pas tout à fait conformes à la réalité, tout spécialement en ce qui concerne les diméres des aldocétènes.

1. Spectres d'absorption des dicétones. - Les spectres d'absorption des combinaisons carbonylées possèdent une bande ultraviolette pour

$$
\lambda_{\max }=2706 \AA \quad v_{\max }=1109.10^{12} \quad \varepsilon_{\max }=15,8
$$

L'introduction d'un second carbonyle a les effets énoncés par Victor Henri dans la loi sur la position des bandes. (voir page 3).

Pour les $\alpha$-dicétones nous aurons en conséquence une bande de même intensité que celle de l'acétone à la lisière du spectre visible. déplacée.

Pour les $\beta$-dicétones non-énolisables la bande ne sera pas aussi fortement

Dans les deux cas la bande large de l'acétone se décompose en 3 ou 4 bandes étroites dont l'intensité décroit avec la fréquence. (voir loi de la structure des bandes spectrales de Victor Henri, page 4 ).

a) En effet pour les $\alpha$-dicétones, le diacétyle, le déplacement vers le rouge de la bande cétonique est :

$$
\Delta v=v_{\max } \text { acélone } 1109.10^{12}-v_{\max } \text { diacétyle } 703.10^{12}=40610^{12}
$$

et la bande se décompose en quatre bandes fines pour : ( $\left.{ }^{1}\right)$

$$
\begin{aligned}
& \lambda_{1_{\max }}=4494 \AA \quad v_{1}=667.10^{\prime 2} \quad \varepsilon_{1}=20,5 \quad \varepsilon \text { acétone }=15,8 \\
& \lambda_{2} \prime=4434 " \quad v_{2}=676 " \quad \varepsilon_{2}=18,8 \\
& \left.\lambda_{3}\right)=4240 \text { v } \quad v_{3}=707 n \quad \varepsilon_{3}=19,5 \\
& \lambda_{4} "=4051 " \quad v_{4}=740 " \quad \varepsilon_{4}=15
\end{aligned}
$$

Le même effet avait été observé par Luthy pour le glyoxal, Klingstedt pour la paraquinone et Castille pour le benzile.

(2) Voir partie exérimentale, page 83 . 
b) ß-dicétones à caractère non-énolisable:

\begin{tabular}{|c|c|c|c|c|c|c|c|}
\hline \multirow{2}{*}{ Dicétocyclobutane } & \multicolumn{4}{|c|}{ I re Bande } & \multicolumn{3}{|c|}{$2^{\circ}$ Bande } \\
\hline & \multicolumn{2}{|c|}{$\lambda_{\max } \AA$} & $y_{\max }$ & $\varepsilon_{\max }$ & $\lambda_{\max } \AA$ & $\nu_{\text {max }}$ & $\varepsilon_{\max }$ \\
\hline Tótra-éthylé.... & $\begin{array}{l}1 \\
2 \\
3\end{array}$ & $\begin{array}{l}3444 \\
3142 \\
3013\end{array}$ & $\begin{array}{l}871.10^{12} \\
955 * \\
996\end{array}$ & $\begin{array}{r}8,7 \\
21,7 \\
16,5\end{array}$ & 2185 & $1373.10^{12}$ & 120 \\
\hline Tétrapropylé.......... & $\begin{array}{l}1 \\
2 \\
3\end{array}$ & $\begin{array}{l}3535 \\
3125 \\
3013\end{array}$ & $\begin{array}{l}848.10^{12} \\
960.10^{12} \\
996\end{array}$ & $\begin{array}{l}23,3 \\
50 \\
35,1\end{array}$ & 2388 & $1344.10^{12}$ & 180 \\
\hline Tétra-allylé........ & $\begin{array}{l}1 \\
2 \\
3\end{array}$ & $\begin{array}{l}3447 \\
3151 \\
3014\end{array}$ & $\begin{array}{l}872.10^{12} \\
952 \\
996\end{array}$ & $\begin{array}{l}17,5 \\
51,5 \\
40\end{array}$ & 2066 & $1452.10^{12}$ & 2711 \\
\hline Diméthyló-diphónyylé. & $\begin{array}{l}1 \\
2 \\
3 \\
4\end{array}$ & $\begin{array}{l}3470 \\
3180 \\
3015 \\
2934\end{array}$ & $\begin{array}{c}865.10^{12} \\
944 * \\
996 » \\
1023 "\end{array}$ & $\begin{array}{l}105 \\
145 \\
143 \\
130\end{array}$ & $<2000 \AA$ & $>1500$ & $>50000$ \\
\hline
\end{tabular}

Comme nous l'avons prévu le śpectre d'absorption des dicétocyclobutanes tétrasubstitués, obtenues par polymérisation des cétocétènes, présente une $1^{\text {ro }}$ bande complexe qui, dans son aspect général, est semblable à celle des dérivés du groupe $\mathrm{C}=0$. Son intensité moyenne est, pour les homologues inférieurs, égale à celle de l'acétone. Elle croît avec la masse et selon la nature des substituants. Elle est particulièrement intense pour le dérivé phénylè ( $\mathbf{1}$ ).

Cette première bande se décompose en plusieurs bandes dont la $1^{\text {ro }}$ est assez large et les autres plus étroites.

Le premier maximum désigné par (1) dans notre tableau peut-être comparé à la bande très faible que Victor-Henri a mesuré pour l'acétone vers

$$
\nu=850.10^{12} \quad \varepsilon=0,00026
$$

Son intensité est pour nos produits beaucoup plus forte.

Les deux ou trois autres bandes étroites ont leur maximum moyen déplacé de $\nu=133,10^{12}$ par rapport à celui de l'acétone.

$$
\Delta v=\vee \text { acétone }-\vee \text { dicétone }=1109.10^{12}-976.10^{12}=133.10^{12}
$$

(ce sont les maxima que nous portons sous les numéros 2 , 3 et 4 dans la rubrique première bande de notre tableau).

Ces résultats forment dans leur ensemble une observation nouvelle. On ne

(1) Paul Meyer, loc, cit. 
connaissait jusqu'à cette dernière année aucun composé aliphatique possédant des bandes étroites.

La deuxième bande large de nos $\beta$-dicétones aliphatiquement substituées se trouve dans l'ultra-violet extrême pour $\nu=1350.10^{12}$ environ.

L'intensité varie selon la substitution :

$$
\begin{aligned}
\text { dérivé tétra-éthylé } \varepsilon_{\max } & =120 \\
\text { dérivé tétra-propylé } \varepsilon_{\max } & =180
\end{aligned}
$$

elle est particulièrement forte pour le dérivé tétra-allylé où

$$
\varepsilon_{\max }=2711 \text {. }
$$

(L'absorption très intense du dérivé diméthylé-diphénylé tient à la présence du noyau aromatique).

Alors que tout le reste du spectre du dérivé tétra-allylé et du tétrapropylé est très semblable, l'eftet des quatre liaisons éthyléniques se fait sentir sur cette dernière bande.

Cette exaltation de l'absorption causée par une double liaison a déjà été observée par Victor Henri $\left.{ }^{\boldsymbol{1}}\right)$.

Il calcule pour l'alcool propylique et l'alcool allylique un coefficient d'exaltation égal à 2,67 par double liaison. Pour les deux dicétocyclobutanes, nous obtenons une valeur égale à 3,76 .

Liaison éthylénique, groupe aromatique et $\beta$-dicétones cycliques se distinguent par une très forte absorption dans l'ultra-violet extrême. Ceci est en relation avec la théorie de Bayer sur la labilité des différents genres de liaisons entre les atomes de carbone.

2. $\beta$-dicétones à caractère énolisable. - L'acétylacétate d'éthyle et l'acétylacétone comme les autres combinaisons desmotropes possèdent un spectre très particulier. Leur forme énolique composée d'un groupe carbonyle et d'une double liaison hydroxylée en position conjuguée absorbe d'une façon très intense.

A concentration d'énol constante, le maximum peut se trouver à des fréquences différentes suivant la substitution et le solvant. L'intensité est proportionnelle à la concentration d'énol. Cette relation a été contrólée expérimentalement par Victor Henri et Grossmann $\left({ }^{2}\right)$, qui ont comparé l'intensité des spectres aux proportions d'énol déterminées par titration au brome.

Leurs résultats concordent et l'on peut dire que pour une même classe de corps le rapport entre les quantités d'énol pour chaque dérivé est donné par le rapport de l'intensité de la bande énolique.

Nous rapportons toutes nos observations à celles qui ont été faites pour

(1) Etudes de Photochimie, p. 103.

(2) Thèse (Zurich, 1923). 
l'acétylacétone. L'acétylacétate d'éthyle n'est pas comparable à nos dérivés du dicétocyclobutane car il ne possède pas deux carbonyles mais un carbonyle et un carboxyle.

L'acétylacétone possède une bande :

En solution hexanique $(92 \%$ de forme énol $)\left({ }^{1}\right)$.

$$
\lambda=2690 \AA \quad \varepsilon_{\max }=12100 \quad v_{\max }=1115.10^{12}
$$

En solution aqueuse alcaline

$$
\lambda=3000 \AA \quad \varepsilon_{\max }=14100 \quad v_{\max }=1000.10^{12}
$$

A ce groupe appartiennent les dérivés du dicétocyclobutàne dont on a pu isoler les deux formes cétonique et énolique.

Tel est le diphényle-dicétocyclobutane :

\begin{tabular}{|c|c|c|c|c|c|c|}
\hline \multicolumn{4}{|c|}{ a) dans l'hexane : } & \multicolumn{3}{|c|}{ b) dans l'alcool } \\
\hline , & $\lambda_{\max } \AA$ & $\nu$ & $\varepsilon$ & $\lambda_{\max }$ & $v_{\max }$ & $\varepsilon_{\max }$ \\
\hline $\begin{array}{l}1 \\
2 a \\
3\end{array}$ & $\begin{array}{l}3057 \\
2903 \\
2477\end{array}$ & $\begin{array}{l}98110^{12} \\
1033 * \\
1211\end{array}$ & $\begin{array}{c}18,02 \\
1422 \\
36160\end{array}$ & $\begin{array}{l}2891 \\
2567\end{array}$ & $\begin{array}{l}10 \overline{9} .10^{12} \\
1169\end{array}$ & $\begin{array}{r}\overline{1} \\
1322 \\
23320\end{array}$ \\
\hline
\end{tabular}

$1^{\circ}$ Forme " dite " cétonique :

$2^{\circ}$ Forme dite énolique dans l'éther :

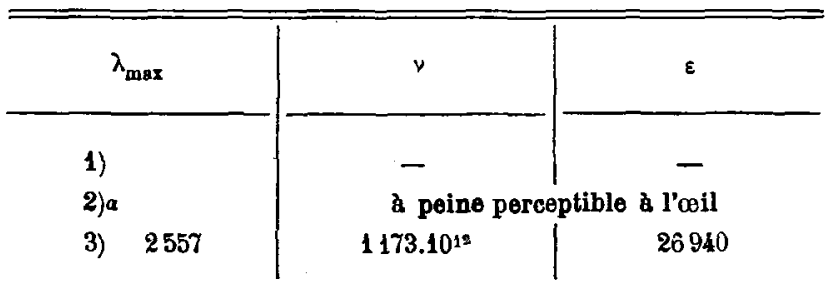

En solution hexanique la forme dite cétonique en plus d'une bande aromatique fine (bande 2 de notre tableau) possède deux bandes :

La première pour la fréquence $\nu=981.10^{12}$ se retrouve dans les dérivés pour lesquels la forme énolique est impossible. En comparant son intensité $\varepsilon=18,02$ à celle de la bande correspondante du diméthyle-diphényle-dicétocyclobutane (voir page 33) nous pouvons déduire qu'il y a 12 à $13 \%$ de forme dicétonique dans la solution.

$$
\frac{\varepsilon \text { diphényle- }}{\varepsilon \text { diphénylediméthyle- }}=\frac{18,02}{144,-}=0,125 \text {. }
$$

(1) K. H. MEYER, B., 47, p. 826 (1914). 
$2^{\circ}$ La deuxième bande très intense vers $\nu=1211.10^{12}$ avec $\varepsilon=36160$ est par son intensité et sa position très semblable à la forte bande de l'acétylacétone énolisé. Elle n'existe pas dans le spectre du diphényle-diméthyle-dicétocyclobutane qui ne peut être que dicétonique.

Cette bande se déplace vers les plus grandes longueurs d'ondes en solution alcoolique où $\nu_{\max }=1169.10^{12}$ environ. Son intensité est dans ce cas moins forte $\varepsilon=23320$.

Nous pouvons en conclure que la solution hexanique du diphényle-dicétocyclobutane forme dite cétonique est en réalité un équilibre de deux formes tautomères : dicétonique et énolique.

Ceci diffère de ce que Staudinger et Bereza $\left({ }^{1}\right)$, ont publié sur la constitution de cette combinaison. Ces auteurs n'ont pas admis l'existence de cet équilibre, basés. sur le fait que, par aucune réaction chimique, ils n'avaient pu déceler la présence de la forme énolique dans une solution de la dicétone $\left({ }^{2}\right)$.

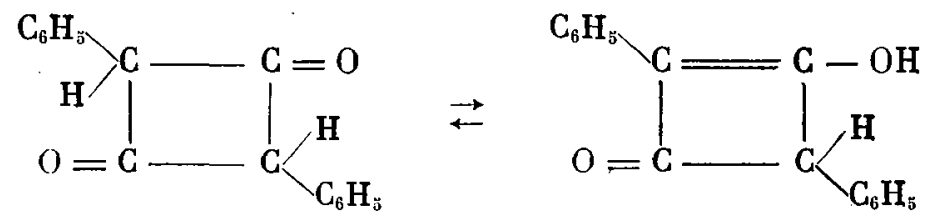

Par ses propriétés physiques, point de fusion et solubilités, la forme dite éno. lique se distingue complètement du produit dit cétonique. C'est un corps très instable à fort caractère acide monobasique.

On le prépare en traitant la forme dite cétonique avec de la soude caustique diluée. Un retour de la forme dite énolique à la forme dite cétonique, par un moyen quelconque, n'a jamais été observé. Le spectre d'absorption contient une bande très forte pour $\nu=1173.10^{12}$ et $\varepsilon=26940$.

Cette forme dite énolique à caractère acide est différente de la forme énolique que nous a révélé la spectrographie dans une solution hexanique de la forme dite cétonique.

On pourrait donner à la forme acide la formule d'un dicétocyclobutane diénolique :<smiles>OC1=C2C1(O)C2(c1ccccc1)c1ccccc1</smiles>

Ses solutions seraient formées d'un équilibre entre la forme monoénolique et diénolique :

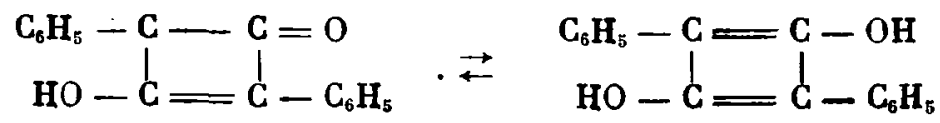

(1) B., 42, p. 4908 (1909).

(7) Une de ces solutions ne donne pas de coloration avec du FeClo. 
En admettant la formule spéciale de Hantsch pour les acides, nous pouvons aussi formuler cet équilibre comme suit ( $\left.{ }^{(}\right)$.

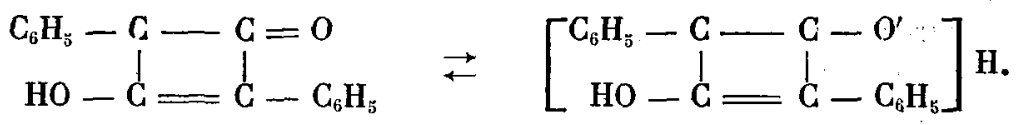

Nous ne donnons ces formules que comme hypothèses. afin d'exprimer l'observation nouvelle que nous avons pu faire sur la nature du diphényledicétocyclobutane.

3. $\beta$-dicétones n'existant que sous la forme énolisée. - Le diméthyledicétocyclobutane forme énolique (préparée par Schroeter).

$1^{\circ}$ Possède deux bandes en solution éthérée :

$$
\begin{array}{lll}
\lambda_{\max }=2706 \AA & { }_{\text {max }}=1108.10^{12} & \varepsilon_{\text {max }}=180,8 \\
\lambda_{\max }=2315 \AA & { }_{\text {max }}=1296.10^{12} & \varepsilon_{\text {max }}=17520
\end{array}
$$

$2 \circ$ Et en solution aqueuse une bande :

$$
\lambda_{\max }=2595 \AA \quad \nu_{\max }=1156.10^{\prime 2} \quad \varepsilon_{\max }=\overline{17520 .}
$$

La première correspond à celle de l'acétone avec $\nu=1108$. $10^{12}$.

La deuxième bande très forte est semblable à celle de l'acétylacétone bien que déplacée vers l'ultra-violet de $\Delta \nu=181.10^{22}$.

Ce déplacement de la bande énolique vers l'extrême $\boldsymbol{U}$. V. permet à la bande

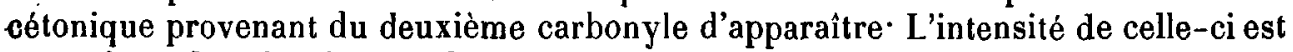
cependant plus de dix fois plus grande que celle de l'acétone. Cela peut provenir d'une diénolisation partielle.

La deuxième bande se déplace selon les solvants comme cela a déjà été observé pour l'acétylacétone.

$$
\begin{aligned}
& \Delta v=\text { Acétylacétone }: v_{\max } \text { hexane }-v_{\max } \text { eau alcaline }=115.10^{12} \\
& \Delta v=\text { diméthyl dic }: v_{\max } \text { éther }-v_{\max } \text { eau }=140.10^{12}
\end{aligned}
$$

Le rapport des intensités est :

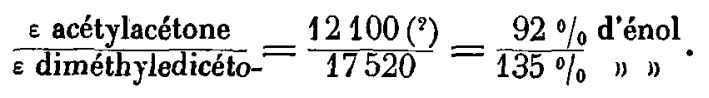

Il nous montre que le diméthylecyclobutane est totalement monoénolisé et probablement diénolisé en solution éthérée.

Cette diénolisation partielle n'a pas été démontrée par voie chimique. Le dimé-

(1) A. Hantsch, B., 50 (1917), p. 1422.

(2) Ces chiffres nous ont été communiqués par M. Victor Henri. 
thyledicétocyclobutane peut être titré comme un acide monobasique. Il est fort possible que l'iutensité particulièrement forte de la bande énol du diméthyledicétocyclobutane comparée à celle de l'acétylacétone ne provienne pas seulement de la diénolisation mais peut-être du cycle.

L'égalité de l'intensité d'absorplion en solution acqueuse et en solution éthérée montre que le diméthyle dicétocyclobutane n'existe pas sous la forme dicétonique.

4. Nous n'avons pas voulu classer le dimère du cétène dans l'un ou l'autre de ces groupes, car sa constitution n'a pas encore pu être déterminée exactement par l'analyse chimique. Par l'analyse de son spectre d'absorption nous pouvons. le classer avec le diphényle-dicétocyclobutane dans le groupe des $\beta$-dicétones énolisables.

Son spectre d'absorption se décompose en deux bandes :

\section{Solution hexanique}

\begin{tabular}{|c|c|c|c|c|c|}
\hline \multicolumn{3}{|c|}{ Iro bande } & \multicolumn{3}{|c|}{$2 \bullet$ bande } \\
\hline$\lambda$ on $\AA$ & $y$ & $\varepsilon$ & $\lambda$ en $\AA$ & $v$ & $\varepsilon$ \\
\hline $\begin{array}{l}\text { 1. } 3116 \\
\text { 2. } 2684\end{array}$ & $\begin{array}{r}969.1012 \\
1117 . \star *\end{array}$ & $\begin{array}{r}6,7 \\
27,7\end{array}$ & 233: & $1279.10^{12}$ & 90,31 \\
\hline
\end{tabular}

Solution ethérle

\begin{tabular}{|c|c|c|c|c|c|}
\hline \multicolumn{3}{|c|}{ ro bande } & \multicolumn{3}{|c|}{ 20 bande } \\
\hline$\lambda$ en $\AA$ & $v$ & $\varepsilon$ & $\lambda$ en $\AA$ & $v$ & $\varepsilon$ \\
\hline $\begin{array}{l}3116 \\
2684\end{array}$ & $\begin{array}{r}963.10^{12} \\
1117 . \times\end{array}$ & $\begin{array}{r}9,7 \\
27,7\end{array}$ & 2351 & $1276.10^{12}$ & 135,5 \\
\hline
\end{tabular}

La première bande est complexe, nous y observons deux maxima :

1. Le premier vers $\nu=963,10^{12}$ avec $\varepsilon=6,7$ en solution hexanique et 9,7 en solution éthérée.

20 Le second maximum pour $\nu=1117.10^{12}$ et $\varepsilon=27,1$ dans les deux solvants.

Cette bande est semblable à la première bande des dicétocyclobutanes totalement substitués ; elle est déplacée vers l'ultra-violet de $\Delta \nu=124.10^{12}$ en moyenne,' ce qui tient à l'absence de substituants quelconques aux groupes méthylènes.

Nous avons déjà observé ce déplacement vers le rouge, dû à l'introduction de 
radicaux aliphatiques ou aromatiques dans les cétènes. Victor Henri l'avait déjà mentionné dans son étude sur les cétones $\left({ }^{2}\right)$.

Comparée à l'intensité des dicétocyclobutanes substituées celle du dicétocy. clobutane paraît anormalement forte. Ceci peut provenir de la grande labilité du dicétocyclobutane.

Le diéthylecétène et le dipropylecétène nous donnent un exemple d'une absorption particulièrement forte d'un homologue inférieur moins stable que son homologue supérieur.

Enfin la deuxième bande dont l'intensité et la position du maximum varie selon le solvant peut être considérée comme la bande d'énol, très peu intense vu la petite concentration de cette forme.

En rapportant son intensité à celle de la bande correspondante du diméthyledicétocyclobutane nous trouvons pour la concentration en énol:

$$
\frac{\text { s dicétocyclobutane } \times 133 \%}{\varepsilon \text { diméthyledicétocyclobut. }}=\frac{90 \times 133}{17520}=0,0068 ; \quad \frac{135 \times 133}{17520}=0,0102 .
$$

Elle est de $0,68 \%$ en solution hexanique et de $1,02 \%$ en solution éthérée. La très petite quantité de forme énolique du dicétocyclobutane dans les solvants organiques est la raison pour laquelle elle n'a jamais pu être identifiée chimiquement.

Ces relations excessivement étroites entre le spectre d'absorption de l'acétone, du diméthylecyclobutane, forme énolique, du dimètre du cétène nous permet d'exclure comme symbole du $\left(\mathrm{H}_{2} \mathrm{C}=\mathrm{CO}\right)_{2}$ toute autre formule que celle indiquée par Staudinger dans son ouvrage sur les cétènes $\left(^{2}\right)$.

Rheiner a examiné toutes les possibilités de structure que présente le dimère du cétène $\left({ }^{3}\right)$, des dix formes possibles il a pu en éliminer six, les quatre autres sont :

Trois formes desmotropes et une forme lactone.

$$
\begin{aligned}
& \mathrm{H}_{2}=\mathrm{C}-\mathrm{C}=\mathrm{O} \\
& \mathrm{O}=\stackrel{\mathrm{C}}{\mathrm{C}}-\stackrel{\mathrm{l}}{\mathrm{C}}=\mathrm{H}_{2} \\
& \mathrm{O}=\mathrm{C}=\underset{\mathrm{C}}{\mathrm{C}}=\frac{\mathrm{COH}}{2} \mathrm{CH}^{2} \\
& \mathrm{HO}-\stackrel{\mathrm{C}}{\mathrm{HC}}=\mathrm{C}-\mathrm{C}-\mathrm{OH} \\
& \begin{array}{r}
\mathrm{H}_{2} \mathrm{C}=\mathrm{C}-\mathrm{C} \\
\mathrm{H}_{2} \mathrm{C}-\mathrm{l} \\
\mathbf{4}
\end{array}
\end{aligned}
$$

La dernière doit etre exclue, car le spectre n'a aucune analogie avec celui d'un éther d'un acide non-saturé pour lequel $\varepsilon$ est dans l'ultra-violet extrême supérieur à 10000 tandis qu'on a $\varepsilon$ égal à 135. Quant aux trois premières ce sont les formes tautomères d'un même corps. Nous pouvons conclure qu'une solution de

(1) Etudes de Photochimie et B., 46, p. 1930 (1913).

(2) Staudinger, Les Cétènes, p. 40.

(3) A. Rheiner, Thèse (Zurich, 1923, au laboratoire de M. Staudinger). 
dicétocyclobutane contient un équilibre entre la forme dicétonique et les formes énoliques, celles-ci en très faible proportion.

Nous ne voulons pas analyser ici le spectre d'absorption du<smiles>CCOC(=O)C1(C)C(=O)C2(C)C(=O)C1C2(C)OCC</smiles>

Nous nous bornerons à citer l'analogie de son spectre avec celui de l'oxalacétate d'éthyle en solution alcaline (voir partie expérimentale page 7). 
Leer - Vide - Empty 


\section{PARTIE EXPÉRIMENTALE}

\section{1. - METHODES G EN ERALES DE TRAVAIL}

Les spectres d'absorption des cétènes, de leurs dérivés et des autres combinaisons, qui par leur nature présentaient quelque intérêt pour nous, ont été étudiés en solution. Dans deux cas seulement nous avons photographié et mesuré les spectres à l'état de vapeur; cela a été pour le cétène $\mathrm{CH}_{2}=\mathrm{C}=\mathrm{O}$ et le diacétyle.

Les méthodes appliquées ont été celles que Victor Henri expose dans son Etudes de Photochimie ( $\left.{ }^{\mathbf{1}}\right)$ et plus récemment dans son article sur les spectres d'absorption et de fluorescence du Benzène $\left({ }^{2}\right)$. Sources lumineuses, spectrographes et produits photographiques employés par nous sont ceux qui y sont cités. Nous ne saurions les décrire à nouveau. Nous nous bornerons à indiquer dans ce paragraphe notre façon de procéder pour l'analyse spectrale d'un produit.

1 - Détermination qualitative de l'absorption par une plaque d'ensemble :

Pour cela nous avons interposé entre une source lumineuse et la fente d'un spectrographe, sur le chemin des rayons lumineux, une cuvette contenant la solution du corps à étudier. Nous avons photographié les spectres pour différentes concentrations (en général mol./10000 à mol/10) sous des épaisseurs variables en commençant par les plus petites.

Après examen de la plaque nous avions connaissance des régions intéressantes, dont l'étude devait être poussée plus loin.

20 Détermination quantitative de l'absorption pour le calcul du coefficient d'absorption moléculaire $\varepsilon$ :

Des épaisseurs égales de solution et de solvant pur sont alternativement photographiées à la lumière d'une étincelle avec des temps d'exposition différents mais connus. Les plaques ainsi obtenues sont examinées à la loupe afin de déterminer les points d'égalité de noircissement. La valeur du coefficient d'absorption molécu-

(1) Victor Henri, Etudes de Photochimie, Paris, 1919, chez Gauthier-Villars.

(8) Journal de Physique et le Radium, juin 1922, série VI, t. III, p. 181-211.

Guillaume C. Lardy. 
laire pour ces points, la longueur d'onde est donnée par celle des raies spectrales correspondantes, est calculée par la relation suivante :

$$
\varepsilon=\frac{k}{c}
$$

où $c$ est la concentration où $\varepsilon$ est le coefficient d'absorption moléculaire et $\mathrm{K}$ défini par la formule :

$$
\mathbf{I}=\mathbf{I}_{0} \cdot 10^{-k d}
$$

et calculé selon la loi du noircissement de Schwarzschild :

comme suit :

$$
\frac{\mathrm{I}_{0}}{\mathrm{I}}=\left(\frac{t}{t_{0}}\right)^{n}
$$

$$
\mathrm{K}=\frac{1}{d} \cdot \log \frac{\mathrm{I}_{0}}{\mathrm{I}}=\frac{n}{d} \cdot \log \frac{t}{t_{0}}=\frac{0.9}{d} \log \frac{t}{t_{0}}
$$

où $t_{0}$ est le temps d'exposition pour le solvant pur, $t$ pour la solution, $d$ l'épaisseur en centimètres, $n$ d'après les expériences de Victor Henri était égal à 0,9 pour les plaques que nous avons employées $\left({ }^{\mathrm{T}}\right)$.

Les temps d'exposition ont varié de 20 à 120 secondes selon la disposition des appareils et la région à étudier. Pour l'extrême ultra-violet nous utilisions la lumière d'une étincelle condensée entre électrodes de cuivre, et des plaques sensibilisées $\left(^{2}\right)$. Pour l'ultra-violet moyen et le visible nous avons travaillé avec des électrodes de $\mathrm{Fe}$ et de $\mathrm{Cd}$. tinu $\left({ }^{3}\right)$ :

$3^{\circ}$ Détermination exacte de la forme des bandes au moyen du spectre con-

La densité des spectrogrammes abtenus a été mesurée avec le microphoto. mètre de Fabry et Buisson ( ${ }^{*}$. Pour des bandes étroites nous avons photométré des points distants de $0^{\mathrm{mm}}, 2$; si les bandes étaient larges ils l'étaient de $0^{\mathrm{mm}}, 5\left(^{(}\right)$.

Toutefois i' n'y a concordance entre les valeurs du coefficient d'absorption moléculaire, trouvées par cette méthode et celles déterminées par les recherches

(1) Etudes de Photochimie, p. 7.

(2) Par le procédé de Duclaux et Jeantet, par immersion de la plaque dans une solution d'huile minérale dans de la benzine (voir V. Henri, Spectre d'absorption du benzène (loc, cit.).

(3) Procédé de Victor Henri pour l'obtention d'un spectre continu dans l'ultra-violet (Physik. Zeitsch., 14, 516, (1913) et loc. cit.).

(4) On entend par densité de la plaque le logarithme de l'apacité (Victor HenrI, Spectre d'absorption du benzène (loc. cit.), p. 182). vante :

(5) La dispersion du grand spectrographe de HIIGER que nous avons employé est la sui-

$$
\begin{aligned}
& \text { pour } \lambda=4000 \AA 1 \mathrm{~mm} \text { correspond } \mathrm{a} d \lambda=38 \AA \\
& \text { * } \lambda=3500 \AA \text { \# } \quad \text { a } d \lambda=24 \AA \\
& \text { - } \lambda=3000 \AA \quad \text { \# } \quad \text { a } d \lambda=16 \AA \\
& \text { » } \lambda=2500 \AA \text { A } \quad \text { à } d \lambda=9 \AA
\end{aligned}
$$


quantitatives que si le noircissement du spectre de comparaison a été mesuré sur le même spectre que celui où on détermine l'absorption. Pour cela on photomètre des points en debors des régions d'absorption ot on calcale les autres par interpolation. Ceci n'est possible que si les bandes ne sont pas réparties sur toute la longueur de la plaque. Sinon il faut recourir à un artifice, c'est-à-dire calculer un spectre de comparaison au moyen des valeurs de $\varepsilon$ trouvées avec le spectre d'étincelle.

Dans de nombreux cas nous avons été forcés de le faire. La concordance entre les valeurs interpolées et celles des mesures quantitatives était bonne.

Pour le tétra-éthyle-dicétocyclobutane, par exemple, nous avons trouvé :

$\begin{array}{ccc}\lambda \text { on } \AA & \begin{array}{c}\varepsilon \\ \text { par étincelle }\end{array} & \text { au Photomètre } \\ 3 \overrightarrow{600} & 5,3 & 5 \\ 3247 & 6,8 & 6,7 \\ 3180 & 8,8 & 9 \\ 2716 & 2,6 & 2,4\end{array}$

Comme le noircissement d'une plaque photographique n'est pas fonction linéaire du temps d'exposition, il n'était pas indifférent pour obtenir une forme aussi juste que possible de nos courbes de photométrer les divers points à des intensités variables. Nous avons fait en sorte de mesurer un même spectre dans des régions de noircissement sensiblement égal. Pour cela nous avons photométré les maxima sur les pholographies prises pour de petites épaisseurs et les minima d'absorption sur celles des épaisseurs plus grandes.

Etablissement des courbes des spectres d'absorption. - Nous avons construit les courbes d'absorption en portant sur des diagrammes les valeurs du logarithme du coefficient d'absorption moléculaire $\varepsilon$, défini par la relation $I=I_{0} 10-\varepsilon c d$, en ordonnée et en abcisse la fréquence $\nu$, égal au quotient de la vitesse de la lumière en $\mathrm{cm} / \mathrm{sec}$. par la longueur d'onde en $\mathrm{cm}$.

Elles sont directement comparables en conservant l'échelle aux courbes publiées par Victor Henri et ses collaborateurs.

Comparées à celles de Baly ou de Hantsch elles présentent des maxima où celles des auteurs ont des minima. Ceux-ci ont coutume de porter en ordonnée le logarithme des épaisseurs absorbantes et non pas comme nous la valeur de l'absorp. tion.

Mesure des spectres de vapeurs ( $\left.{ }^{(1}\right)$. - Nous avons photographié les spectres du cétène et du diacétyle à l'état de vapeur. 'On interpose pour cela entre la source lumineuse et la fente du spectrographe un tube de longueur connue contenant la vapeur sous une pression déterminée. Sans déplacer la plaque on photographie

(1) Victor Henri, Journal de Physique et le Radium, juin 1922, série VI, t. III, p. 181-211. 
successivement un spectre de la vapeur et un spectre repère d'étincelle fer-cadmium. La longueur d'onde des bandes observées est directement mesurable au microcomparateur ; elle est égale à celle de la raie du spectre repère qui lui est superposée.

Le tube que nous avons employé pour nos expériences avait 1 mètre de long. Les vapeurs y étaient introduiles par fractions successives exactement connues. En réunissant le tube avec un ballon de même volume nous pouviơns réduire la pression de $1 / 2,1 / 4,1 / 8$, etc.

Le vide est fait par une pompe de Langmuir en quartz montée en série sur une trompe à eau avec toutes les précautions nécessaires pour éliminer la vapeur d'eau. Les pressions pouvaient varier de 1/100 de millimètre à 65 millimètres de mercure.

\section{2. - PRÉPARATION DES SOLVANTS $\left({ }^{1}\right)$}

Une étude des spectres d'absorption jusque dans l'ultra-violet extrême (2000 Å) exige une pureté très grande des solvants.

Pour les cétènes les seuls solvants utilisables ont été l'éther et là benzine. Pour les dérivés tautomères comme certains dimères des cétènes nous avons employé en outre de l'alcool et de l'eau.

L'hexane (fraction de la benzine, distillant entre $62^{\circ}$ et $75^{\circ} \mathrm{C}$.) présente sur les autres solvants organiques de grands avantages. Il est relativement facile à nettoyer et absorbe même en couche épaisse très peu. Il a peu d'action sur la position des bandes d'absorption. La substance qui y est dissoute donne un spectre très sem. blable à celui qu'elle possède à l'état' de vapeur.

\section{A) Purification de l'hexane}

De la benzine rectifiée du commerce (P. E. $60^{\circ}$ à $75^{\circ}$ C.) est traitée pendant 24 heures avec de l'acide sulfurique à $96 \%$ puis avec de l'acide fumant (à $20 \%$ de $\mathrm{SO}_{3}$ ) pendant le mème laps de temps. On la lave ensuite avec de la soude caustique el l'agite avec une solution de permanganate de potasse acide. Après avoir été lavé à nouveau. séché et distillé sur du sodium métallique l'hexane est prêt à être essayé. Si ce traitement n'a pas suffit pour enlever les impuretés, on le chauffe pendant quelques heures à ébullition avec du chlorure d'aluminium anhydre. Sans vouloir entrer dans les détails, nous voulons mentionner qu'un traitement à l'acide nitrique ne donne pas de bons résultats.

(1) Les méthodes de préparation des solvants ont été étudiées et mises au point par M. F. W. Klingstedt, qui a bien voulu nous les communiquer et nous montrer la meilleure manière d'en faire usage. Nous tenons à l'en remercier ici ainsi que de tous les conseils qu'il nous a donné, tout spécialement, pour la purification de quelques-uns de nos produits. Son aide nous a souvent facilité le travail au cours de cette étude. 


\section{B) Purification de l'éther}

Il suffit de chauffer de l'éther absolu pendant quelques heures sur du sodium soigneusement nettoyé el de le rectifier.

\section{C) Purification de l'alcool méthylique}

L'alcool mélangé à de la soude caustique dans laquelle on a dissous de l'iode est abandonné un jour. On le verse alors lentement dans une solution de nitrate d'argent à $10 \%$ (le quart environ de la quantité d'alcool) et laisse l'oxyde d'argent se précipiter; puis on agite quelques heures. Apres distillation l'alcool rendu absolu $n \in$ présente plus d'absorption gênante. lique :

Cette méthode a été depuis modifiée pour la purification de l'alcool éthy-

$1^{\circ}$ Traitement à l'iode et distillation ;

- $2 \circ$ Distillation sur de la poudre de zinc.

\section{3. - SPECTRES D'ABSORPTION DES CETENES}

\section{a) Le cétène.}

Nous avons préparé le cétène par décomposition pyrogénée de l'acétone dans un courant d'azote $\left(^{\mathbf{1}}\right)$.

Les vapeurs d'acétone passent dans un tube en quartz rempli de tessons de porcelaine, chauffé dans un four électrique. Elles se condensent dans une série de récipients plongés dans des mélanges réf́rigérants. Les liaisons entre les différentes parties de l'appareil étaient faites avec des rodages soigneusement graissés. Aux deux extrémités du tube de quartz, nous avons été obligés d'employer des bouchons de caoutchouc. Pour éviter qu'ils ne soient en contact avec les vapeurs, nous les avions recouvert d'un enduit de $\mathrm{BaSO}_{3}$ séché et de papier d'étain.

Pour la décomposition la température a été maintenue entre $650^{\circ}$ et $660^{\circ} \mathrm{C} .\left(^{2}\right)$. Après avoir passé dans deux récipients refroidis à $-20^{\circ} \mathrm{C}$. et un à $-40^{\circ} \mathrm{C}$., où il abandonne sa teneur en acétone, le cétène se condense dans trois tubes en " $U$ " plongeant dans un mélange réfrigérant, neige de $\mathrm{CO}_{2}-$ éther $\left(-80^{\circ} \mathrm{C}\right.$.) .

La liqueur est presque incolore. En reliant le tube qui la contient à une nou-

(1) H. Staudinger, Les Cétènes, Stuttgart, chez Encke, 1912; Wilsmore et Stewart, Jour. Chem. Soc. (1907). Schmidin et Bergmann, B., 43, 2821 (1910).

$\left({ }^{2}\right)$ La température de décomposition la plus favorable a été déterminée par M. STiRneMANN : elle est égale à $650^{\circ}-655^{\circ} \mathrm{C}$. Le rendement, rapporté à l'acétone employé est de $11,3 \%$. Le cétène condensé est d'autant plus pur que la température est maintenue plus basse. Le produit est très fortement coloré en jaune si on travaille au-dessus de $800^{\circ} \mathrm{C}$. 
velle série de vases de condensation, nous sommes parvenus, après trois distillations successives, à obtenir un cétène tout à fait incolore.

La solution éthérée destinée à la spectrographie a été préparée par absorption du cétène dans de l'éther absolu.

Titrage de la solution :

Trois prises d'essai additionnées d'éther humide, nous ont fourni des solutions d'acide acétique que nous avons titrées avec de la lessive de baryte.

10 centimètres cubes de la solution éthérée ont été neutralisés par :

$1^{\circ} 8^{\mathrm{cm}^{3}}, 4 ; 2^{\circ} 8^{\mathrm{cm} 3}, 9$ de baryte $\mathrm{n} / 5$ (facteur $=0,9399$ ).

Nous avons titré le troisième échantillon après quelques jours; il a été neutralisé par $7^{\mathrm{cm}^{\mathrm{a}}}, \mathbf{8}$ de la même lessive $\left(^{\mathbf{1}}\right)$.

Le titre de la solution est d'environ 0,1777 moléculegramme par litre.

En additionnant 20 centimètres cubes de cette solution de $17^{\mathrm{cms}}, 3$ d'hexane, nous avons préparé les concentrations mol/10, mol $/ 100$, et $\mathrm{mol} / 1000$ de cétène, dont nous avọns photographié les spectres.

Les principales valeurs du coefficient d'absorption moléculaire sont les suivantes:

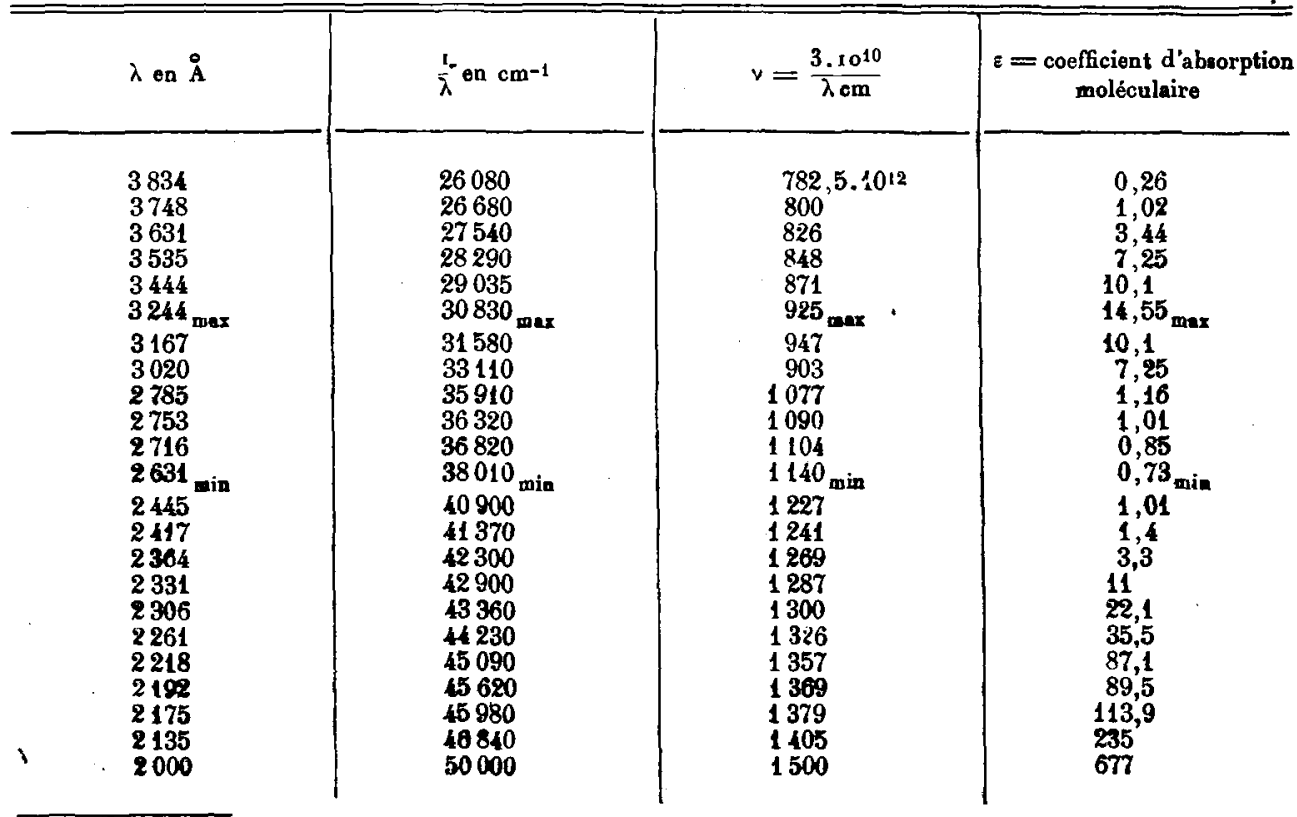

(4) M. Bodmer a essayé le solvant qui se prêtait le mieux à la détermination titrimétrique du cétène. Entre la benzine et l'éther, c'est l'éther qui est le plus favorable. Les résultats de l'analyse sont d'autant plus justes que la titration se fait aussitôt la solution préparée. Après quelques heures la concentration en cétène diminue.

Exemple : 1,530 gramme de cétène sont dissous dans 200 centimètres cubes de benzine. Théotiquement 25 centimètres cubes devraient employer 45,5 centimètres cubes de lessive de baryte. On a employé $44,2,43,4$ et 44,6 centimètres cubes.

Dans l'éther : théoriquement 23,8 centimètres cubes ; ont été employés: 23,$5 ; 23,3$ et 23,3 centimètres cubes de lessive de baryte $n / 10$. 


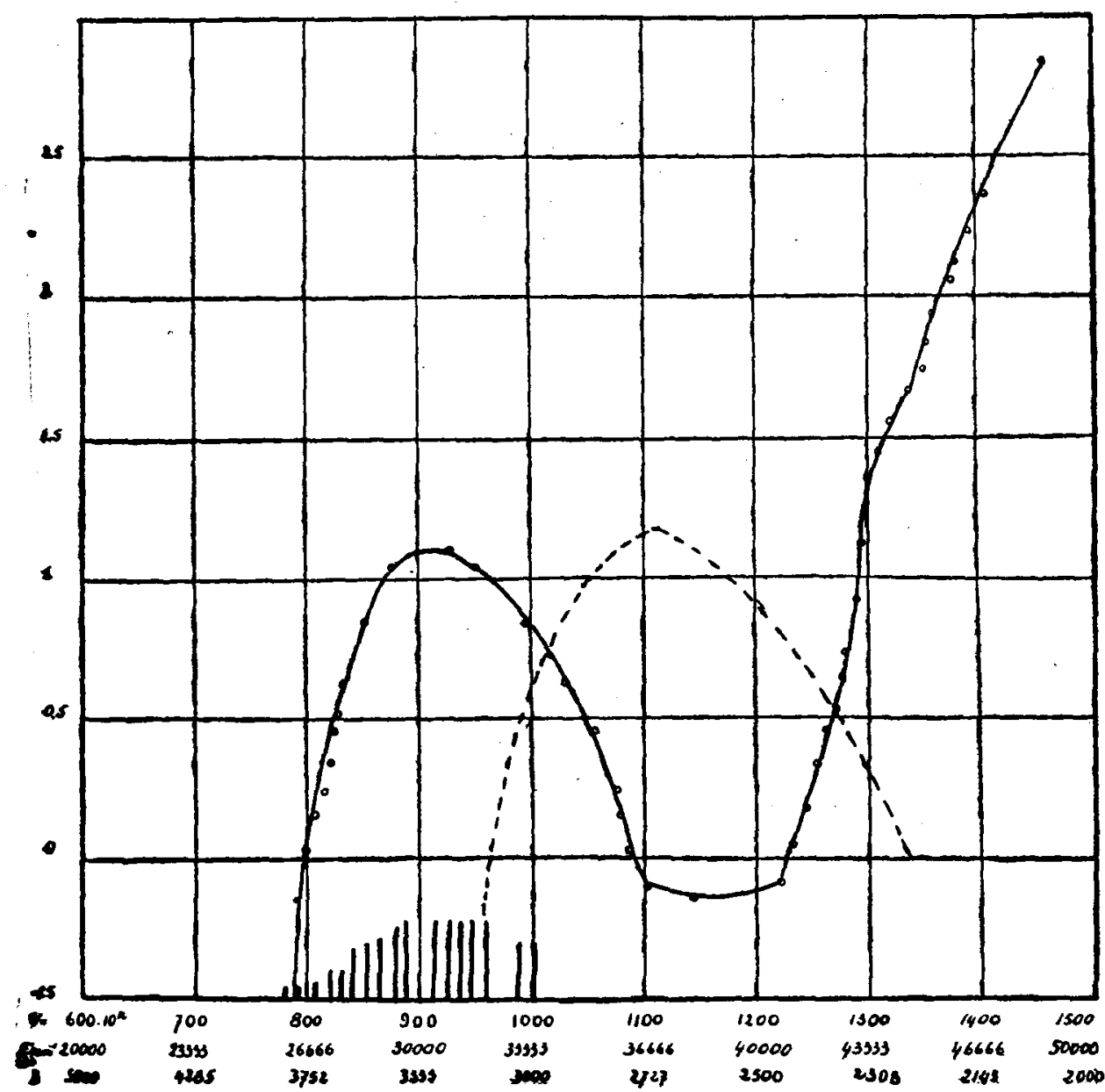

Fig. 1. - Spectre d'abeorption dn Cstène $\mathrm{CH}_{2}=\mathrm{C}=0$.

1. Trait plein : solution hexanique du cétèné

2. Trait pointillé : Acêtone en solution d'après Victor Henri.

Les bandes du spectre de vepeur du cótłne sont représentées par les petits traits verticaux an bas de la planche. Lcur hautour est proportionnelle à leur intensité relative. 
La courbe se compose de deux bandes :

La première a son maximum pour

$$
\lambda=3244 \AA \quad v=925.10^{12} \quad \varepsilon=14,5
$$

La largeur $\left.{ }^{(1}\right)$ est égale à $\Delta \nu=144.10^{18}$ et sa surface moyenne (largeur $\left.\times \frac{1}{2} \varepsilon_{\max }\right)$ à 2095. Elle est presque symétrique.

Comparée à celle de l'acétone elle lui est très semblable mais déplacée vers le spectre visible de $\Delta \nu=184.10^{12}\left({ }^{2}\right)$. Cette bande peut être attribuée au groupe $\mathrm{C}=\mathrm{O}$.

La seconde bande a son maximum dans l'ultra-violet extrême, pour une fréquence plus grande que $1500.10^{12} \varepsilon>677$. Nous n'avons pas pu en déterminer la position exacte. Cette bande doit être attribuée au groupe $>\mathrm{C}=\mathrm{C}<$.

\section{Spectre de papeur.}

Tandis que l'acétone et les autres combinaisons qui n'ont qu'un chromophơre, possèdent à l'état de vapeur des bandes larges qui ne se subdivisent pas en bandes fines, le cétène possède à l'état de vapeur une série de bandes fines qui occupent la même place que la bande large de sa solution appartenant au groupe $\mathrm{C}=0$. Ce sont :

Ces bandes sont difficiles à mesurer, car elles sont assez peu nettes. Leur bord se trouve du côté de l'ultra-violet. Elles sont dégradées vers le rouge (les longueurs d'onde portées dans les tables sont celles des bords ultra-violets).

La différence des fréquences entre les bandes n'est pas constante; elle semble posséder deux valeurs selon qu'on passe d'une bande impaire à une bande paire ou inversement.

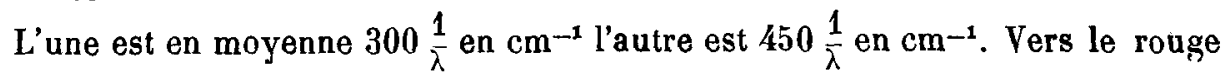
ces différences ne s'alternent plus avec la même régularité.

La différence des fréquences entre deux bandes impaires est égale à celle entre deux paires; elle est en moyenne de $\frac{1}{\lambda} \mathrm{cm}^{-1}=750$ et croît avec la longueur d'onde.

Au point de vue de l'origine de ces bandes fines on peut dire, conformémeñt aux théories modernes qu'elles sont dues aux vibrations des atomes dans le groupe absorbant $\cdot \mathrm{C}=0$. La tréquence de la vibration serait de l'ordre de $\frac{1}{\lambda}=450 \mathrm{~cm}^{-1}$ correspondant à $\lambda=22 \mu$.

(1) On entend par largeur d'une bande la différence de fréquences entre les deux points qui ont un coefficient d'absorption moléculaire égal à la moitié de celui du maximum.

(2) D'après VICton HeNri, l'acétone possède les valeurs suivantes : $\lambda_{\max }=2706 \mathrm{~A} v_{\max }=$ $1109.10^{12}, \varepsilon_{\max }=15,8$. La largeur est égale à $\Delta \varphi=169.10^{12}$ et la surface moyenne à 2671 . 


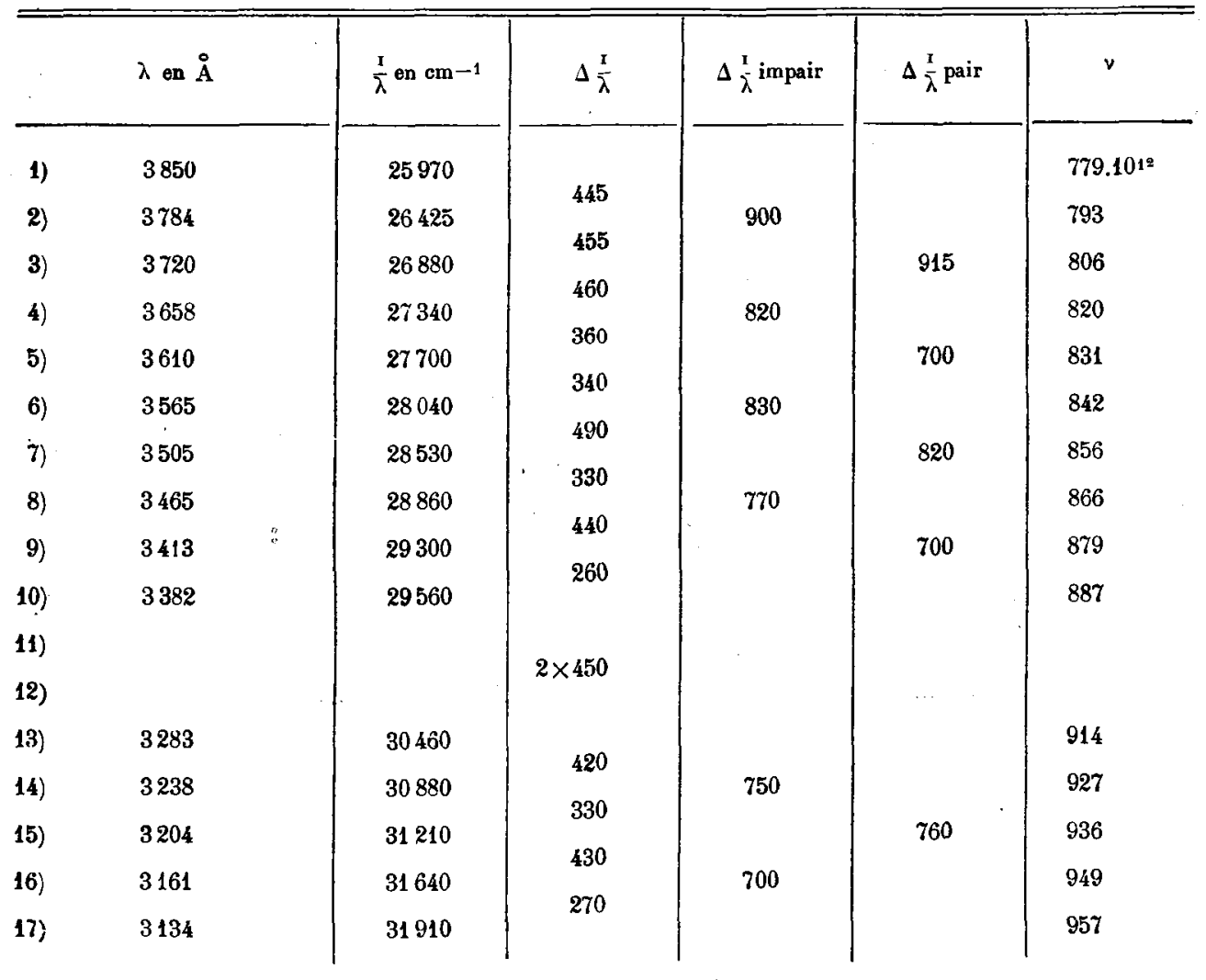

b) le diéthylecétène.

Le diéthylecétène ( $\left.{ }^{\boldsymbol{}}\right)$ a été préparé par décomposition pyrogénée de l'anhy dride mixte de l'acide malonique diéthylé et de l'acide diphénylacétique, $\left({ }^{2}\right)$. On peut aussi l'obtenir en traitant de l'acide diéthylemalonique avec de l'anhydride acétique. Ce procédé ne livre pas un produit très pur; nous lui avons préféré lautre (").

Nous avons fait réagir dans l'éther absolu des quantités équivalentes de diphénylecétène et d'acide diéthylemalonique.

L'anhydride mixte est solide ; on peut facilement le purifier par cristallisation dans de la benzine.

L'appareil avec lequel nous avons opéré la décomposition, permettait de distiller plusieurs fois le cétène sans devoir le transvaser à l'air libre. Les échantillons ont été pris et pesés dans de petites ampoules soudées à la lampe, semblables à celles qu'on emploie pour la combustion. Les solutions pour la specto-

(1) H. Staudinger, Les Cétènes, p. 142.

(2) H. Staudinger et Otт, Ber., 41, 2208 (1908).

(3) H. Staudinger et Ott, Ber., 41, 3829 (1908) et J. F. Félix, Thèse, Zurich, 1923. 
graphie furent préparées en brisant chaque ampoule dans la quantité d'hexane nécessaire pour une concentration mol/10. $\left(^{\mathbf{1}}\right)$.

$0,1896 \mathrm{grs}$ de diéthylecétène ont été dissous dans $19,35 \mathrm{~cm}^{3}$ d'hexane (sol. $\mathrm{mol} / \mathbf{1 0}$ ).

$0.103 \mathrm{grs}$ de diéthylecétène (P. E. $89,5^{\circ}-90^{\circ}$ à $724 \mathrm{~mm} \mathrm{Hg}$ de pression) ont été dissous dans $21 \mathrm{~cm}^{\mathrm{a}}$ d'hexane (sol. mol/20).

Ayant observé qu'en solution mol/10 un trouble se formait nous avons travaillé en solution mol/20. Le précipité fut moins abondant. Nous ne saurions dire en quelle proportion il s'est formé. Il faut en conséquence apporter une légère correction aux valeurs du coefficient d'absorption moléculaire que nous avons trouvées. Le précipité étant insoluble dans la benzine n'a pas absorbé de lumière. Seule la concentration en cétène a diminué. Le coefficient théorique doit être plus grand que le nôtre.

Nous avons photographié les spectres soit d'étincelle soit continus.

Voici les valeurs de $\varepsilon$ pour les différentes longueurs d'ondes:

\begin{tabular}{|c|c|c|c|}
\hline$\lambda$ en $\AA$ & $\frac{1}{\lambda}$ on $\mathrm{cm}^{-1}$ & $v=\frac{3.10^{10}}{\lambda \mathrm{cm}}$ & $\varepsilon$ \\
\hline $\begin{array}{l}4383 \\
4143 \\
4045 \\
3799_{\text {max }} \\
3670_{\text {max }} \\
3403 \\
3250 \text { miu } \\
3002 \\
2506 \\
2439 \\
2356 \\
2242_{\text {max }} \\
2025\end{array}$ & $\begin{array}{l}22810 \\
24135 \\
24780 \\
26320_{\max } \\
2 i 250_{\max } \\
29390 \\
30780_{\min } \\
30310 \\
39900 \\
41000 \\
42400 \\
44600_{\max } \\
49380\end{array}$ & $\begin{array}{l}654.1012 \\
724 \\
742 \\
790_{\max } \\
818_{\max } \\
881 \\
923_{\min } \\
999 \\
1197 \\
1230 \\
1273 \\
14338_{\max } \\
1481\end{array}$ & $\begin{array}{c}1,7 \\
7,23 \\
9,3 \\
17,5_{\max } \\
17,5_{\max } \\
9,3 \\
5,4_{\text {min }} \\
5,4 \\
13,9 \\
70 \\
350 \\
361_{\max } \\
2694\end{array}$ \\
\hline
\end{tabular}

La courbo se compose de deux bandes :

La première a pour $\lambda=3735 \AA$ et $\nu=803.10^{12}$ un maximum avec $\varepsilon=17,5$. Sa largeur est $\Delta \nu=150.10^{12}$ et sa surface moyenne 2625 .

Elle est symétrique. Blle est produite par le groupe $\mathrm{C}=0$.

La deuxième est vingt fois plus intense, elle se trouve vers

$$
\lambda=2242 \AA \quad \vee=1338.10^{12} \quad \text { et } \quad \varepsilon=361 .
$$

Sa largeur environ égale à $\Delta \nu=166.10^{12}$ : elle appartient au groupe $>\mathrm{C}=\mathrm{C}<$ La différence de tréquence entre les deux bandes est $\Delta \nu=535.10^{23}$.

(1) Toutes ces manipulations ont été faites après avoir séché les ballons et les flacons avee du $\mathrm{P}^{\curvearrowright}$ et les avoir remplis de gaz carbonique avec le plus grand soin.

(2) Ce précipité est vraisemblablement dû à l'auto-oxydation et provient de l'oxygène qui se trouvait dans l'hexane (?). Voir H. Stadoriger, Las Cétènes, p. 5 f. $_{\text {. }}$ 


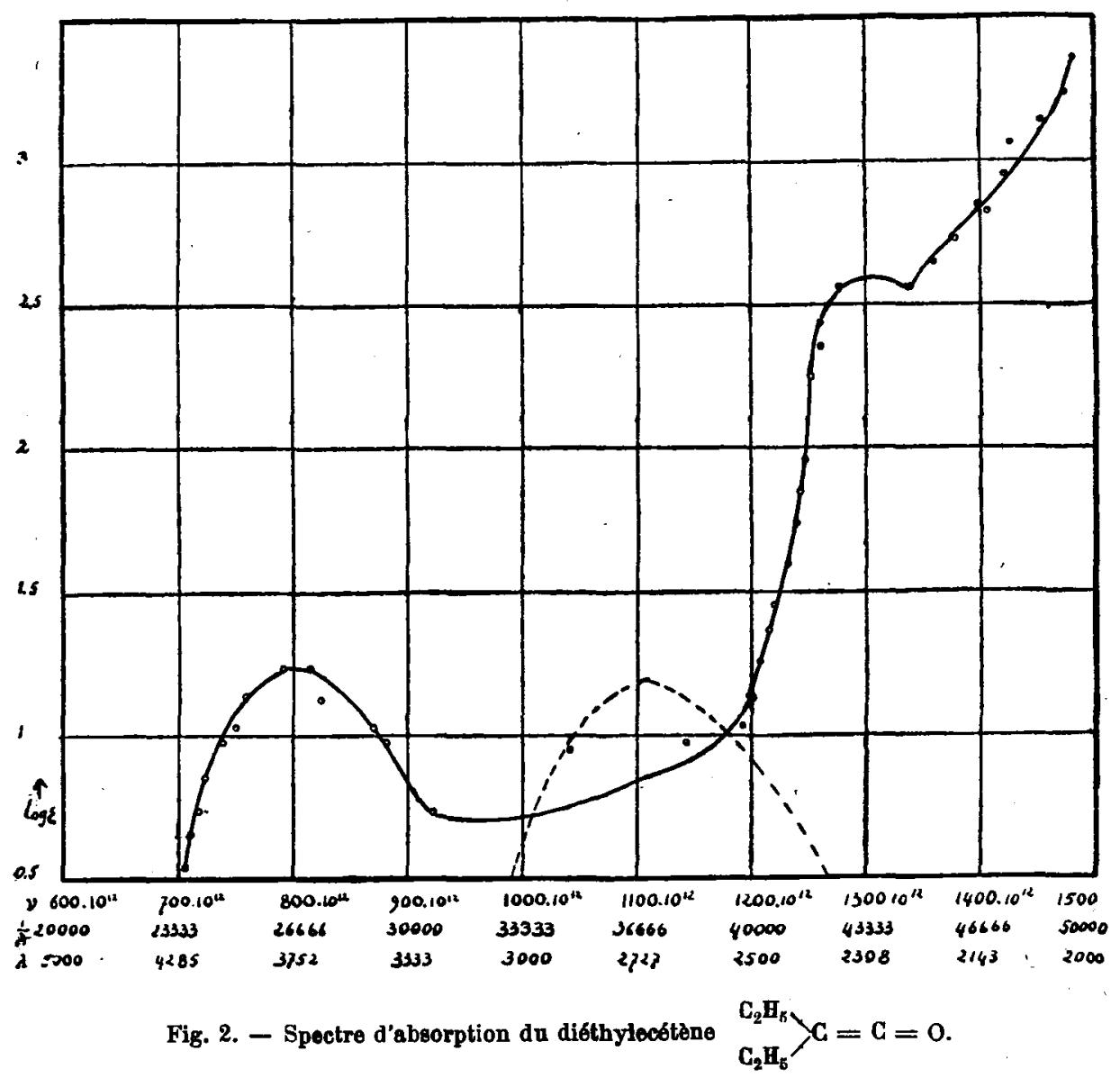

1. Trait plein : en solution hexanique.

2. Trait pointillé : Acétone d’après Victor Henri. 


\section{c) Le dipropylecétène.}

Nous avons procédé comme pour le diéthylecétène. L'anhydride mixte de l'acide dipropylemalonique et de l'acide diphényle-acétique, soigneusement purifié, a été chauffé jusqu'à sa décomposition totale en dipropylecétène ( ${ }^{1}$ ). Celui-ci distillé deux fois et fractionné a été pris en ampoules et pesé.

(1) $0,2350 \mathrm{gr} .\left(\right.$ P. E. $38^{\circ}$ C. à $14 \mathrm{~mm} \mathrm{Hg}$ de p.) ont été dissous dans $18,65 \mathrm{~cm}^{3}$ d'hexane.

(2) $0,2402 \mathrm{gr}$. ont été dissous dans $19,06 \mathrm{~cm}^{3}$ d'hexane.

(3) $0,4719 \mathrm{gr}$. ont été dissous dans $36,86 \mathrm{~cm}^{3}$ d'hexane.

Avec ces trois solutions nous avons photographié les spectres soit avec l'étincelle soit avec la lumière continue. suivantes :

Nous avons trouvé pour le coefficient d'absorption moléculaire les valeurs

\begin{tabular}{|c|c|c|c|}
\hline$\lambda=$ en $\AA$ & ${ }_{\lambda}^{1}$ en $\mathrm{cm}^{-1}$ & $\nu=\frac{\mathbf{c}}{\lambda}$ en cm & $\varepsilon$ \\
\hline $\begin{array}{l}4600 \\
4071 \\
3995 \\
3930 \\
3820 \\
3792_{\max } \\
3610 \\
3427 \\
3350 \\
3250 \\
3158_{\text {min }} \\
2996 \\
2483 \\
2356 \\
2315 \\
2190_{\max } \\
1944\end{array}$ & $\begin{array}{l}21740 \\
24560 \\
28030 \\
25445 \\
26180 \\
26380 \text { max } \\
27700 \\
29180 \\
29850 \\
30780 \\
31670_{\min } \\
33380 \\
40270 \\
42440 \\
43200 \\
45666_{\max } \\
51450\end{array}$ & $\begin{array}{l}65210^{12} \\
736 \\
751 \\
763 \\
785 \\
791_{\max } \\
831 \\
875 \\
895 \\
923 \\
950 \text { min } \\
1001 \\
1208 \\
1273 \\
1295 \\
1369_{\max } \\
1543\end{array}$ & $\begin{array}{c}0,89 \\
4,6 \\
6,77 \\
8,9 \\
11,7 \\
13,54_{\max } \\
11,7 \\
6,77 \\
5,4 \\
3,5 \\
2,3_{\min } \\
2,3 \\
11,7 \\
177 \\
271 \\
355_{\max } \\
3520\end{array}$ \\
\hline
\end{tabular}

La courbe présente deux maxima. La bande la moins intense a presque la même position que'celle du diéthylecétène; son maximum se trouve vers $\nu=791.10^{12}$ et $\lambda=3792 \AA ; \varepsilon=13,54$.

Sa largeur est égale à $\Delta \nu=124.10^{12}$ et sa surface moyenne à 1680 . Elle provient du groupe $\mathbf{C}=\mathbf{0}$.

Vers $2190 \AA$ nous observons le second maximum pour une fréquence $\nu=1369.10^{12}$ avec $\varepsilon=355$, une intensité plus de 95 fois plus forte que la première. Elle est due au groupe $>\mathrm{G}=\mathrm{G}<$.

La différence des fréquences entre les deux têtes de bandes est de $\Delta v=578.10^{12}$.

Bien que le dipropyle-cétène soit l'homologue supérieur du diéthyle, son

(1) H. Staudinger et P. Strong, Helv. Chim. Acta, VI, 296 (1923). 


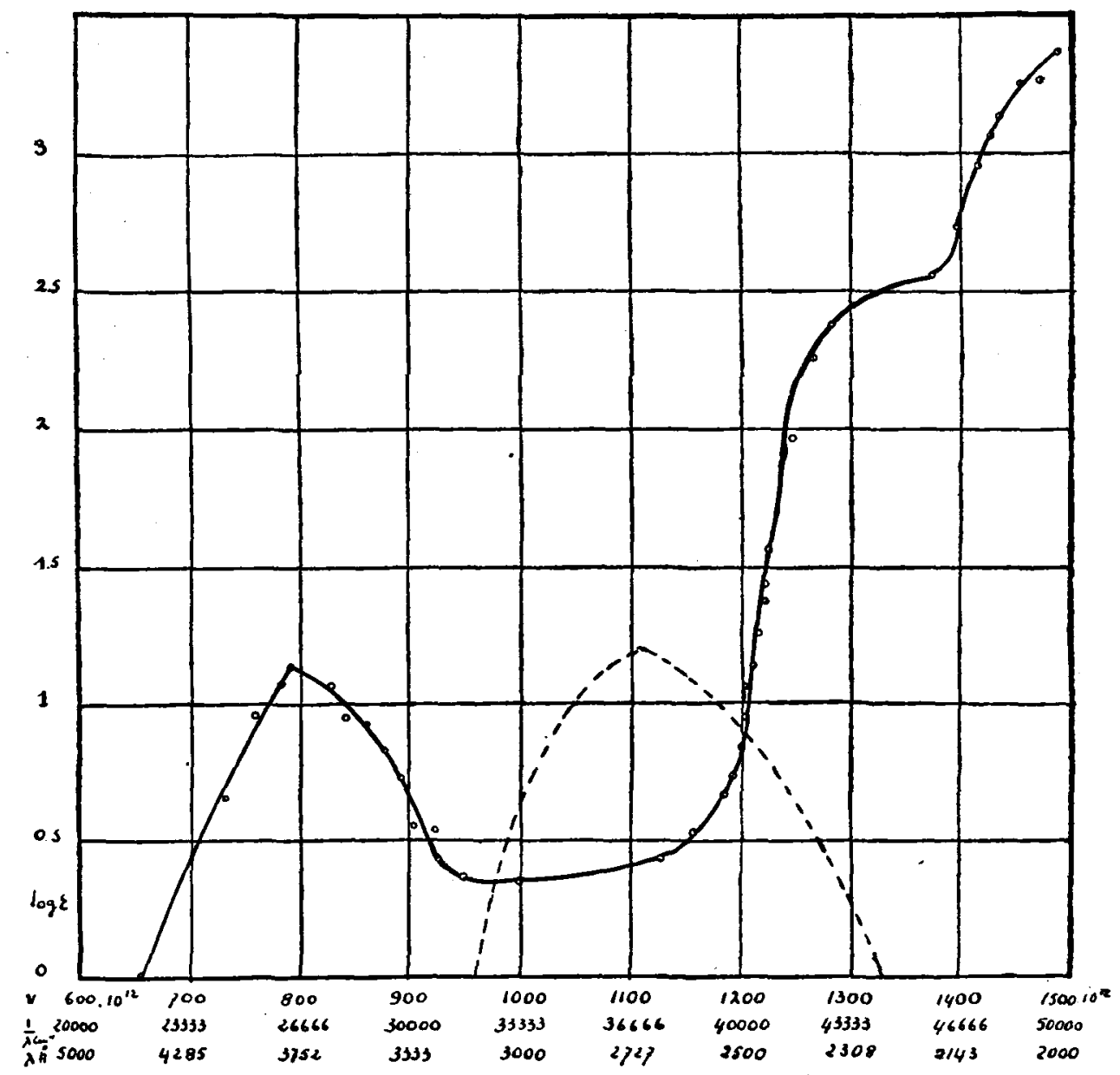

Fig. 3. - Spectre d'absorption du dipropylecétène $\left(\mathrm{C}_{3} \mathrm{H}_{7}\right)_{2}=\mathrm{C}=\mathrm{G}=0$.

1. Trait plein : en solution hexanique.

2. Trait pointillê : Acétone en solution d'après Victor Henri. 
intensité d'absorption est plus faible. Ceci est en accord avec la loi de la labilité chimique de Victor Henri, (loc. cit.) car dans toutes ses réactions le diéthylecétène est moins stable que le dipropyle et se transforme plus rapidement ( ${ }^{1}$ ).

\section{d) Le diphényle-cétène.}

Par la méthode de préparation de Schroeter ( $\left.{ }^{2}\right)$ le diphénylecétène est devenu un corps facile à obtenir. Pour le purifier nous l'avons fractionné à plusieurs reprises dans le vide d'une pompe à mercure.

Des échantillons ont été pris dans de petites ampoules, pesés et analysés. (P. E. $99^{\circ}$ à $0,01 \mathrm{~mm}$ de $\mathrm{Hg}$ de p.). Le degré de pureté du cétène était de 99,3 à $99,5 \%(3)$.

Solutions pour la spectrographie :

$0,2639 \mathrm{gr}$. de diphénylecétène ont été dissous dans $13,63 \mathrm{~cm}^{3}$ d'hexane (sol. $\mathrm{mol} / \mathrm{10}$ ).

$0,2429 \mathrm{gr}$. dans $12,51 \mathrm{~cm}^{3}$ d'hexane (sol. $\mathrm{mol} / 10$ ).

$0,3236 \mathrm{gr}$. dans $16,34 \mathrm{~cm}^{3}$ d'hexane (sol. mol/10).

La solution mol/10 ne se conserve pas. Après quelques minutes elle se trouble par la formation d'un oxyde moléculaire ( $)$. Mais l'absorption du diphénylecétène est assez intense pour qu'il suffise de photographier la solution molaire/100 pour étudier tout le spectre.

La courbe d'absorption du diphénylecétène se compose de deux maxima très distincts. Elle ne présente aucune trace de bandes aromatiques. Les valeurs mesurées sont les suivantes:

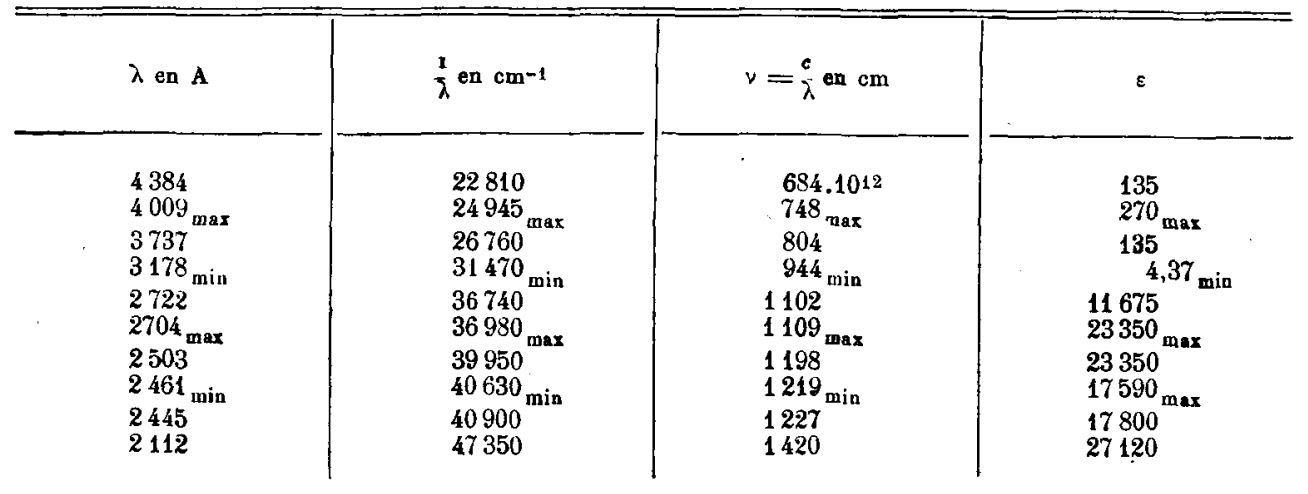

(1) H. Staudinger et P. Strong, Helv. Chim. Acla, VI, 296 (1923).

(2) Schroeter, B., 42, 2336 (1909) et Staudinger, B., 44, 1619 (1911).

(3) Ces analyses ont été faites par M. P. Richard, dans son travail sur la réaction des cétènes avec les alcools primaires. Il avait préparé le diphénylecétène selon nos indications.

(4) H. Staudinger, Les Cétènes, p. 49.

Nous avons pu identifier cet oxyde à sa solubilité et son virement au rouge dans les alcalis (réaction de l'acide benzilique). 


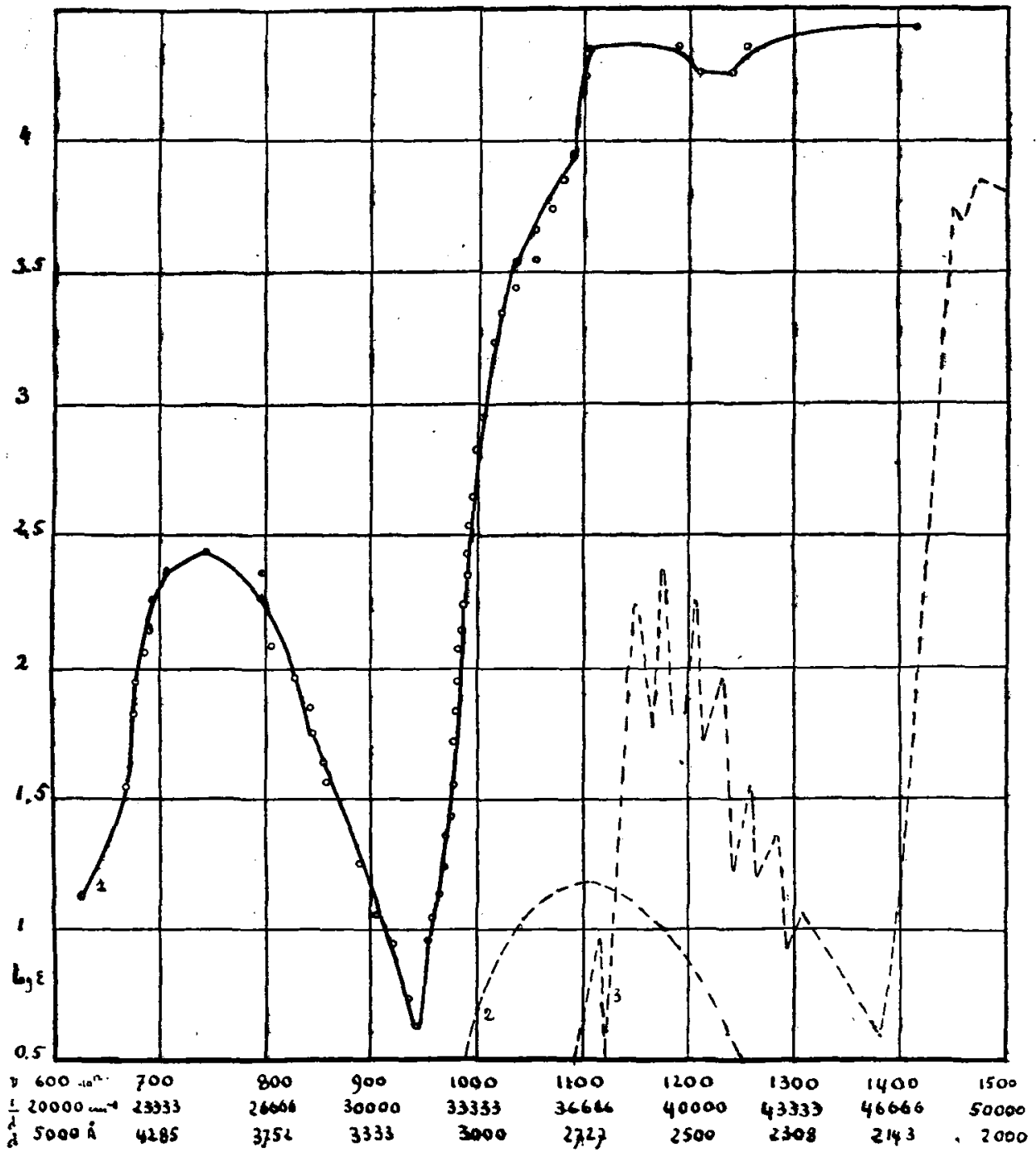

Fig. 4. - Spectre d'absorption du diphénylecétène $\left(\mathrm{C}_{6} \mathrm{H}_{5}\right)_{2} \mathrm{C}=\mathrm{CO}$.

1. Trait plein : en solution hexanique.

2. Trait pointillé : Acétone d'après Victor Henri.

3. Trait pointillé : Benżne en solution d'après Victor Henri. 
Le maximum de la bande du visible se trouve vers

$$
\lambda=4009 \AA \quad \nu=748.10^{12} \quad \text { avec une valeur de } \quad \varepsilon=270 .
$$

La largeur est égale à $\Delta \nu=119.10^{12}$ et sa surface moyenne à 32150 .

L'intensité très forte de la bande du groupe $\mathrm{C}=\mathrm{O}$ est dûe à la substitution aromatique. Elle correspond à des propriétés très spéciales du diphénylecétène, à la labilité caractéristique du chromophore $\mathrm{C}=\mathrm{O}$.

La deuxième bande a son maximum entre $\lambda=2704 \AA$ et $\lambda=2509 \AA$ pour une fréquence moyenne de $\nu=1154.10^{12} ; \varepsilon=23350$.

La différence des fréquences entre les têtes des deux bandes est égale à $\Delta \nu=406.10^{12}$.

Pour nous assurer que la première bande du diphénylecétène proviont du groupe $C=O$, nous avons mesuré le spectre du diphényléthylène asymétrique.

$$
\begin{aligned}
& \left(\mathrm{C}_{6} \mathrm{H}_{5}\right) \\
& \left(\mathrm{C}_{6} \mathrm{H}_{5}\right)
\end{aligned}>\mathrm{C}=\mathrm{CH}_{2}\left({ }^{1}\right)
$$

$1,1215 \mathrm{gr}$. (P. E. $145^{\circ}-146^{\circ}$ à $8 \mathrm{~mm} \mathrm{Hg}$ de p.) sont dissous dans $6,8 \mathrm{~cm}^{3}$ d'hexane (sol. mol./1)

$0.3892 \mathrm{gr}$. sont dissous dans $21,6 \mathrm{~cm}^{3}$ d'hexane (sol. mol/10).

Les principales valeurs mesurées sont les suivantes :

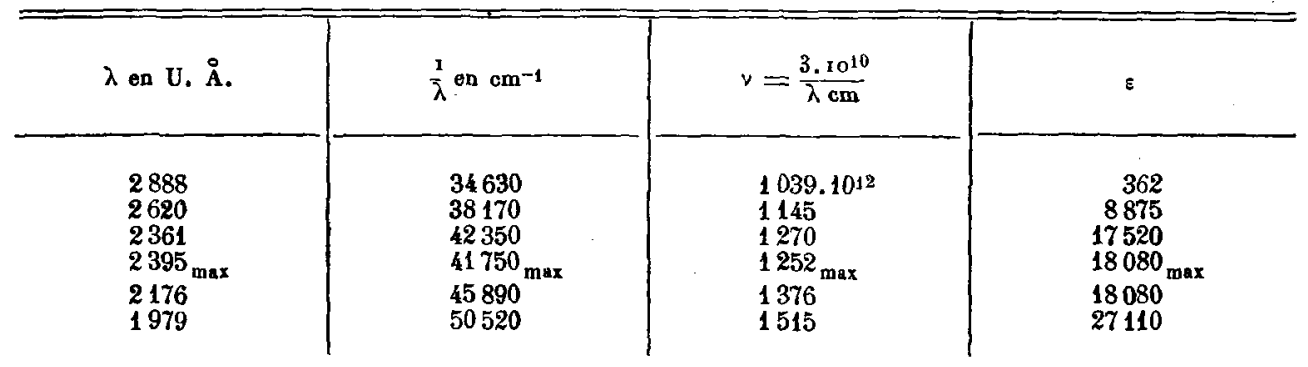

La courbe d'absorption dụ diphẻnyléthylène asymétrique est formé d'une large bande dont le maximum se trouve dans l'ultra-violet extrême.

Entre $\lambda=2395 \AA$ et $\lambda=2179 \AA$, pour $\nu=1315.10^{12}$ et $\varepsilon=18080$.

Bien qu'il possède deux noyaux aromatiques sa courbe d'absorption ne présente pas de bandes aromatiques.

(1) M. J. Tschuı nous a préparé le diphényléthylène asymétrique, comme l'indique TreFenau (Thèse de Suter, p. 107, Zurich, 1920).

Il a fait réagir de l'acétophénone sur du bromure de phényle-magnésium poú obtenir après saponification le diphényle-méthyle-carbinol. Celui-ci se transforme en dérivé éthylénique par l'action du gaz chlorhydrique sur sa solution dans l'acide acétique glacial.

Le rendement total est d'environ $55 \%$. 


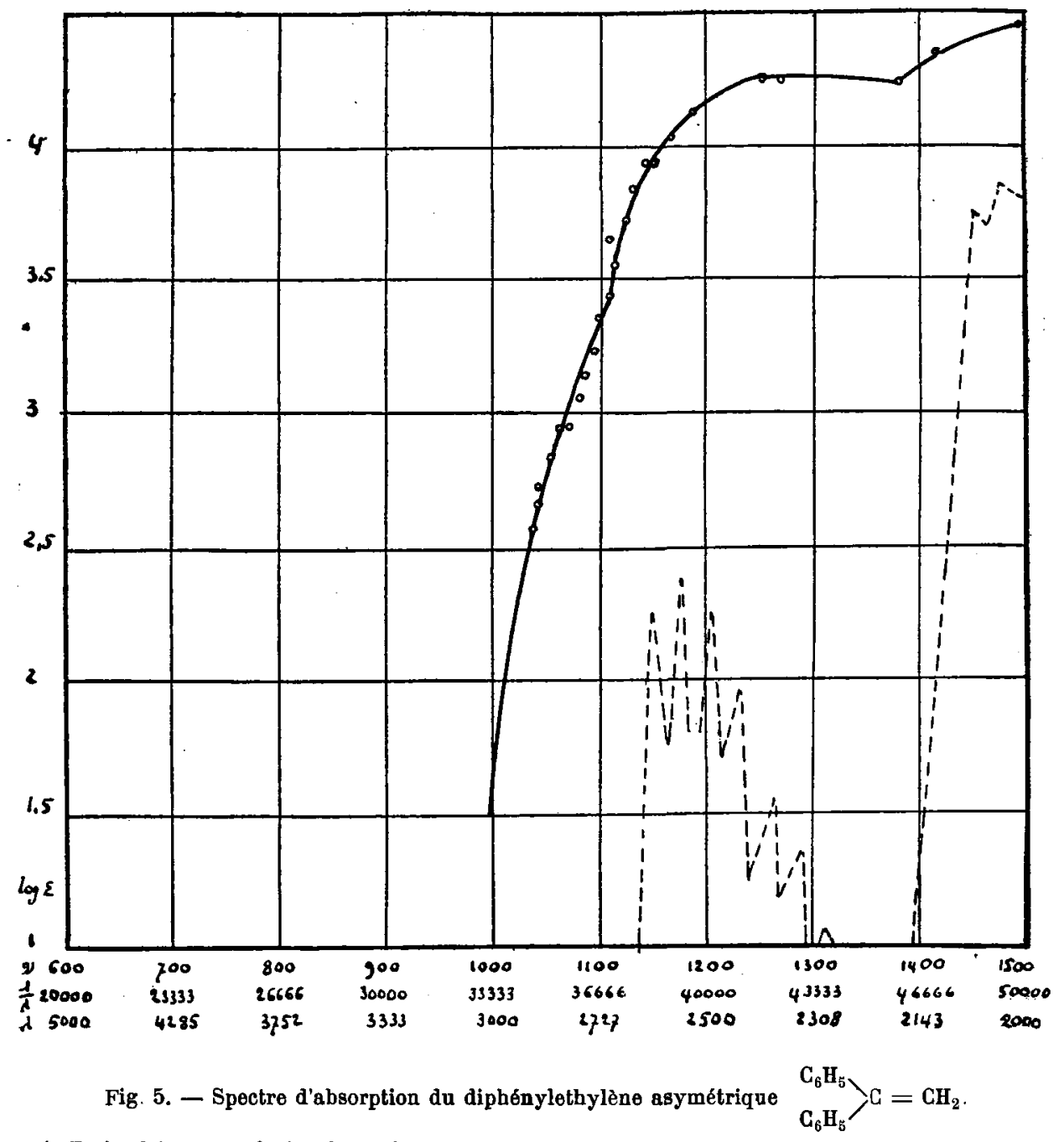

1. Trait plein : en solution hexanique.

2. Trait pointillé : Benzène en solution d'après Victor Henri.

Guillaume C Lardy. 
La bande du visible du diphénylecétène est absente. Notre hypothèse que cette bande est due au groupe $\mathrm{C}=0$ est par conséquent justifiée.

La large bande du diphényléthylène asymétrique provient de la liaison éthylénique et des groupes aromatiques. Elle ressemble aux bandes trouvées par Armand Castille pour une série de dérivés à 2 noyaux benzèniques.

\section{4. - SPECTRES D'ABSORPTION DES DIMERES DES CETENES (NON-DESMOTROPES)}

\section{a) Le dicétocyclobutane tétra-éthylé.}

S'obtient par polymérisation du diéthylecétène à la chaleur $\left(^{1}\right)$. Nous l'avons purifié par tractionnement dans le vide. (P. E. $108^{\circ}-109^{\circ}$ pour $20 \mathrm{~mm} \mathrm{Hg}$ de p).

$0,3176 \mathrm{gr}$. (P. F. 24. G.) ont été dissous dans $17,75 \mathrm{~cm}^{3}$ d'hexane (sol. mol/10).

Les valeurs de $\varepsilon$ pour les différentes longueurs d'ondes sont les suivantes:

\begin{tabular}{|c|c|c|c|}
\hline$\lambda$ en $\AA$ & $\frac{1}{\lambda}$ en $\mathrm{cm}^{-1}$ & $v=\frac{3.15^{10}}{\lambda \mathrm{cm}}$ & $\varepsilon$ \\
\hline $\begin{array}{l}3636 \\
3444_{\max } \\
3234 \\
3178 \\
3142_{\max } \\
3113_{\max } \\
2806 \\
2891_{\max } \\
2643_{\min } \\
2424 \\
2356 \\
2036_{\max } \\
1989\end{array}$ & $\begin{array}{l}27500 \\
29035_{\max } \\
30920 \\
31470 \\
31830_{\max } \\
33190_{\max } \\
34340 \\
44590_{\max } \\
37840_{\min } \\
41250 \\
42440 \\
49120_{\max } \\
50250\end{array}$ & $\begin{array}{l}8251012 \\
871_{\max } \\
927 \\
945 \\
955_{\max } \\
996_{\max } \\
1030 \\
1038_{\max } \\
1135_{\min } \\
1238 \\
1273 \\
1473_{\max } \\
1508\end{array}$ & $\begin{array}{c}4,3 \\
8,7_{\max } \\
6,4 \\
10,5 \\
21,7_{\max } \\
16,5_{\max } \\
9,4 \\
10,1_{\max } \\
1,8_{\min } \\
48 \\
90 \\
120 \max \\
362\end{array}$ \\
\hline
\end{tabular}

Le spectre se compose de deux bandes très distinctes d'intensilé beaucoup moins forte que celle du cétène correspondant.

L'une des bandes est complexe; elle présente une subdivision étroite.

Ces différentes bandes peuvent étre comparées à celle de l'acétone. Nous l'avons déjà fait pour l'ultra-violet dans la partie théorique de ce travail. Il est intéressant de voir quel rapport elles ont avec son spectre infra-rouge.

Victor Henri ( $\left.{ }^{2}\right)$ a trouvé que la fréquence du maximum d'une bande ultraviolette était dans un très grand nombre de cas à peu de chose près un multiple

(1) Le dimère du diéthylecétène a été préparé par M. Hooft d'après les indications de Staudinger et J. Mayer (B., 39, 1640 (1906) et Ann. (1913, p. 294).

(2) Victor Henri, Etudes de Photochimie, p. 161. 


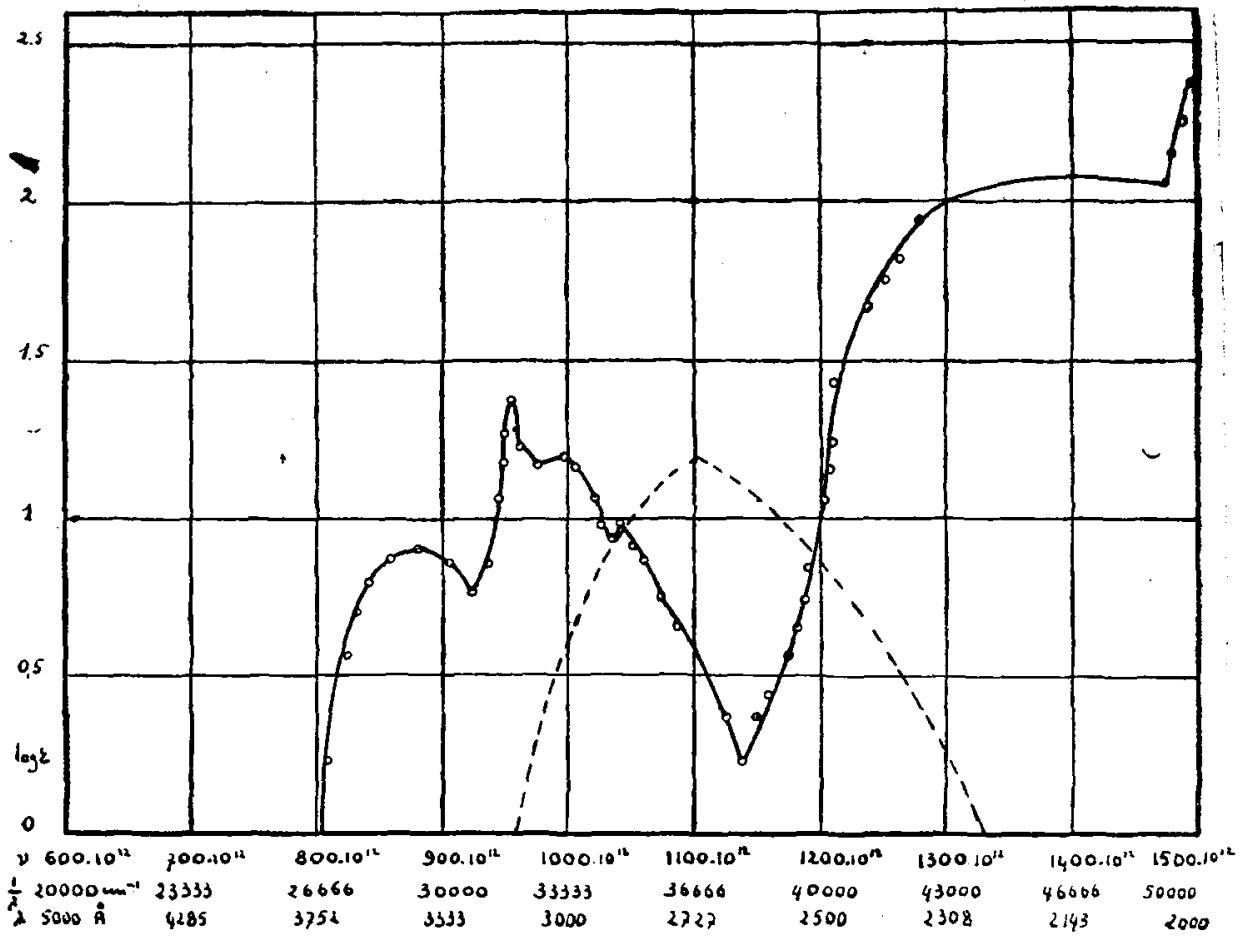

Fig. 6. - Spectro d'absorption da dimère du diéthylecétène (tétraéthyledicétocyclobutane)

$$
\begin{aligned}
& \left\langle\mathrm{C}_{2} \mathrm{H}_{5}\right)_{2} \mathrm{C}-\mathrm{C}=0 \\
& 0=\mathrm{C}-\mathrm{C}\left(\mathrm{C}_{2} \mathrm{H}_{5}\right)_{2}
\end{aligned}
$$

1. Trait plein : cn solution hexanique.

2. Trajt pointillé : Acétone en solution d'après Victor Henri. 
entier de la fréquence maximale d'une des bandes infra-rouges du même radical chimique.

$$
{ }_{{ }^{\mathrm{U}} . \mathrm{V} .}=r .{ }_{\text {I.R. }} \text { où } r \text { est un nombre entier ; }
$$

$r$ pour les dérivés de la fonction $=\mathrm{C}=\mathrm{O}$ est égal à 30 .

La première bande de notre dicétone possède 3 maxima distincts et un quatrième très peu prononcé. Le maximum le plus intense se trouve vers $\lambda=3142 \AA$ et $\nu=954.10^{12}$. Le second se trouve pour $\lambda=3013 \AA$ et $\nu=996.10^{12}$. Ces deux maxima sont en relation avec la bande infra-rouge $c^{\prime}$ de l'acétone dont la fréquence est $v=32,6.10^{12}\left({ }^{2}\right)$.

$$
30 \times 32,6.10^{12}=979.10^{12} \sim 975.10^{12}
$$

975.1012 est la moyenne arithmétique des fréquences des deux maxima $954.10^{12}$ et $996.10^{12}$.

Une même relation, dont la concordance est cependant moins bonne, existe entre le maximum de notre cyclobutane vers $\nu=871.10^{12}$ et la bande infra-rouge $b^{\prime}$ de l'acétone pour $\nu=27,5.10^{12}$.

$$
30 \times 27,5.10^{12}=825.10^{12}
$$

Enfin la deuxième bande avec une fréquence de $1373.10^{12}$ possède une relation avec une des bandes infra-rouges des hydrocarbures.

$$
1373.10^{12}=15 \times 91,5.10^{12}
$$

Ceux-ci ont une bande pour $\nu=\mathbf{8 9 . 1 0 ^ { 1 2 }}$, qui dans les alcools se déplace vers $\nu=91$ à $94.10^{12}$ et dans les cétones vers $\nu=92.10^{12}$.

\section{b) Le tétra-propyle-dicéto-cyclobutane.}

Ce corps a été préparé en maintenant du dipropylecétène pendant trois jours à $150^{\circ} \mathrm{C}$ en matras scellé $\left({ }^{2}\right)$. La purification a été faite par cristallisation dans l'hexane, où il se forme des paillettes. (P. F. $61^{\circ} \mathrm{C}$.)

$0,731 \mathrm{gr}$. ont été dissous dans $29,1 \mathrm{~cm}^{3}$ d'hexane. continue.

Nous avons photographié les spectres soit avec l'étincelle soit à la lumière

Les valeurs du coefficient d'absorption moléculaire pour les différentes longueurs d'ondes sont :

(1) Victor Henri, Etudes'de Photochimie, p. 32.

(2) Le travail de préparation et d'analyse a été fait par M. R. A. Bruschwerter selon les indications de Staudinger et Srrong (Hel $\omega$. Chim. Acta, VI, 296 (1923). 


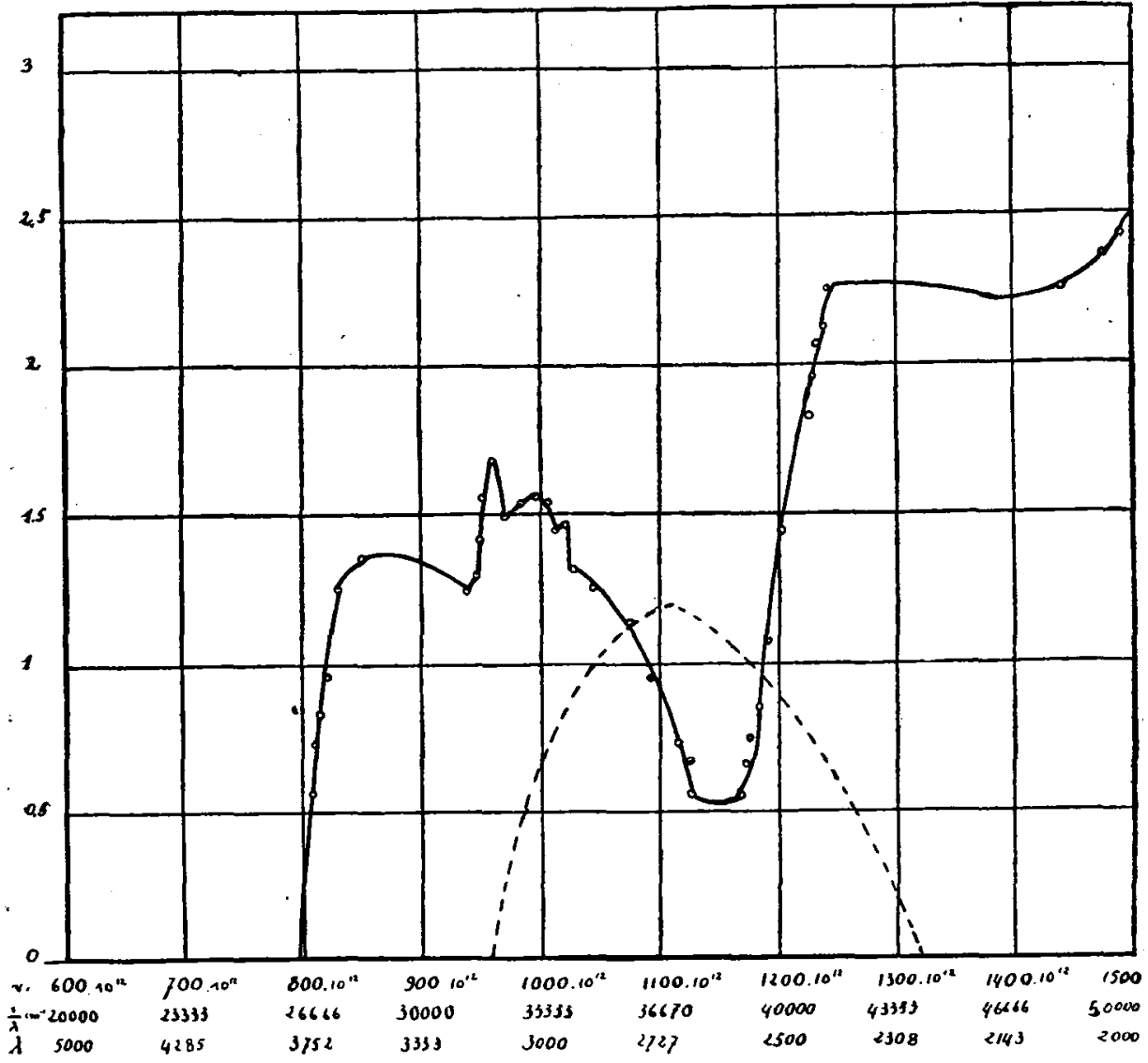

Fig. 7. - Spectre d'absorption du tétrapropyle-dicétocyclobutane

$$
\begin{aligned}
\left(\mathrm{C}_{3} \mathrm{H}_{7}\right)_{2} & =\mathrm{C}-\mathrm{C}=\mathbf{0} \\
\mathbf{0} & =\mathrm{C}-\mathrm{d}\left(\mathrm{C}_{3} \mathrm{H}_{7}\right)_{2}
\end{aligned}
$$

1. Trait plein : solution hexanique.

2. Trait pointillé : Acétone en eolution d'après Victor Henri. 


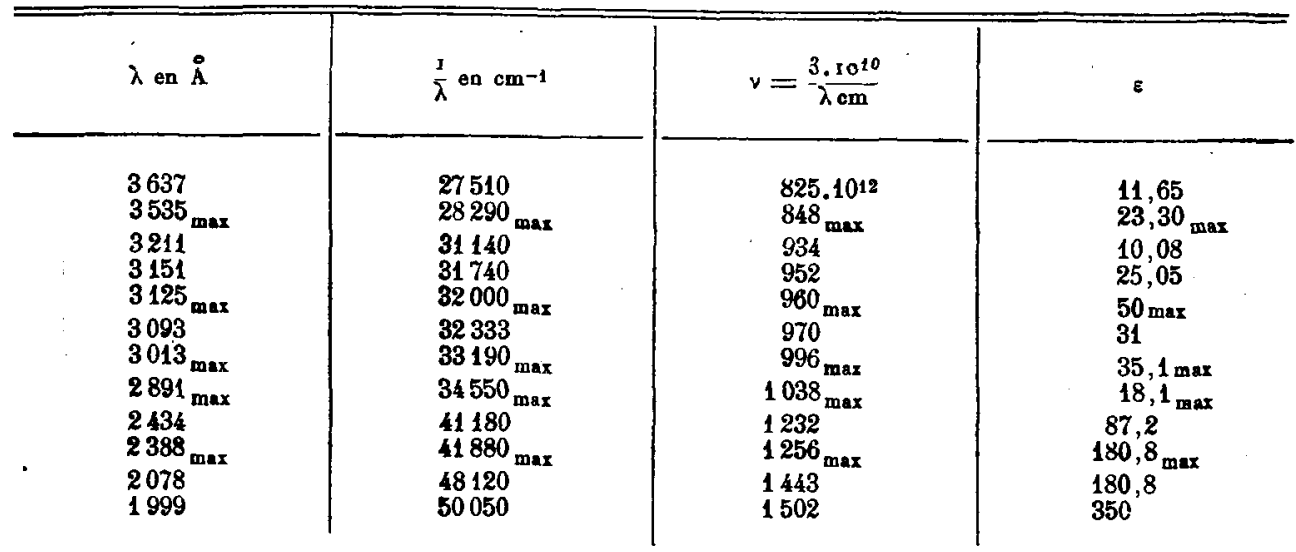

La courbe se compose comme celle du dérivé tétra-éthylé de 5 maxima, dont quatre forment une large bande.

Cette première bande peut être décomposée en deux bandes plus étroites :

L'une avec une valeur de $\varepsilon=23,3$ pour $\lambda=3535 \AA$ et $\nu=848.10^{12}$ correspond à la bande intra-rouge de l'acétone $b^{\prime}$

$$
27,5.10^{12} \times 30=825.10^{12}
$$

L'autre avec ses trois maxima pour :

$$
v=960.10^{12} \quad v=996.10^{12} \quad \text { et } v=1038.10^{12}
$$

est plus intense que celle du dimère du diéthylecétène. Elle lui est identique quant à la forme.

Le dernier maximum entre $\lambda=2388 \AA$ et $\lambda=2078 \AA$, vers $\nu=1350.10^{13}$ appartient à la deuxième bande. Il est dú à l'hydrocarbure. Son intensité doit provenir de la labilité du cycle formé par les quatre atomes de carbone.

Les spectres du tétra-éthyle et du tétra-propyle-dicéto-cyclobutane sont semblables. Ils ne diffèrent que par l'intensité de l'absorption. Celle-ci est presque trois fois plus forte pour les bandes des carbonyles et tend à devenir égale pour celle de l'hydrocarbure.

Dans la table suivante, nous donnons les rapports des coefficients d'absorption

\begin{tabular}{|c|c|c|c|c|c|c|}
\hline \multicolumn{3}{|c|}{ Tëtra-propylé } & \multicolumn{3}{|c|}{ Tétra-éthylo } & \multirow{2}{*}{$\begin{array}{c}\text { e propyló } \\
\text { E éthylé } \\
2,7 \\
2,68 \\
2,93 \\
2,43 \\
2,3 \\
2,15 \\
1,72 \\
1,82 \\
1,5 \\
0,97\end{array}$} \\
\hline $\begin{array}{c}\varepsilon=11,65 \\
23,3 \\
18,08 \\
25,5 \\
50 \\
31,1 \\
18,08 \\
87,2 \\
180,8 \\
350\end{array}$ & pour $\vee=$ & $\begin{array}{l}825.10^{2} \\
848 \\
934 \\
952 \\
960 \\
996 \\
1038 \\
1232 \\
1350 \\
1502\end{array}$ & $\begin{array}{r}\varepsilon=\quad 4,3 \\
8,7 \\
6,4 \\
10,5 \\
21,7 \\
16,5 \\
10,1 \\
48 \\
120 \\
362\end{array}$ & pour $\vee=$ & $\begin{array}{l}825.10^{1}= \\
871 \\
927 \\
945 \\
955 \\
996 \\
1038 \\
1238 \\
1373 \\
1508\end{array}$ & \\
\hline
\end{tabular}
des deux dérivés : 


\section{c) Lo tétra-allyle-dicétocy clobutane.}

Le diallylecétène $\left({ }^{1}\right)$, chauffé pendant 48 heures à $100^{\circ} \mathrm{C}$. en tube fermé, se décolore et produit une masse sirupeuse brunâtre.

Par distillation dans le vide on obtient trois fractions :

1) A $139^{\circ}$ pour $9 \mathrm{~mm} \mathrm{Hg}$ de $\mathrm{p}$

2). A $196^{\circ}$ pour $29^{\circ}$ pour $1 \mathrm{~mm}$ *

.................................

incolore

Ígèrement jaune

Nous n'avons pas obtenu assez de la seconde fraction pour pouvoir l'étudier. Les résultats de l'analyse élémentaire prouvent que c'est un polymère du cétène. Nous en ignorons le poids moléculaire $\left.{ }^{2}\right)$.

La première fraction par traitement à la soude caustique alcoolique donne de la tétra-allyle-acétone () (P. E. $122^{\circ}$ à $14 \mathrm{~mm} \mathrm{Hg} \mathrm{de} p$ ).

Pour la spectrographie, nous avons dissous $0,8151 \mathrm{gr}$. (P. E. $139^{\circ}$ à $9 \mathrm{~mm}$ de Hg) dans $33,41 \mathrm{~cm}^{2}$ d'hexane (sol. $\mathrm{mol} / 10$ ).

La table suivante donne la valeur de $\varepsilon$ pour les points principaux de la courbe d'absorption :

\begin{tabular}{|c|c|c|c|}
\hline$\lambda$ en $\AA$ & $\frac{1}{\lambda}$ en $\mathrm{cm}^{-1}$ & $v=\frac{3.10^{10}}{\lambda \mathrm{cm}}$ & $\varepsilon$ \\
\hline $\begin{array}{l}3750 \\
3600 \\
3447_{\max } \\
3234 \\
3196 \\
3151_{\text {max }} \\
3060 \\
3014_{\max } \\
2940_{\max } \\
2633_{\max } \\
2440 \\
2276 \\
2066\end{array}$ & $\begin{array}{l}26665 \\
27780 \\
29100_{\max } \\
30920 \\
31290 \\
31740_{\max } \\
31680 \\
33180_{\max } \\
34010_{\max } \\
37980_{\min } \\
40980 \\
43489 \\
48400\end{array}$ & $\begin{array}{l}800.1011 \\
833 \\
873_{\max } \\
927 \\
928 \\
952_{\max } \\
980 \\
996_{\max } \\
1020_{\max } \\
1139_{\min } \\
1229 \\
1318 \\
1452\end{array}$ & $\begin{array}{l}1,8 \\
11,6 \\
17,52_{\max } \\
14,2 \\
25 \\
51,5_{\max } \\
34,5 \\
40_{\max } \\
35_{\max } \\
53_{\min } \\
175 \\
1166 \\
2711\end{array}$ \\
\hline
\end{tabular}

La courbe est semblable a celle des deux autres dimères, pour la partio du spectre qui se trouve entre les fréquences $800.10^{12}$ et $1139.10^{12}$ où se trouvent les bandes de la fonction carbony lique.

(2) Staudinger et Schotz, Help. Chim. Acta, VI, p. 300.

(9) La préparation du diallylecétène et de son polymère a été faite par M. E. ZuBLIN.

Analyse élémentaire du produit 2) :

02890 grs de subst. donnent 0,8352 grs de $\mathrm{CO}^{2}$ et 0,2115 grs de $\mathrm{H}^{2} \mathrm{O}$ calculé pour $\left(\mathrm{C}^{8} \mathrm{H}^{10} \mathrm{O}\right)$ :

$\mathrm{C}=78,60 \% \mathrm{H}=8,20 \%$; trouvé : $\mathrm{C}=78,84 \%, \mathrm{H}=8,30 \%$.

(9) Analyse élémentaire de la tétra-allyle-acétone :

0,1794 grs de subst. donnent 0,5422 grs de $\mathrm{CO}_{2}$ et 0,1643 grs de $\mathrm{H}_{2} \mathrm{O}$;

0,1278 grs de subst. donnent 0,3857 grs de $\mathrm{CO}_{2}$ et 0,1181 grs de $\mathrm{H}_{2} \mathrm{O}$.

Calculé pour la tétra-allyle-acétone : $\mathrm{C}=82,49 \%$ et $\mathrm{H}=10,17 \%$.

Trouvé : $\mathrm{C}=82,44 \%$ et $\mathrm{H}=10,25 \% ; \mathrm{C}=82,35 \%$ et $\mathrm{H}=10,33 \%$. 
Dans l'ultra-violet extrême l'absorption est beaucoup plus forte que celle des dicéto-cyclobutanes saturés.

La première bande du spectre possède les quatre maxima suivants :

$$
\begin{array}{llll}
\lambda=3437 \AA ; & v=873.12^{12} & \text { et } & \varepsilon=17,52 \\
\lambda=3151 \AA ; & v=952 \text { n } & \text { et } & \varepsilon=51,5 \\
\lambda=3014 \AA ; & v=996 \text { ” } & \text { et } & \varepsilon=40 \\
\lambda=2940 \AA ; & v=1020 \text { » } & \text { et } & \varepsilon=35
\end{array}
$$

Ils sont moins prononcés que ceux du tétrapropyle-dicétocyclobutane et sont reliés au spectre infra-rouge de l'acétone par les mêmes relations que ceux-ci.

La deuxième bande très intense dans l'extrême ultra-violet n'est probablement pas à son maximum dans les régions que nous avons étudiées. Elle est plus forte que celle des dicétocyclobutanes saturés. C'est seulement par cette bande que le spectre du tétraallyledicétocyclobutane est différent de celui du tétrapropyledicétocyclobutane.

\begin{tabular}{|c|c|c|c|c|c|c|}
\hline \multicolumn{3}{|c|}{ Tátra-allylé } & \multicolumn{3}{|c|}{ Tétrapropylé } & $\frac{\varepsilon \text { allyle }}{\varepsilon \text { propyle }}$ \\
\hline$\varepsilon=\begin{array}{c}17,52 \\
14,2 \\
25 \\
51,5 \\
34,5 \\
40 \\
175 \\
2.711\end{array}$ & pour $\vee=$ & $\begin{array}{l}873.10^{12} \\
927 \\
938 \\
952 \\
980 \\
996 \\
1229 \\
1452\end{array}$ & $\varepsilon=\begin{array}{c}23,30 \\
18,08 \\
25,6 \\
50 \\
31 \\
35,1 \\
87,2 \\
180,8\end{array}$ & pour $v=$ & $\begin{array}{l}848.10^{12} \\
934 \\
952 \\
960 \\
970 \\
996 \\
1232 \\
1443\end{array}$ & $\begin{array}{c}0,752 \\
0,755 \\
0,98 \\
1,03 \\
1,11 \\
1,14 \\
2, * \\
15,05\end{array}$ \\
\hline
\end{tabular}

Victor Henri (') a déjà fait cette observation pour l'alcool propylique et l'alcool allylique, et pour l'acétone et la phorone.

Rapport entre l'intensité d'absor ption du tétrapropyle et du tétra-allyle-dicétocyclo-butane

Tandis qu'entre le tétra-éthyle et le tétrapropyle la valeur du rapport des intensités diminue avec la longueur d'onde, entre celui-ci et le tétra-allyle iI augmente avec la fréquence. Ceci est très caractéristique. Dans le premier cas nous avons l'effet de l'augmentation du radical hydrocarbure sur la fonction cétonique; dans le second cas l'action de quatre liaisons éthyléniques,

Si nous reportons la valeur du quotient $=15,05$ aux quatre liaisons éthyléniques nous obtenons un coefficient d'exaltation $=3,76$ pour chacune.

Celui observé pour l'alcool allylique est de 2,97 ( $\left.{ }^{2}\right)$.

d) Le diméthyle diphényle-dicéto-cyclobutane.

Dimère du phényle-méthyle-cétène il se prépare comme les dérivés alipha-

(1) Etudes de Photochimie, 1919 ; p. 103.

(2) Victor Henri, Etudes de Photochimie, p. 130. 


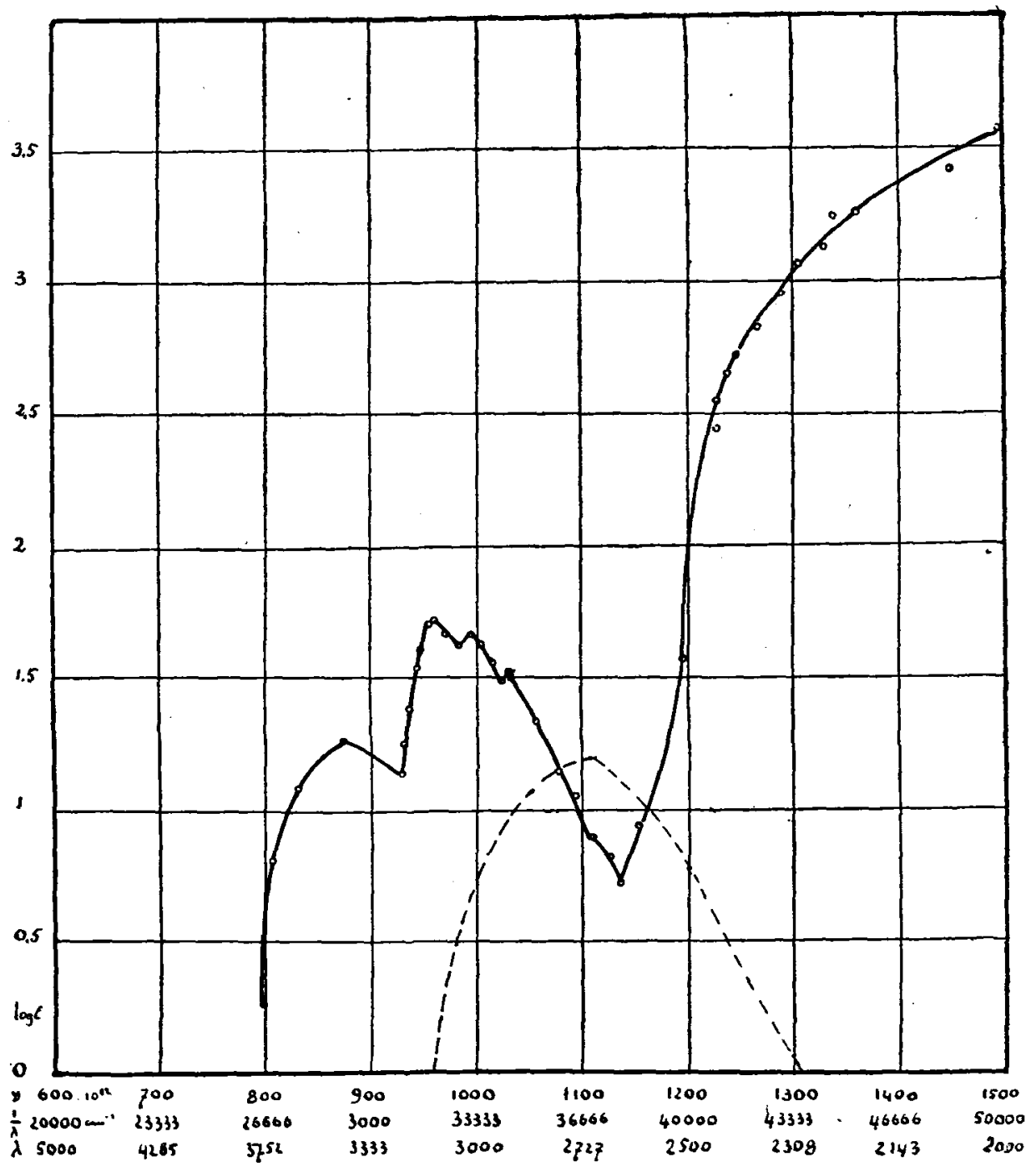

Fig. 8. - Spectre d'absorption du diallycótène dimère (tétra-allyledicétocyclobutane)

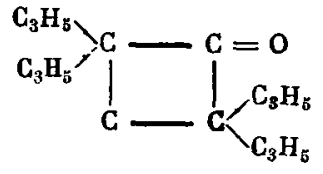

1. Trait plein : en solution hexanique.

2. Trait pointillé : Acétone d'après Vítor Henri. 
tiques par polymérisation à la chaleur $\left(^{1}\right)$. Nous l'avons cristallisé dans l'acétate d'éthyle et lavé à l'hexane pour le débarrasser des traces de ce solvant peu propre à la spectrographie des régions ultra-violettes.

Nous avons photographié les spectres d'étincellẹs et continus des solutions $2 / 3 \mathrm{~mol} / 1000$ (1, $2 / 3 \mathrm{~mol} / 1100$ et $1,2 \mathrm{~mol} / 10$.

Les points que nous avions coutume de mesurer au moyen de la concentration mol/100 l'ont été cette fois au moyen des grandes épaisseurs (10 à 100 millimètres) de la solution $2 / 3 \mathrm{~mol} / \mathbf{1 0 0 0}$.

La solution 1/2 mol/10 n'a pu être photographiée que dans l'acétate de méthyle pur. Les autres solvants sont tous saturés pour des concentrations moins fortes.

$0,1898 \mathrm{gr}$. (P. F. 161,5-162 non corr.) ont été dissous dans $14,4 \mathrm{~cm}^{2}$ d'acétate de méthyle $(1 / 2 \mathrm{~mol} / 10)$.

$0,0066 \mathrm{gr}$. ont été dissous dans $38,1 \mathrm{~cm}^{8}$ d'alcool éthylique (sol : $0,68 \mathrm{~mol} / \mathbf{1 0 0 0})$.

Les valeurs de $\varepsilon$ pour les différentes longueurs d'ondes sont les suivantes.:

\begin{tabular}{|c|c|c|c|}
\hline$\lambda=$ on $\AA$ & $\frac{\mathrm{r}}{\lambda}$ en $\mathrm{cm}^{-1}$ & $v=\frac{3.10^{10}}{\lambda \mathrm{cm}}$ & $\varepsilon$ \\
\hline $\begin{array}{l}3824 \\
3735 \\
3645 \\
3618 \\
3470_{\max } \\
3250 \\
3177 \\
3180_{\max } \\
3415_{\max } \\
2934_{\max } \\
2853_{\min } \\
2779 \\
2737 \\
2705 \\
2658 \\
2599 \\
2461 \\
2370 \\
2294 \\
2210 \\
2112\end{array}$ & $\begin{array}{l}26150 \\
26770 \\
27480 \\
26640 \\
28820_{\max } \\
30780 \\
31480 \\
31450_{\max } \\
33170_{\max } \\
34080_{\max } \\
35050_{\operatorname{mix}} \\
35980 \\
36670 \\
36970 \\
37620 \\
38480 \\
40630 \\
42190 \\
43590 \\
45250 \\
47350\end{array}$ & $\begin{array}{l}785.1012 \\
803 \\
823 \\
829 \\
865_{\max } \\
923 \\
943 \\
944_{\max } \\
996_{\max } \\
1023_{\max } \\
1052_{\min } \\
1080 \\
1100 \\
1109 \\
1129 \\
1154 \\
1219 \\
1266 \\
1308 \\
1357 \\
1420\end{array}$ & $\begin{array}{c}1,8 \\
17,5 \\
54 \\
70,3 \\
105_{\max } \\
54,2 \\
131 \\
145_{\max } \\
143_{\max } \\
130_{\max } \\
54,2_{\operatorname{mix}} \\
54,2 \\
203 \\
350 \\
543 \\
1050 \\
2715 \\
5285 \\
8140 \\
26300 \\
40700\end{array}$ \\
\hline
\end{tabular}

La courbe d'absorption est la même que celle des autres $\beta$-dicétones non énolisables. La bande des carbonyles est plus intense ce qui est dû à la présence des radicaux aromatiques. Ceux-ci ne sont pas caractérisés par une ou plusieurs petites bandes. Nous avons pu faire la mème observation pour le diphénylecétène. La position des bandes est légèrement déplacée.

(1) Ce corps a été préparé par M. Haendy selon les données de Staudisger et L. Ruzicka, Ann., $380 ; 278$ (1911). 


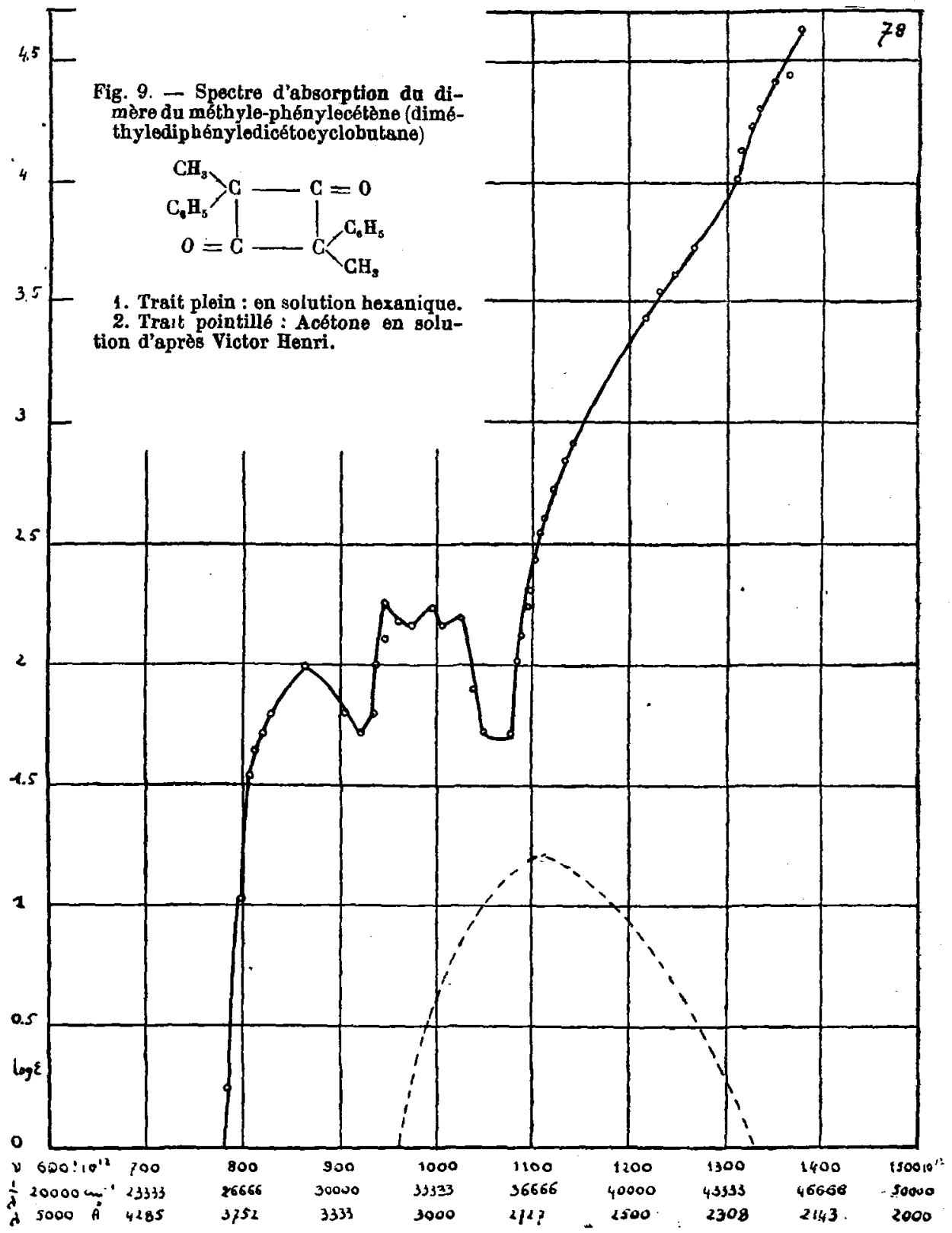


La première bande est formée de quatre maxima :

Le premier se trouve vers

$$
\lambda=3470 \AA \quad \nu=865.10^{12} \quad \text { son intensité est } \quad \varepsilon=105
$$

Les trois autres pas très distincts se trouvent vers

$$
\begin{array}{llll}
\lambda=3180 \AA & \nu=944.10^{12} & \text { et } & \varepsilon=145 \\
\lambda=3015 \AA & \nu=996.10^{12} & \text { et } & \varepsilon=143 \\
\lambda=2934 \AA & v=102310^{12} & \text { et } & \varepsilon=130
\end{array}
$$

L'absorption de l'ultra-violet extrême correspond par son intensité à celle. des groupes phényles.

\section{5. - SPECTRES D'ABSORPTION DES DIMERES DES CETENES, AVEC FONCTION ÉNOLIQUE POSSIBLE}

\section{a) Le dicétocyclobutane.}

Le cétène dimère s'obtient en abandonnant quelques jours du cétène sous pression en matras scellé à la température ordinaire.

Il se forme un liquide brun qui bout entre $126^{\circ}$ et $127^{\circ} \mathrm{C}$ à pression ordinaire et par rectification devient incolore $\left({ }^{1}\right)$.

Nous sommes parvenu à le cristalliser en plongeant sa solution hexanique dans un mélange réfrigérant de neige de $\mathrm{CO}_{2}$ - éther.

Le produit ainsi purifié est parfaitement incolore, mais n'est pas stable à la température ordinaire. Il se décompose et se colore en jaune dans l'espace de quelques heures (environ 6 heures).

Nous avons photographié le spectre en solution éthérée et en solution hexanique. Nous n'avons pas essayé de le photographier en solution alcoolique car une décomposition était très probable.

Les ampoules qui contenaient le dicétocyclobutane n'ont pas séjourné plus d'une nuit avant d'être spectrographiées. Ėlles plongeaient dans un mélange réfrigérant, aussi le corps était-il encore incolore au moment où nous avons préparé les solutions.

La courbe d'absorption si elle ne possède pas de oandes aussi distinctes que les dérivés aliphatiques, est cependant caractéristique et non pas une "small général absorption " comme celle qu'a mesurée Watson ( $\left.{ }^{2}\right)$.

$0,2120 \mathrm{gr}$. (P. E. $126^{\circ}-127^{\circ}$ à p. normale) ont été dissous dans $25,24 \mathrm{~cm}^{*}$ d'éther (sol. mol/10).

$0,2875 \mathrm{gr}$. ont été dissous dans $34,22 \mathrm{~cm}^{2}$ d'hexane (sol. mol/10).

(1) Chick et Wilsmore, Jour. Chem. Soc., 93, 946 (1908), et Staudinger, Les Cétènes, p. 41.
(2) Jour. Chem. Soc., 93, 946 (1908). 
Voici qu'elles sont les valeurs du coefficient d'absoption moléculaire :

10 Sclution éthérée :

\begin{tabular}{|c|c|c|c|}
\hline$\lambda$ en $\stackrel{\AA}{A}$ & $\frac{\mathrm{I}}{\pi}$ en $\mathrm{cm}-1$ & $v=\frac{3 \cdot 10^{10}}{\lambda \mathrm{cm}}$ & $\varepsilon$ \\
\hline $\begin{array}{l}3369 \\
3277 \\
3177 \\
3119 \\
3113_{\max } \\
3083 \\
2994 \\
2980 \\
2858 \\
2684_{\max } \\
2506 \\
2351_{\max } \\
2210 \\
2135 \\
2105\end{array}$ & $\begin{array}{l}29680 \\
30520 \\
31480 \\
\left\{\begin{array}{l}32060 \\
32120 \\
32440 \\
33400\end{array}\right. \\
33560 \\
34990 \\
37260_{\text {max }} \\
40110 \\
42540_{\max } \\
45250 \\
46830 \\
47510\end{array}$ & $\begin{array}{l}891.10^{12} \\
915 \\
944 \\
961 \\
964 \\
973 \\
1002 \\
1007 \\
1049 \\
1117_{\max } \\
1203 \\
1276_{\max } \\
1357 \\
1405 \\
1426\end{array}$ & $\begin{array}{c}1.15 \\
4,67 \\
6.7 \\
9,7 \\
9.7 \\
9,7 \\
9,91 \\
11,5 \\
23,4 \\
27.12_{\max } \\
87,5 \\
135.5_{\max } \\
180,8 \\
360,13 \\
540,19\end{array}$ \\
\hline
\end{tabular}

2o Solution hexanique :

\begin{tabular}{|c|c|c|c|}
\hline$\lambda$ en $\AA$ & $\frac{1}{\lambda}$ en $\mathrm{cm}^{-1}$ & $\nu=\frac{3 \cdot \mathrm{ro}^{10}}{\lambda \mathrm{cm}}$ & $\varepsilon$ \\
\hline $\begin{array}{l}3261 \\
3151 \\
(3133 \\
3116_{\max } \\
3080 \\
3019 \\
2970 \\
2944 \\
2857 \\
2684_{\max } \\
2499 \\
2344_{\text {max }} \\
2255 \\
2117 \\
1999\end{array}$ & $\begin{array}{l}30670 \\
31740 \\
31330 \\
32040 \text { max } \\
32470 \\
33120 \\
33670 \\
33970 \\
35000 \\
37260_{\max } \\
40500 \\
42666_{\max } \\
49350 \\
\mathbf{4 7 2 4 0} \\
56020\end{array}$ & $\begin{array}{l}920.10^{12} \\
952 \\
\{957 \\
963_{\max } \\
974 \\
993 \\
1010 \\
1019 \\
1050 \\
1117_{\max } \\
1215 \\
1279_{\max } \\
133: \\
1471 \\
1502\end{array}$ & $\begin{array}{c}4,6 \\
6,7 \\
\{6,7 \\
6,7_{\max } \\
6.7 \\
9,7 \\
9,9 \\
11,5 \\
23,2 \\
27,12_{\max } \\
87,5 \\
90,31_{\max } \\
116,6 \\
467 \\
1355\end{array}$ \\
\hline
\end{tabular}

La courbe se compose de trois maxima dont l'intensité diffère selon le solvant.

Les deux premiers qui forment une première bande correspondent aux bandes infra -rouge de l'acétone $c^{\prime}$ et $a$.

Ils se trouvent aux longueurs d'ondes suivantes :

$1^{\circ} \lambda=3083 \AA$ et $\nu=9.3 .10^{12}$ et $\varepsilon=9,7\left(^{1}\right) ; 30 \times 32,6.10^{12}=978.10^{12}$ $\nu=32,6.10^{12}$ est la fréquence de la bande $c^{\prime}$ de l'acétone.

(1) $\varepsilon$ en solution hexanique est légèrement inférieur il est égal à 6,7 . 


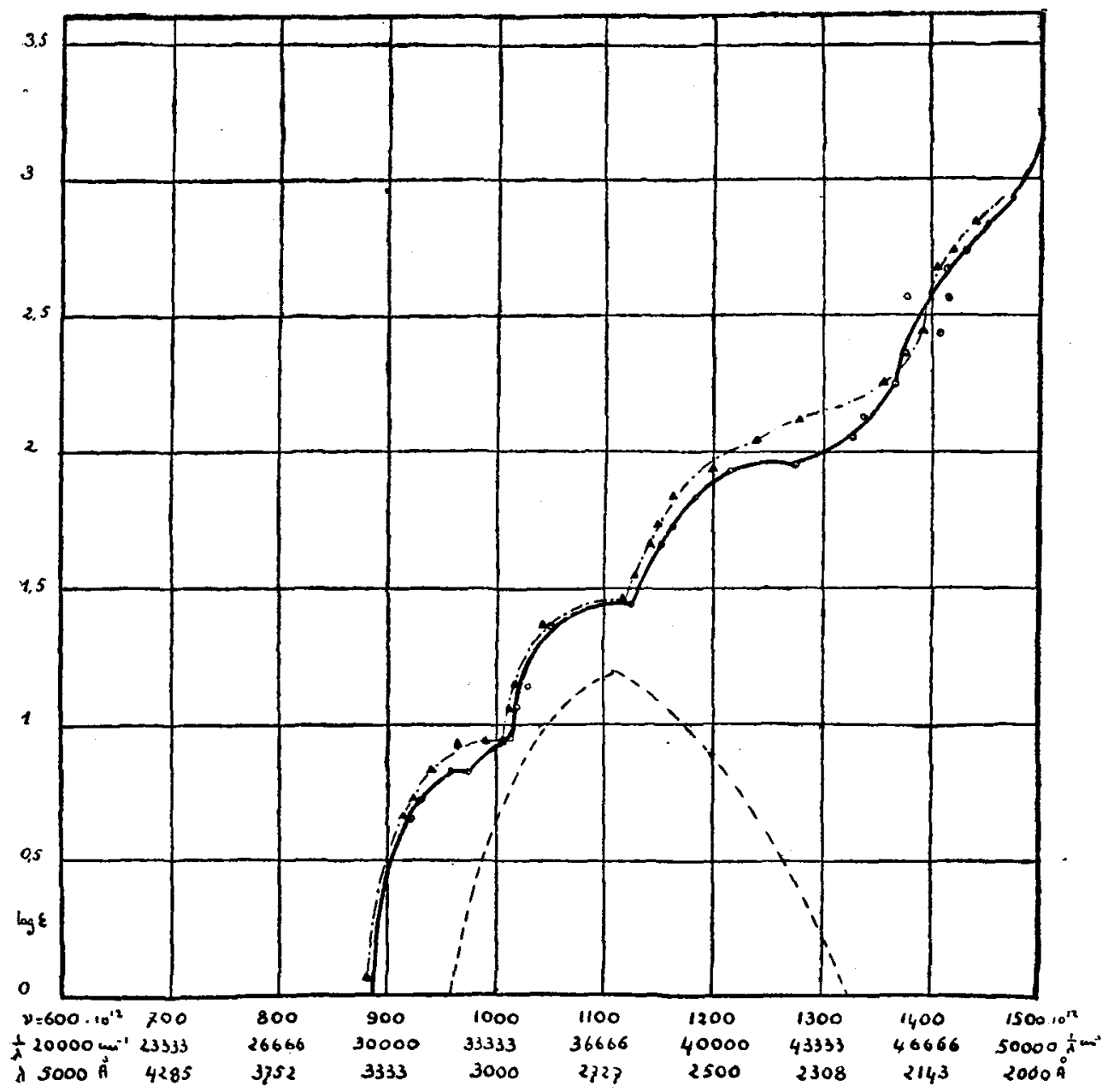

Fig. 10. - Spectre d'absorption du dimère du cétène, le dicétocyclobutane

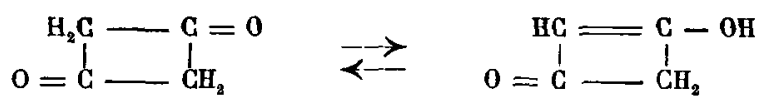

1. En trait plein : en solution hexanique.

$\boldsymbol{z}$. En trait - - - - : en solution éthèrée.

3. En trait - - - - - : Acétone d'après Victor Hebri. 
$2^{\circ} \lambda=2684 \AA$ et $\nu=1117.10^{12}$ et $\varepsilon 27,12 ; 30 \times 36,5.10^{12}=1096.10^{12}$ $\nu=36,5$ est la fréquence de la bánde infra-rouge $a$ de l'acétone $\left(^{\mathbf{x}}\right)$.

Le troisième maximum se trouve pour une tréquence $\nu=1276,10^{12}$ et un $\varepsilon=136$ en solution éthérée. En solution hexanique il semble se déplacer vers l'ultra-violet extrême et son intensité est moins grande $\nu_{\max }=1279,10^{12}$ et $\varepsilon_{\max }=90,3$. Il a la même position pour le diméthyledicétocyclobutane (forme énolique) ( $\left.{ }^{2}\right)$.

\section{b) Le diphényle-dicétocyclobutane :}

Staudinger et Bereza ont obtenu ce corps en essayant de préparer le monophénylecétène. Ils ont traité en solution éthérée le chlorure de l'acide phénylacétique chloré avec des rognures de zinc $\left(^{3}\right)$.

Les produits de réaction sont séparés du chlorure de zinc qui précipite par addition de benzine. Après lavage à l'eau, à l'acide $\mathrm{HCl}$ et à la soude, on obtient : $1^{\circ}$ une masse résineuse brune; $2^{\circ}$ dans la solution alcaline la forme énolique et $3^{\circ}$ dans la benzine la forme cétonique du diphényledicétocyclobutane.

Celle-ci cristallise par évaporation à froid du solvant. Ce n'est qu'après plusieurs cristallisations dans l'hexane, que nous avons obtenu le dérivé cétonique pur (F. P. $71, \mathfrak{b}^{\circ}-72,5^{\circ} \mathrm{C}$.).

La forme énolique est plus délicate à isoler. Nous avons repris tous les résidus de cristallisation de la dicétone avec de la soude caustique deux fois normale. Ils s'y dissolvent avec un faible dégagement de chaleur et en précipitent lorsqu'on aciditie, sous forme de flocons blancs. Nous les avons dissous dans de l'acétate d'éthyle absolu bouillant, auquel on ajoute une quantité égale de benzène absolu. On laisse refroidir et évaporer lentement dans le vide. Il se forme un dépót cristallin blanc qui est la forme énolique du diphényle-dicétocyclobutane (P. F. avec décomposition, $159-160^{\circ} \mathrm{C}$.)

Nous avons essayé, de remplacer le benzène par de l'hexane à cause de la spectrographie. Mais nous n'avans pas obtenu de bons résultats. Le produit précipité de sa solution dans l'acétate d'éthyle par de la benzine se décomposait déjà à $137^{\circ}-140^{\circ}$ et se colorait en jaune-brun, lorsque nous l'abandonnions dans un exsiccateur. Nous nous sommes donc contentés de laver les cristaux provenant de la solution benzénique avec de l'hexane bouillant pendant quelques heures.

\section{Spectrographie de la forme cétonique :}

Le spectre a élé mesuré en solution hexanique et alcoolique soit avec l'étincelle soit à la lumière continue.

(1) Comme pour les autres cyclobutanes, nous donnons ces relations avec les spectres infrarouges de l'acétone à titre de document, comme l'a fait Victor Henri dans son Etude de Photochimie.

(2) Voir spectre du diméthyle-dicétocyclobutane, p. 73.

- (3) B., 44; 537 (1911), et Thèse de BÉreza. 
I) Solution hexanique :

$0,2130 \mathrm{gr}$. (P. F. $71,5 \cdot 72,5^{\circ} \mathrm{G}$.) ont été dissous dans $18,1 \mathrm{~cm}^{3}$ d'hexane (sol. $1 / 2 \mathrm{~mol} / 10)$.

$0,0356 \mathrm{gr}$. ont été dissous dans $3,02 \mathrm{~cm}^{3}$ d'hexane (sol. $1 / 2 \mathrm{~mol} / 10$ ).

Les valeurs du coefficient d'absorption moléculaire, pour les différentes longueurs d'ondes sont les suivantes :

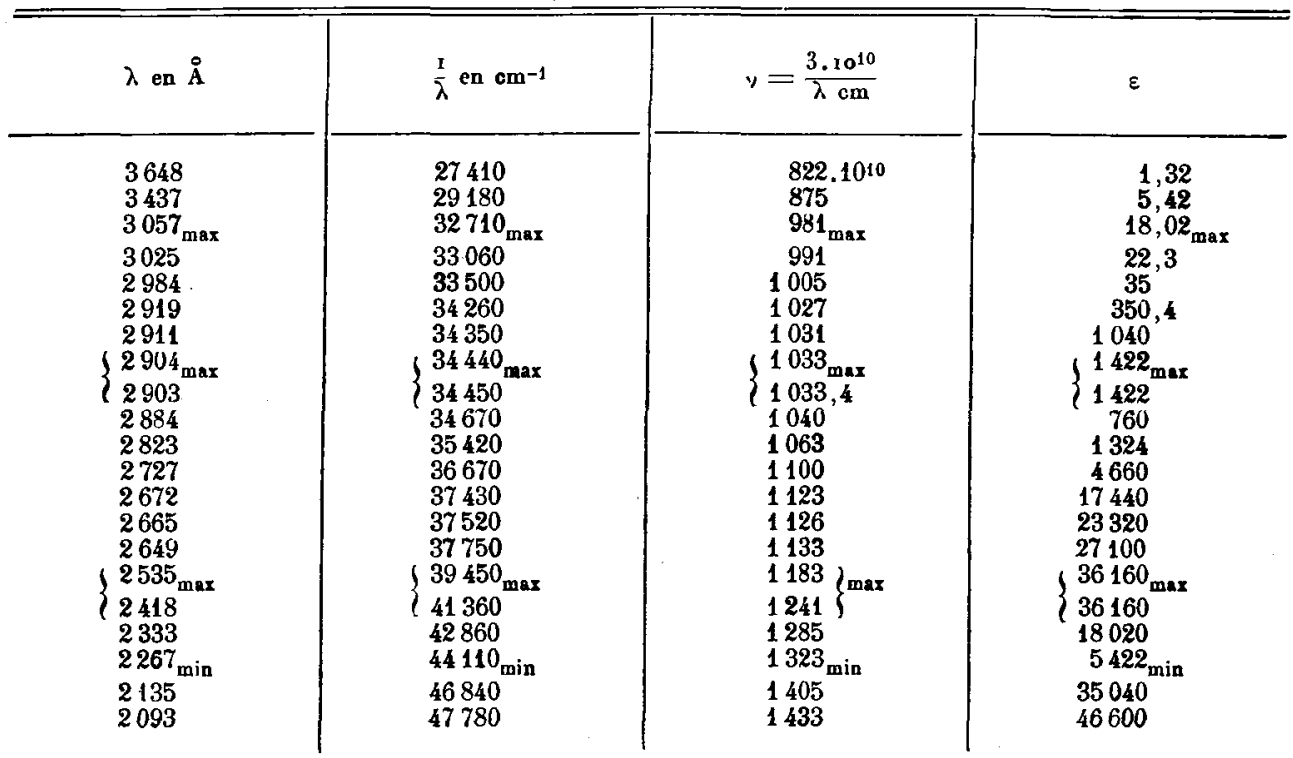

La courbe d'absorption peut se décomposer en deux régions, l'une s'étend de l'ultra-violet extrême à la fréquence $\nu=1000.10^{12}$, l'autre de cette fréquence au visible.

Dans la première nous avons le type d'absorption des dérivés aromatiques substitués.

$1^{\circ}$ Une bande fine :

\begin{tabular}{l|c|c}
\hline Notre corps & Isocyanate de phényle & Phẻnol (1) \\
\hline $\begin{array}{l}\nu=1033.10^{12} \\
\varepsilon=1442\end{array}$ & $\begin{array}{l}\nu=1082.10^{12} \\
\varepsilon=440^{2}\end{array}$ & $\begin{array}{l}\nu=1082.10^{22} \\
\varepsilon=2400\end{array}$
\end{tabular}

$2^{\circ}$ Une bande large :

\begin{tabular}{|c|c|c|}
\hline Notre corps de & Isocyanate de phényle de & Diphénylecétène do \\
\hline $\begin{array}{c}\nu=1183 \text { à } 1240.10^{12} \\
\Delta v=57.10^{12} \\
\varepsilon=36160\end{array}$ & $\begin{aligned} v=1302 \mathrm{a} 1335.10^{12} \\
\Delta v=33,10^{12} \\
\varepsilon=11600\end{aligned}$ & $\begin{array}{c}\nu=1109 \text { à } 1199 \cdot 10^{12} \\
\Delta \nu=90,10^{12} \\
\varepsilon=23350\end{array}$ \\
\hline
\end{tabular}

(1) F. W. Klingstedt, C. R., t. 174, p. 812 (1922). 

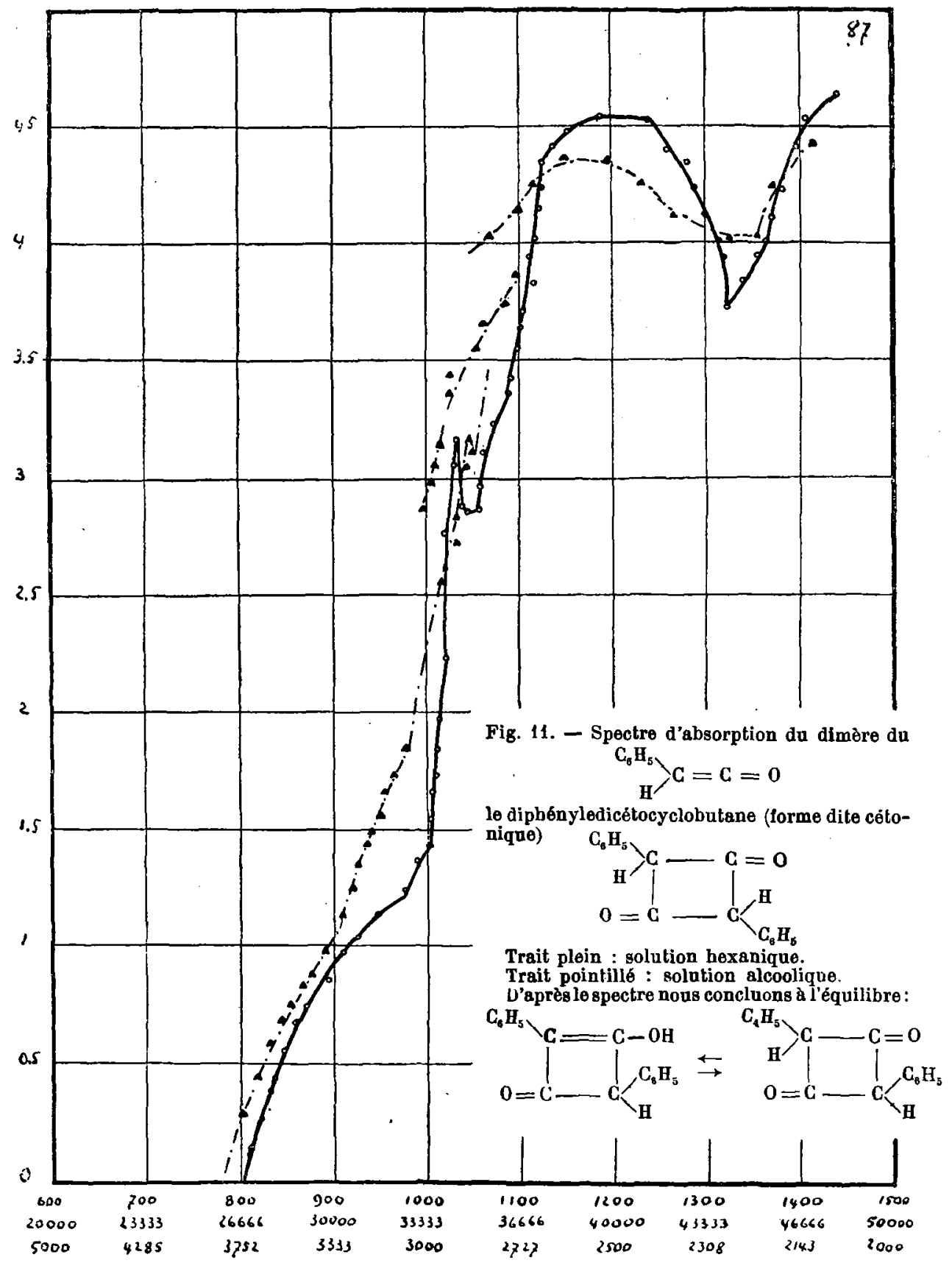

Guillaume C. Lardy. 
Dans l'autre région nous trouvons l'indication d'une bande dont le sommet pourrait être vers $\nu=981.10^{12}$. Elle correspondrait à la bande caractéristique des dicétones non énolisables et à la bande infra-rouge $c$ de l'acétone.

II) Solution alcoolique :

$0,1626 \mathrm{gr}$. (P. F. $71,5-72,5^{\circ} \mathrm{C}$.) sont dissous dans $13,7 \mathrm{~cm}^{2}$ d'alcool éthylique (sol. 1/2 mol/10).

Nous avons mesuré les valeurs suivantes pour le coefficient d'absorption :

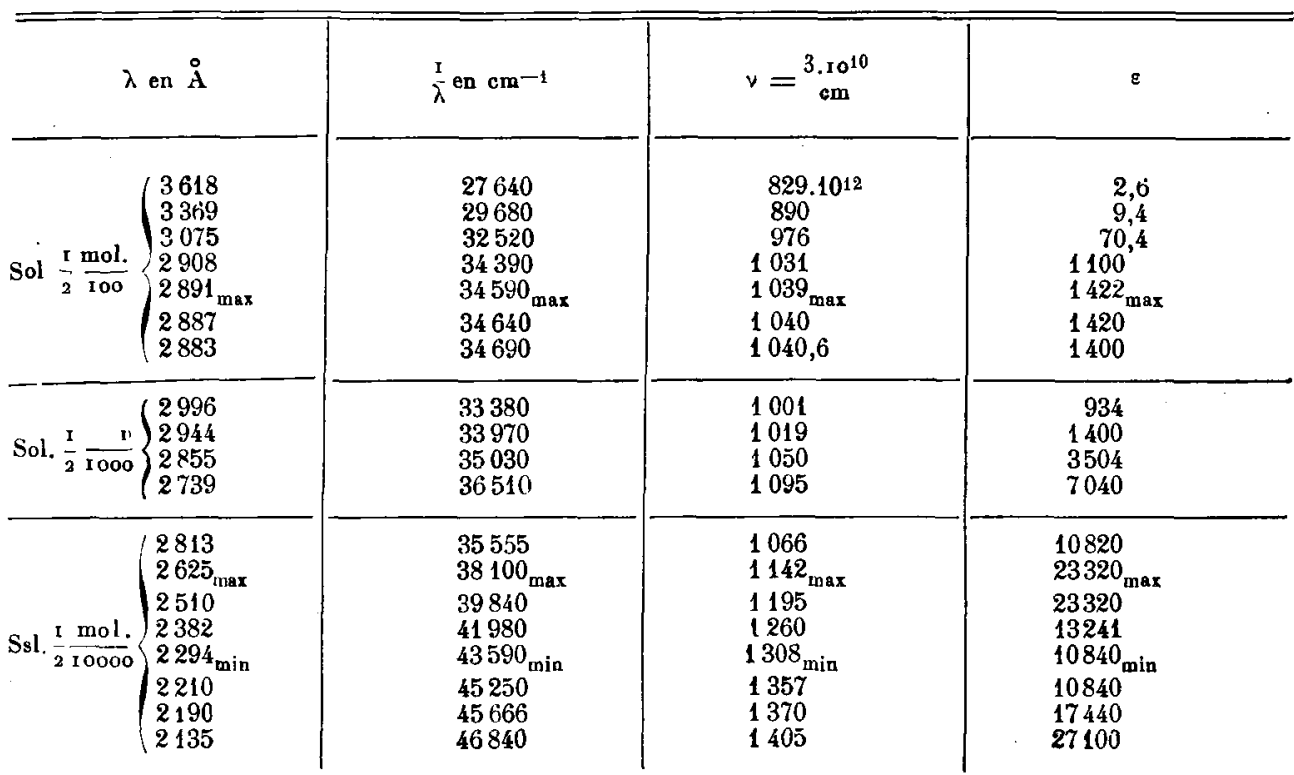

Nous retrouvons dans le spectre de la solution alcoolique les mèmes caractères que dans celui de la solution hexanique. La molécule du diphényledicétocyclobutane n'est cependant pas dans le même état.

$1^{\circ}$ La bande fine (aromatique) est déplacée vers l'ultra-violet.

$$
\left.\begin{array}{l}
v_{\max }=\text { solution hexanique } 1033.12^{12} \\
v_{\max }=\text { solution alcoolique } 1039.10^{12}
\end{array}\right\} \Delta^{v}=+6.10^{12}
$$

La bande intense et large est déplacée vers le rouge et son intensité est moins forte.

$$
\begin{gathered}
\text { Solution hexanigue } \\
v_{\max }=118 \overline{3.1} 0^{12} \text { à } 1241.10 \\
\varepsilon=36160
\end{gathered}
$$$$
\begin{gathered}
\text { Solution alcoolique } \\
\begin{aligned}
\nu_{\max } & =1143.10^{12} \\
\varepsilon & \text { à } 1195.10^{12}
\end{aligned} \\
=23320
\end{gathered}
$$

Le minimum pour $\nu=1323.10^{12}$ n'est plus aussi prononcé dans la solution alcoolique. 
$2^{\circ}$ Pour des fréquences inférieures à $1000.10^{12}$ toute l'absorption est déplacée vers le visible. La bande dont nous avons supposé le sommet pour $\nu=978.10^{12}$ n'est plus que très faiblement indiquée par une inflexion de la courbe vers $\nu=890.10^{12}$ environ.

En certains endroits la courbe présente une discontinuité. Pour des mêmes valeur de $\nu$ nous trouvons deux valeurs du coefficient d'absorption moléculaire. Ces défauts de concordance se trouvent aux endroits où l'absorption a été mesurée au moyen de deux solutions de concentrations différentes, sous des épaisseurs telles, que les quantités de substance interposées étaient équivalentes. Nous en concluons que la molécule a subi des transformations selon les concentrations. Nous supposons que l'équilibre entre la forme énolique et la forme cétonique varie, celle-ci étant en moins grande concentration par rapport à la forme énolique dans les grandes dilutions.

Nous pouvons aussi supposer que sous l'influence du rayonnement ultraviolet la forme labile cétonique se transforme en la forme stable énolique. Nous ne pouvons affirmer la chose; le fait important est qu'une transformation a lieu. Ceci prouve l'hypothèse de W. Dieckmann sur la possibilité d'un équilibre ( $\left.{ }^{2}\right)$.

\section{Spectrographie de la forme énolique:}

D'après les résultats obtenus par Victor Henri pour l'acétylacétate d'éthyle et autres composés énoliques $\left({ }^{2}\right)$, nous pouvions nous attendre à trouver la différence des spectres des deux tormes tautomères du diphényledicétocyclobutane entre les fréquenı'es $\nu=1100.10^{12}$ et $\nu=1300,10^{12}$, pour une très forte intensité.

Il ne nous a pas été possible d'étudier la forme énolique du diphényledicétocyclobutane aux grandes concentrations car il est très peu soluble dans les solvants usuels de la spectrographie comme l'éther; dans l'alcool il se décompose, dans l'hexane il ne se dissout pas. Nous n'avons pas disposé de quantités suffisantes de substance pure pour en photographier le spectre en solution alcaline.

\section{Solution éthérée :}

$0,0603 \mathrm{gr}$. (P. F. 160 $)$ ont été dissous dans $38 \mathrm{~cm}^{3}$ d'éther (sol. $0,66 \mathrm{~mol} / 100$ ).

A cette concentration la dissolution n'a pas été complète. Pour avoir les concentrations plus faibles nous avons dilué des quantités déterminées de suspension après avoir fortement agité.

Les valeurs trouvées sont donc plutôt trop faibles. Nous avons pu nous rendre compte que la correction à apporter est petite. La bande étroite des noyaux aromatiques, qui apparâ̂t très faiblement (trop faiblement pour être mesurée au photomètre) a pu être utilisée comme repère. Les solutions $0,66 \mathrm{~mol} / 1000$ et 0,66 $\mathrm{mol} / 10000$ étaient claires.

(1) W. Dieckmann et A. Wittmany, $B ., 55$, p. 3340 (1922).

(2) Victor Henri, C. R., t. 155, p. 1617 (1912). 
Les valeurs de $\varepsilon$ sont les suivantes :

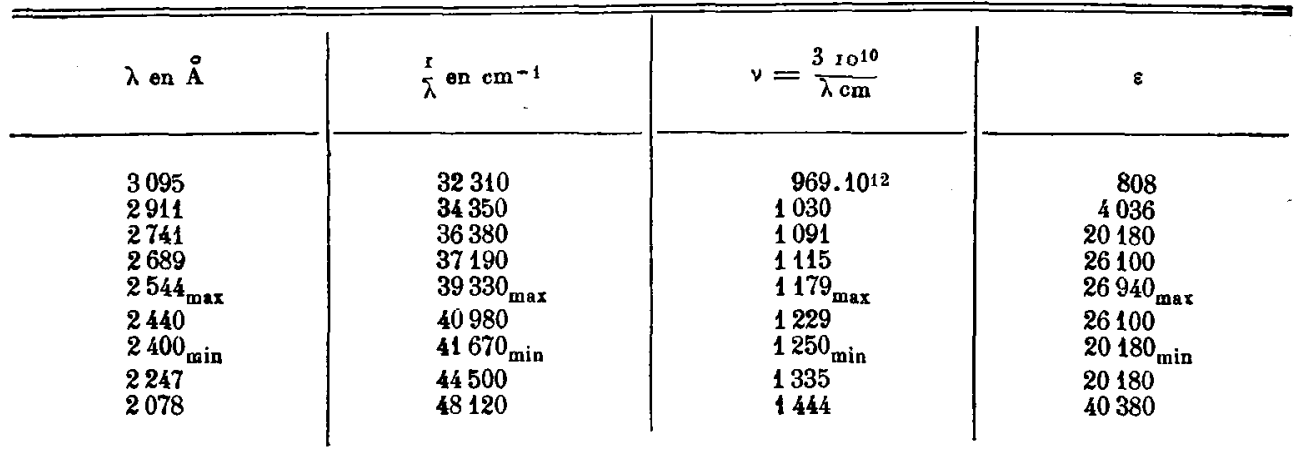

La courbe d'absorption se compose (pour la région ultra-violette extrême) d'une large bande. Comparée à celle de la forme dite cétonique, elle est déplacée vers les plus grandes longueurs d'onde. Le minimum, qui la suit, est très peu prononcé.

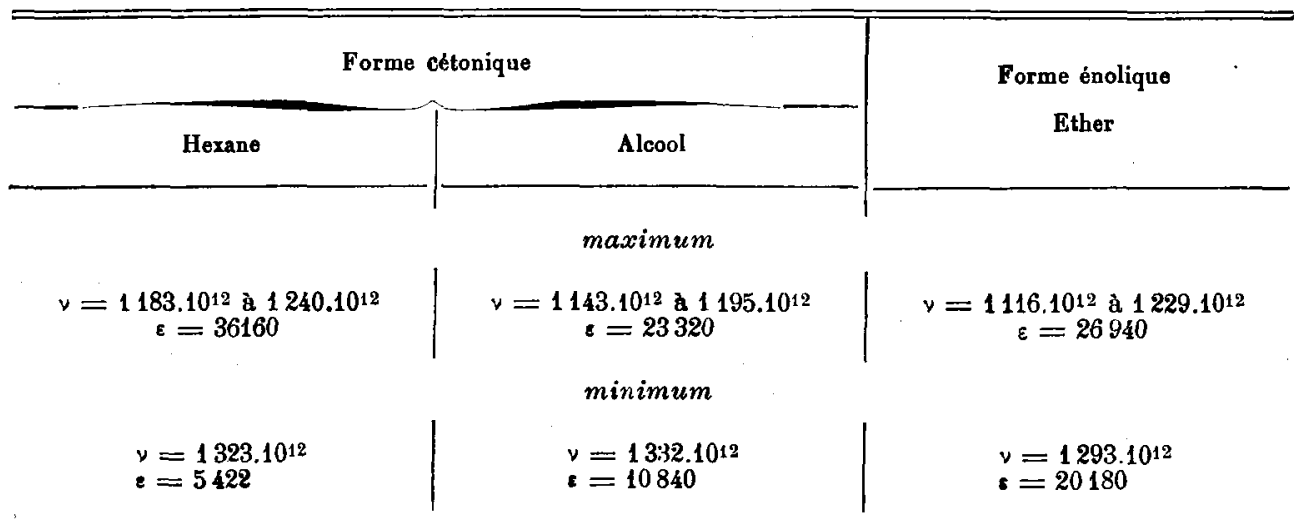

L'analogie très grande, aussi bien par la forme des courbes que par leur intensité, entre les spectres du diphényledicétocyclobutane dans les trois solutions montre que les deux formes tautomères peuvent se trouver en équilibre. Le spectre d'absorption du diphényledicétocyclobutane en solution hexanique diffère totalement de celui du diméthyle-diphényle-dicétocyclobutane (voir page 59).

c) Ether diéthylique de l'acide 1-3 dicarbonique du 1-3 diméthyle 2-4 dicétocyclobutane.

En chauffant dans du xylène l'ẹther éthylique de l'acide diazoacétylacétique :

$$
\mathrm{CH}_{3} \mathrm{CO} \mathrm{C}\left(\mathrm{N}_{2}\right) \mathrm{COOC}_{2} \mathrm{H}_{5}
$$




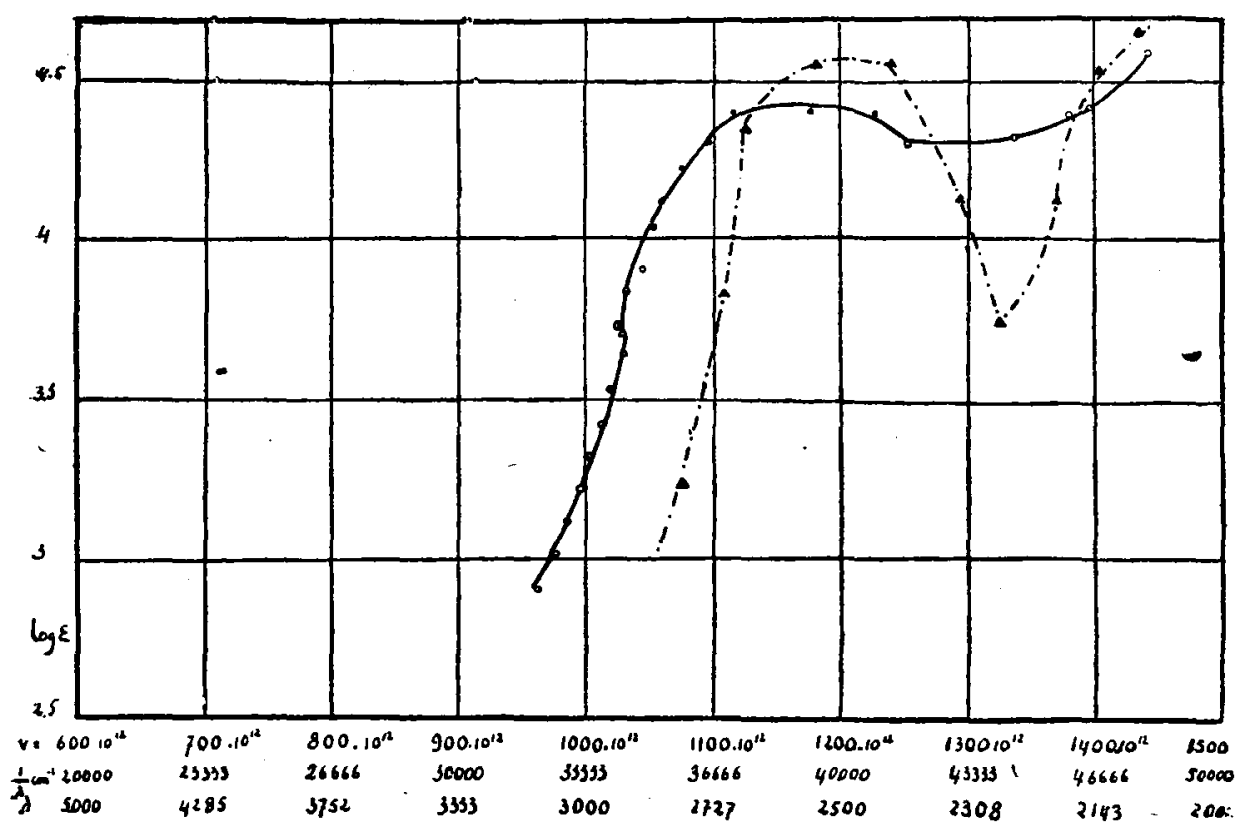

Fig. 12. - Spectre d’absorption du diphényle-dicéto-oyclobutane (énoligue)

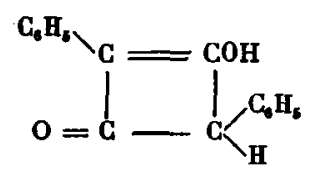

1. Trait plein : forme énolique dans l'éther.

2. Trait pointillé : forme dite cétonique dans l'hexane. 
On devrait obtenir par la réaction de Schroeter $\left({ }^{1}\right)$ l'éther éthylique de l'acide carbonique du monométhylecétène :<smiles>CCOC(=O)C(C)=C=O</smiles>

Nous ne sommes pas parvenus à isoler le corps simple mais bien son dimère, qui cristallise après refroidissement des liqueurs épaisses formées par les produits de décomposition. Son point de fusion est de 46,5 à $47,5^{\circ}$. Il est facilement soluble dans l'hexane.

Sa composition d'après l'analyse élémentaire est semblable à celle du cétène ${ }^{2}$ ). Son poids moléculaire théorique est égal à 256 . Nous avons trouvé par cryoscopie 241 et par ébullioscopie $258\left(^{3}\right)$.

$\mathrm{Si}$ on plonge la solution concentrée hexanique du dimère dans un mélange réfrigérant, neige de $\mathrm{CO}_{2}$-éther, il se précipite une huile qui est insoluble même dans l'hexane bouillant.

Cette huile abandonnée pendant un jour dans un local trais rēcristallise lentement soit en colonnes, soit en grandes plaques qui sont à nouveau solubles dans l'hexane.

Cette huile se dissout facilement dans l'éther, d'où elle recristallise sous la forme première par évaporation à froid du solvant $(\bullet)$.

(1) $B ., 42 ; 2336$ (1909) el Staudinger et Hirzel, B., 49, p. 1978-1994 (1916).

(3) Ce travail a été fait par MM. Tripet et Gonset.

0,1976 grs de subs. donnent............. 0,4097 grs $\mathrm{CO}_{2}$ et 0,1155 grs de $\mathrm{H}_{2} \mathrm{O}$

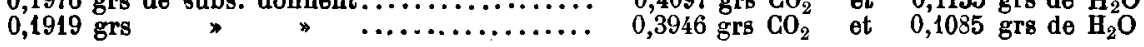

Trouvé :

$$
\text { Calcule pour } \mathrm{G}_{12} \mathrm{H}_{16} \mathrm{O}_{6}: \mathrm{G}=56,24 \% \text { et } \mathrm{H}=6,25 \%
$$$$
\begin{array}{llll}
\text { 1) } & \mathrm{C}=56,56 \% & \text { et } & \mathrm{H}=6,54 \% \\
\text { 2) } & \mathrm{C}=56,10 \% & \text { et } & \mathrm{H}=6,31 \%
\end{array}
$$

( ${ }^{3}$ D'après les expériences de M. Gonset : eryoscopie :

0,1606 grs. de subst. dissous dans 19,634 grs. de benzène donnent un abaissement du point de congélation de $0,18^{\circ} \mathrm{C}$. Poids moléculaire $=239$.

0,5088 grs. dissous dans 18,587 grs. de benzène donnent un $\Delta t=0,56^{\circ}$.

Poids moléculaire $=241$.

0,7308 grs. dissous dans 19,068 grs. de benzène donnent un $\Delta t=0,79^{\circ}$.

Ebullioscopie :

Poids moléculaire $=240$.

0,8788 grs. de subst. dissous dans 19,482 grs. de benzène produisent une élévation du point d'ébullition de $0,46^{\circ}$. Poids moléculaire $=260$.

1,4571 grs. de subst. dissous dans 19,482 grs. donnent un $\Delta t=0,77^{\circ}$.

Poids moléculaire $=258$.

(4) Cette transformation en une huile provient de la formation d'un isomère. Il y a deux possibilités : 


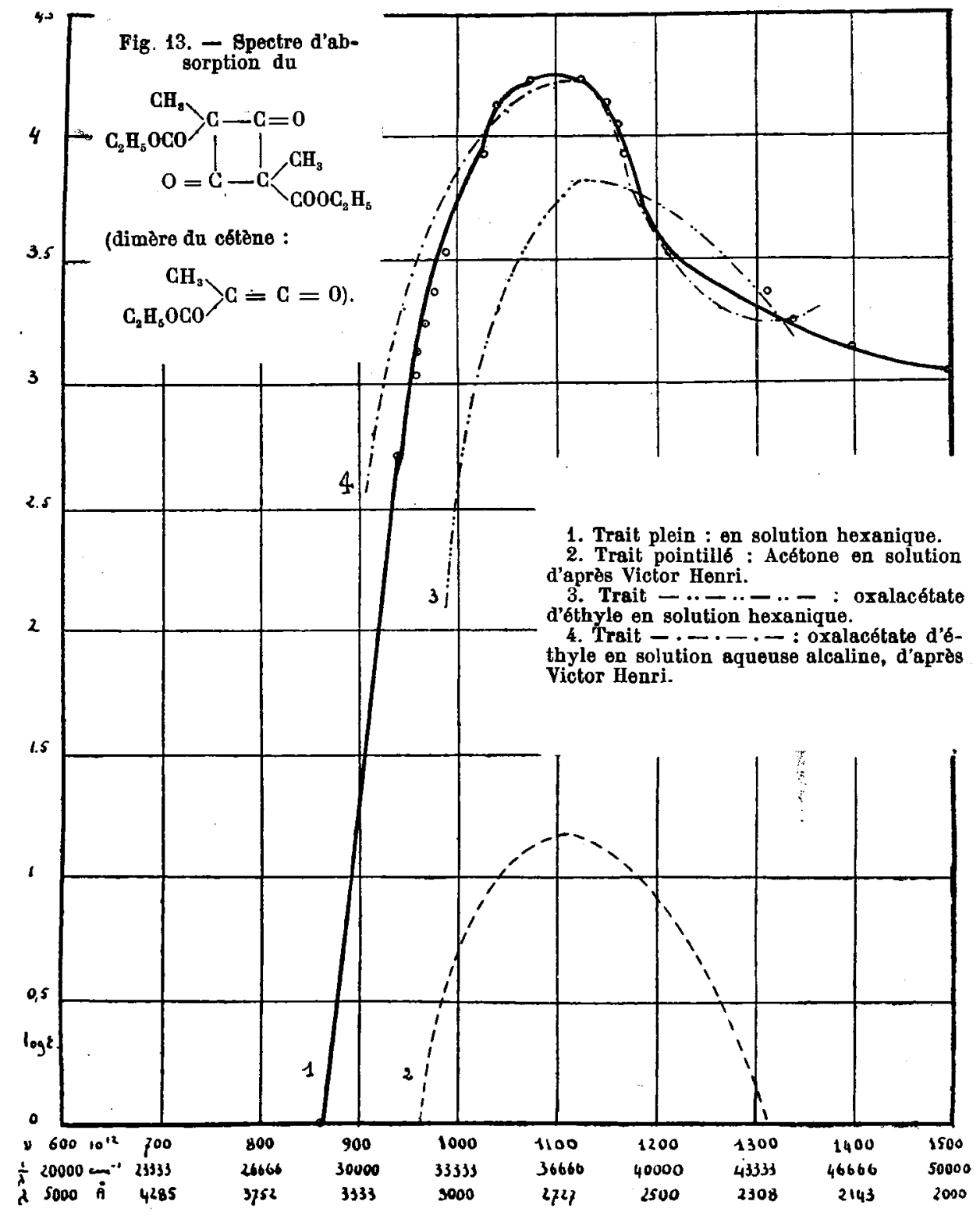


Spectrographie :

$0,4330 \mathrm{gr}$ (P. F. $46,6 \cdot 47,5^{\circ} \mathrm{C}$.) ont été dissous dans $33,81 \mathrm{~cm}^{2} \mathrm{~d}^{\prime}$ hexane (sol. $1 / 2 \mathrm{~mol} . / 10)$.

Nous avons photographié les spectres soit avec la lumière continue soit avec l'étincelle.

Les valeurs du coefficient d'absorption moléculaire sont les suivantes :

\begin{tabular}{|c|c|c|c|}
\hline$\lambda$ en $\AA$ & $\frac{1}{\lambda}$ en $\mathrm{cm}^{-2}$ & $v=\frac{3.10^{10}}{\lambda \mathrm{cm}}$ & $\varepsilon$ \\
\hline 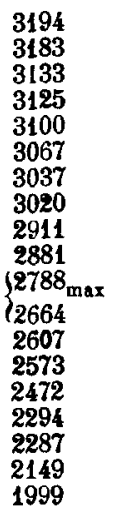 & $\begin{array}{c}31310 \\
31420 \\
31920 \\
32000 \\
32260 \\
32610 \\
32930 \\
33110 \\
34350 \\
34710 \\
35870 \text { max } \\
37540 \\
38360 \\
38870 \\
40450 \\
43590 \\
44500 \\
46530 \\
20005\end{array}$ & $\begin{array}{l}940.10^{12} \\
942 \\
958 \\
960 \\
968 \\
978 \\
988 \\
993 \\
1031 \\
1041 \\
1076_{\text {max }} \\
1126 \\
1150 \\
1166 \\
1213 \\
1307 \\
1335 \\
1395 \\
1501\end{array}$ & $\begin{array}{c}542 \\
704 \\
1084 \\
1400 \\
1744 \\
2232 \\
2710 \\
3504 \\
10840 \\
13242 \\
17440 \text { max } \\
17440 \\
13242 \\
10840 \\
3504 \\
2232 \\
1802 \\
1400 \\
1084\end{array}$ \\
\hline
\end{tabular}

Ou bien nous sommes en présence de deux stéréo-isomères cis et trans :

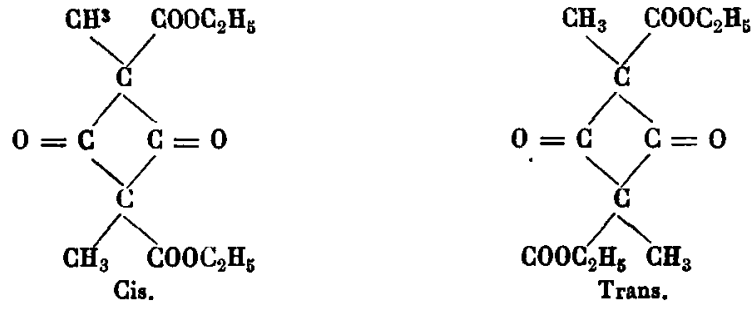

Ou bien c'est une isomérie céto-énolique, qui quoique rare dans ce cas n'est pas impossible.
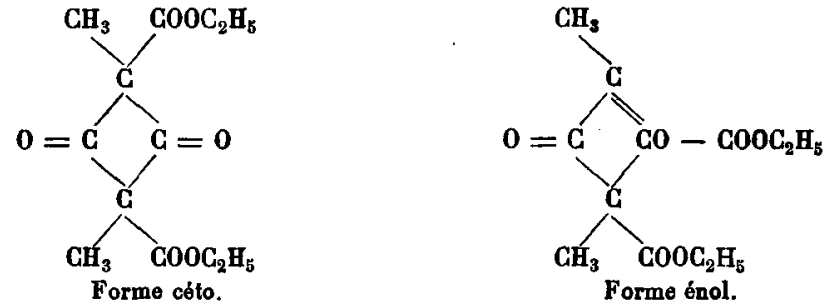

Le spectre d'absorption de notre produit en solution hexanique est superposable à celui de l'oxalacétate d'éthyle en solution alcaline. Ce spectre nous a été communiqué par M. Victor Henri. 
Le seul maximum que présente la courbe d'absorption de cette dicétone carboxylée se trouve aux mémes longueurs d'ondes que celui de l'acétone.

$$
\lambda_{\max }=2726 \AA \text { env. } \quad v=1101.10^{12} \quad \varepsilon=17440
$$

Pour l'acétone.

$$
\lambda_{\max }=2706 \quad \text { et } \quad v_{\max }=1109.10^{12} .
$$

La valeur de $\varepsilon$ est 600 fois plus forte environ mais la forme de la courbe de l'acétone n'est pas exactement reproduite.

La largeur de la bande est égale à $\Delta \nu=145.10^{12}$. Elle est symétrique $\left(^{1}\right)$.

\section{6. - SPECTRES D'ABSORPTION DES DERIV ES}

\section{DU DICETOCYCLOBUTANE N'EXISTANT QUE SOOS LA FORME ENOLIQUE (CY CLOB UTENOLONES)}

\section{Aci-diméthyledicétocyclobutane.}<smiles>CC1C2(C)C(=O)C1(C)C2O</smiles>

Ce produit préparé par Schroeter a été obligeamment mis à notre disposition par M. Staudinger. Nous l'en remercions.

Comme il n'y en avait fort peu et que le point de fusion était exact $\left(139^{\circ}-140^{\circ} \mathrm{C}\right.$.) nous avons préparé directement les solutions pour la spectrographie.

I) Solution aqueuse :

0,2433 grs ont été dissous dans $21,72 \mathrm{~cm}^{2}$ d'eau (sol. $\mathrm{mol} / 10$ ).

Nous avons photographié le spectre d'étincelle.

\begin{tabular}{|c|c|c|c|}
\hline$\lambda$ on $\AA$ & $\frac{I}{\lambda}$ en cm & $v=\frac{3.1010}{\lambda \mathrm{cm}}$ & $\varepsilon$ \\
\hline $\begin{array}{l}4072 \\
3360 \\
3020 \\
2813 \\
2714 \\
2627_{\text {max }} \\
2563 \\
2383 \\
2315 \\
2190 \\
2055 \\
1999\end{array}$ & $\begin{array}{c}24550 \\
29760 \\
31110 \\
35555 \\
36850 \\
38070_{\text {max }} \\
39020 \\
41960 \\
43200 \\
45600 \\
46660 \\
50020\end{array}$ & $\begin{array}{l}737.10^{12} \\
892 \\
993 \\
1066 \\
1105 \\
1142_{\max } \\
1170 \\
1258 \\
1295 \\
1369 \\
1459 \\
1501\end{array}$ & $\begin{array}{r}0,54 \\
3,6 \\
35,2 \\
3617 \\
11660 \\
\{17520 \text { max } \\
17520 \\
8720 \\
3520 \\
1808 \\
1808 \\
2711\end{array}$ \\
\hline
\end{tabular}

Les valeurs $\varepsilon$ sont les suivantes :

(1) Schroeter et Stassen, B., 40, 1604 (1907). 


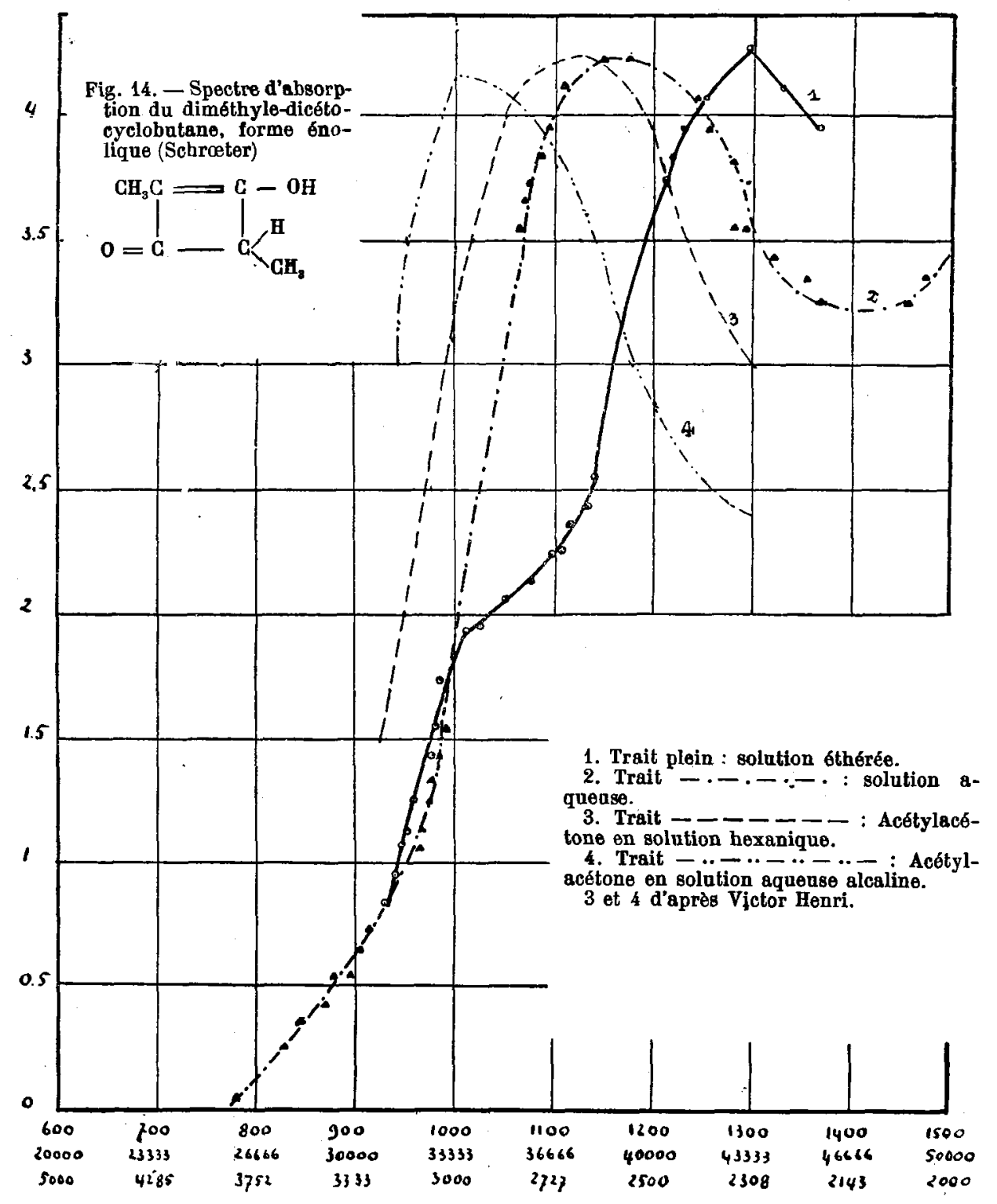


II) Solution éthérée :

$0,0673 \mathrm{grs}$ (regagnés de la solution aqueuse P. F. 139,5-140 ${ }^{\circ} \mathrm{C}$.) sont dissous dans $6,01 \mathrm{~cm}^{3}$ d'éther (sol. mol./10).

Nous avons photographié le spectre d'étincelle.

La table suivante donne les valeurs du coefficient d'absorption molénulaire :

\begin{tabular}{|c|c|c|c|}
\hline$\lambda$ en $\AA$ & $\frac{1}{\pi}$ en cm & $\nu=\frac{3.1010}{\lambda \mathrm{cm}}$ & $\varepsilon$ \\
\hline $\begin{array}{l}3219 \\
3047 \\
2831 \\
2706_{\max } \\
2625 \\
2453 \\
2432 \\
2315_{\max } \\
2190\end{array}$ & $\begin{array}{l}31070 \\
32820 \\
35320 \\
36950_{\text {max }} \\
38100 \\
40770 \\
41120 \\
43200_{\text {max }} \\
45660\end{array}$ & $\begin{array}{l}932.10^{12} \\
984 \\
1060 \\
1108_{\max } \\
1142 \\
1223 \\
1233 \\
1296_{\max } \\
1369\end{array}$ & $\begin{array}{c}6,6 \\
54,2 \\
116 \\
180_{\max } \\
352 \\
5420 \\
8720 \\
17520_{\max } \\
8720\end{array}$ \\
\hline
\end{tabular}

L'absorption est représentée par une bande très intense pour

$$
\lambda_{\max }=2588 \quad \vee=1156.10^{12} \quad \varepsilon=17520
$$

dont la largeur est égale à $\Delta \nu=170.10^{12}$.

Pour la solution éthérée, la courbe présente deux bandes. L'une très intense a son maximum vers :

$$
\lambda=2315 \AA \quad v=1296.10^{12} \quad \varepsilon=17520 .
$$

C'est la bande de la solution aqueuse déplacée vers l'ultra-violet $\Delta \nu=140.10^{12}$. Sa largeur est de $\Delta \nu=137.10^{12}$. Elle est plus étroite que celle de la solution aqueuse.

La seconde moins intense et moins nette est représentée par l'inflexion de la courbe de la fréquence $\nu=984.10^{12}$ à la fréquence $\nu=1108.10^{12}$ où $\varepsilon$ est égal à 180.

Nous retrouvons cette même bande dans le dicétocyclobutane en solution éthérée avec un $\varepsilon=27,12$ donc 6 fois moins intense.

Ces deux bandes correspondent par leur fréquence à la bande de l'acétone dont le sommet se trouve vers $\nu=1109.10^{12}$ avec un coefficient moléculaire d'absorption égal à 15,8 .

Pour la solution aqueuse tout le spectre est déplacé vers le visible et cette dernière bande n'apparaît pas.

\section{7. - SPECTRES D'ABSORPTION POUR DES CORPS A STRUCTURE RAPPROCHE DE CELLE DES C ETERTS}

a) L'isocyanate d'éthyle $\left(^{1}\right)$.

Nous avons obtenu l'isccyanate d'ethyle avec un très bon rendement $(93 \%)$ en chauffant l'azide de propionyle $\mathrm{CH}_{3} \mathrm{CH}_{2} \mathrm{CON}_{3}$ dans de l'anisol. L'isocyanate dis-

(2) G. Schroeter, B., 42, 3357 (1909). 
tille directement. Nous ne sommes pas parvenus à le séparer de toutes traces de chlorure de propionyle (qui n'avait pas réagit complètement avec l'azohydrate de sodium) en le fractionnant dans un ballon de Ladenbourg.

Nos essais de le purifier par distillation sur un excès de $\mathrm{NaN}_{3}$, sur de la chaux vive, sur de la poudre de cuivre, de l'oxyde d'argent (fraîchement précipité), sur des morceaux de sodium n'ont abouti à rien. Nous avons même essayé avec du potassium mais la réaction a été si vive que l'isocyanate s'est décomposé.

Nous avons alors traité cet isocyanate impur avec de la quinoléîne. A froid ce fut sans succès. Nous fúmes obligés de chaufter l'isocyanate d'éthyle en présence de quinoléine pendant 1 ti heures à $100^{\circ} \mathrm{C}$ en tube scellé.

Nous avons séparé la quinoléïne par congélation et distillation. Trois fractions se sont produites, pour les points d'ébullition suivants :

$1^{\circ} 58,5^{\circ}-59,5^{\circ}$.

$2^{\circ} 59,5^{\circ}-60,5^{\circ}$.

$3^{\circ} 61^{\circ}$. un peu.

Les deux premières ne contenaient pas de halogène ; la troisième en contenait

Nous avons conservé pendant plusieurs mois ces préparations d'isocyanates dans des tubes soudés. Seules celles qui avaient été traitées avec de la quinoléine sont restées incolores.

Spectrographie. mol./10).

0,2785 grs (P. E. 59,25\%-59,50') sont dissous dans $38,88 \mathrm{~cm}^{3}$ d'hexane (sol.

0,3906 grs ont été dissous dans $5,01 \mathrm{~cm}^{2}$ d'hexane (sol. molaire).

Les photographies ont été prises avec l'étincelle.

L'absorption ne présente rien de caracléristique dans les régions du visible et de l'ultra-violet.

Les valeurs de $\varepsilon$ pour les différentes longueurs d'ondes sont les suivantes :

\begin{tabular}{|c|c|c|c|}
\hline$\lambda$ en $\AA$ & $\frac{1}{\lambda}$ en $\mathrm{cm}^{-1}$ & $\nu=\frac{3.1010}{\lambda \mathrm{cm}}$ & $\varepsilon$ \\
\hline $\begin{array}{l}2848 \\
2566 \\
2348 \\
2036\end{array}$ & $\begin{array}{l}35110 \\
38970 \\
42590 \\
49120\end{array}$ & $\begin{array}{l}1053.1012 \\
1169 \\
1277 \\
1473\end{array}$ & $\begin{array}{r}0,1 \\
11,1 \\
11,6 \\
111,6\end{array}$ \\
\hline
\end{tabular}

b) L'isocyanate de phényle :

Nous avons employé les produits de Kahlbaum et de Siegtried-Zofingue, quenous avons purifiés par distillation et par cristallisation dans l'hexane à basse température.

Spectrographie :

$0,3349 \mathrm{grs}$ (P. E. $51,75-52$ à $13 \mathrm{~mm}$. de Hg dè p.) sont dissous dans $28,12 \mathrm{~cm}^{2}$ d'hexane (sol. $\mathrm{mol} / \mathrm{10}$ ).

5,0021 grs dans $15,57 \mathrm{~cm}^{2}$ d'hexane (sol. $2 \times$ molaire). 


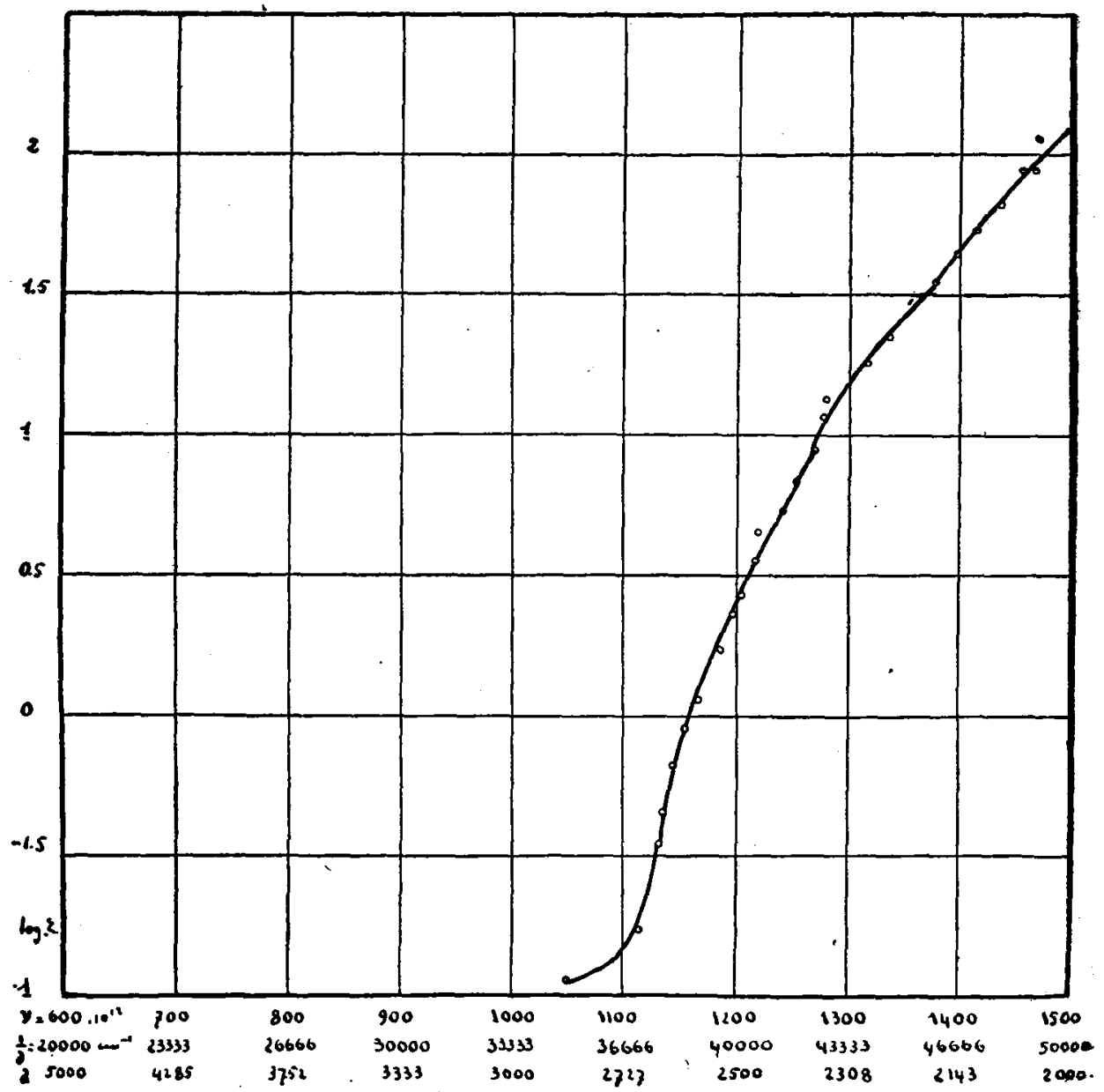

Fig. 15. - Spectre d'absorption de l'isocyanate d'éthyle $\mathrm{C}_{2} \mathrm{H}_{5} \mathrm{~N}=\mathrm{C}=0$.

Trait plein : en solution hexanique. 
Les valeurs de $\varepsilon$ sont les suivantes :

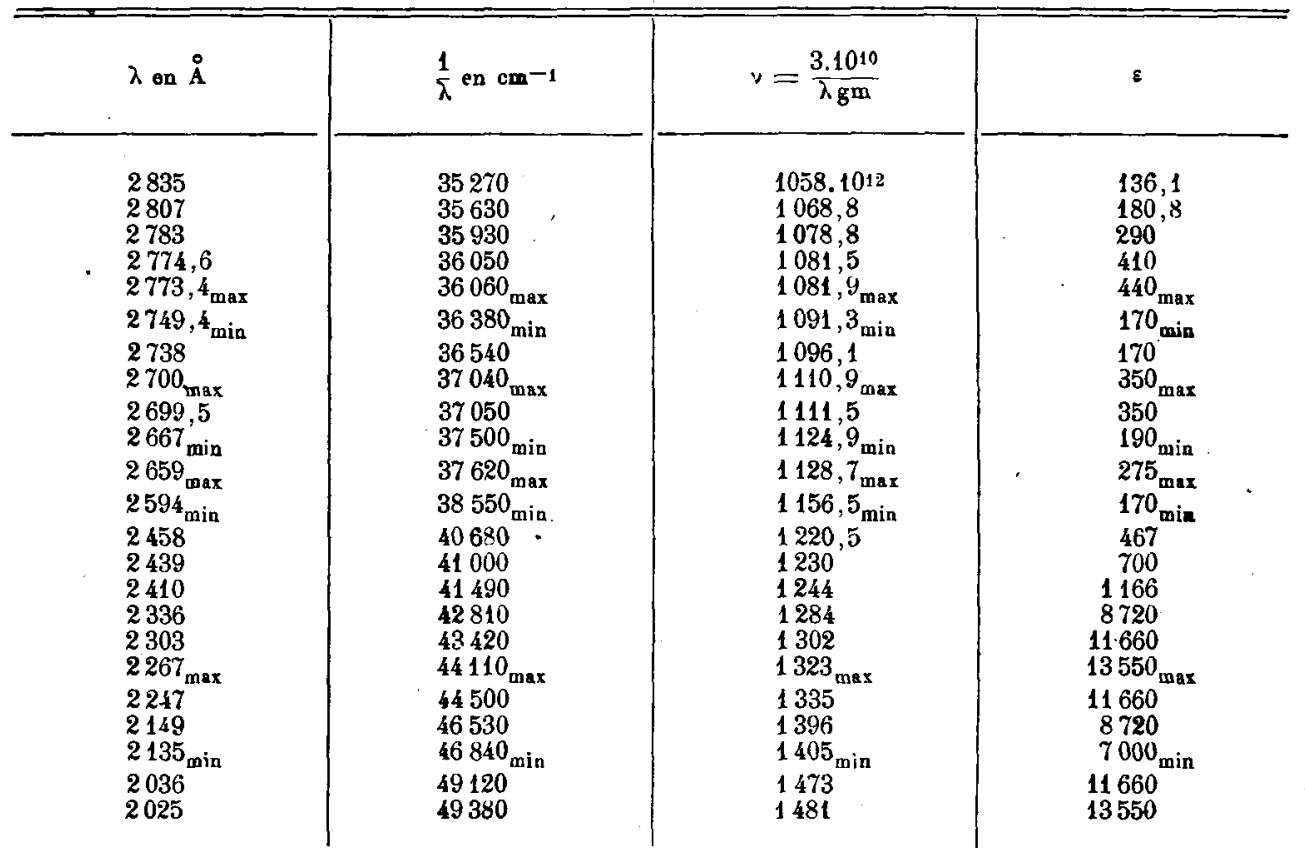

La courbe d'absorption présente deux régions caractéristiques : $1^{\circ}$ Trois bandes du type aromatique pour les fréquences :

$$
\begin{array}{lll}
\nu=1081,9.10^{12} & \lambda=2773,6 \AA & \varepsilon=440 \\
\nu=1111,2.10^{12} & \lambda=2700 \AA & \varepsilon=350 \\
\nu=1128,7.10^{12} & \lambda=2659 \AA & \varepsilon=275 .
\end{array}
$$

Pour le phénol Klingstedt $\left({ }^{1}\right)$ a observé les bandes suivantes qui sont très semblables par leur répartition à celles que nous avons déterminées pour l'isocyanate de phényle :

$$
\begin{array}{ll}
\nu=1082,3 \cdot 10^{12} & \varepsilon=2400 \\
\nu=1109,1.10^{12} & \varepsilon=2000 \\
\nu=1135,1.10^{12} & \varepsilon=1300 .
\end{array}
$$

Le rapport de leur intensité avec celle des bandes de l'isocyanate de phényle est d'environ 5.

$2^{\circ}$ Une bande large dans l'ultra-violet extrême dont le maximum se trouve vers :

$$
\lambda=2267 \AA \quad v=1323.10^{12} \quad \text { et } \quad \varepsilon=13550 .
$$

Sa largeur est égale à $\Delta \nu=136.10^{19}$.

(1) F. W. Kungstedt, C. R., t. 174, p. 812 (1922). 


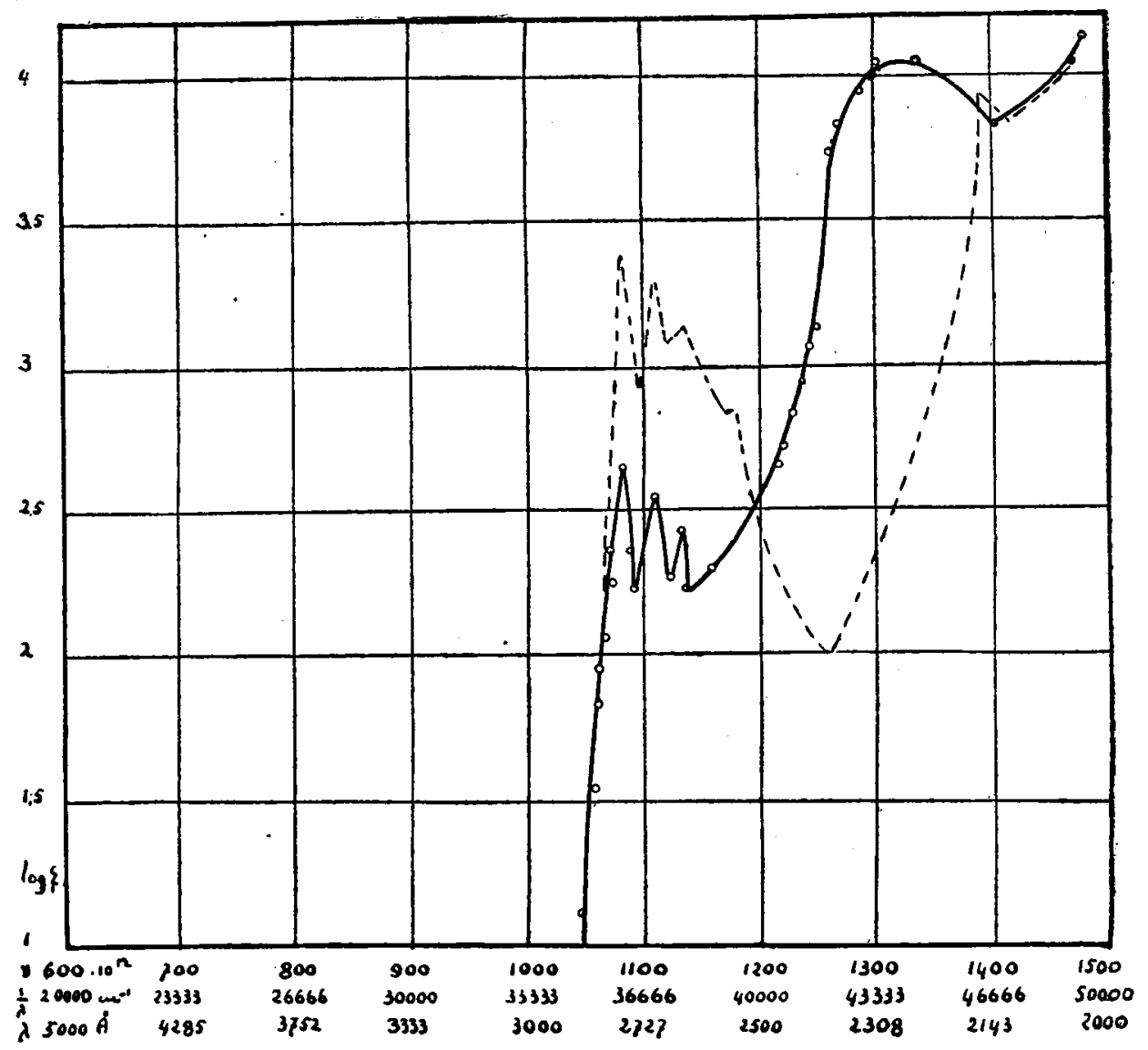

Fig. 16. - Spectre d'absorption de l'isocyanate de phényle $\mathrm{C}_{6} \mathrm{H}_{5} \mathrm{~N}=\mathrm{C}=0$.

1. Trait plein : en solution dans l'hexane.

2. Trait pointillé : phénol en solution heranique d'ạprès F. W. Klingstedt. 
Nous retrouvons cette bande dans toutes les combinaisons qui ont en position conjuguée d'un groupe phényle une liaison éthylénique, soit le diphényléthylène asymétrique, le diphénylecétène et le dyphényle-dicéto-cyclobutane (forme énolisée).

c) Le diéthyle-carbo-diimide $\mathrm{C}_{2} \mathrm{H}_{5} \mathrm{~N}=\mathrm{C}=\mathrm{NC}_{2} \mathrm{H}_{5}$.

Le diéthyle-carbo-diimide a été préparé en faisant réagir sur de l'éthylephosphimine de l'isosulfocyanate d'éthyle $\left(^{1}\right)$. La purification a été opérée par distillation.

$$
\begin{gathered}
\mathrm{C}_{2} \mathrm{H}_{5} \mathrm{~N}=\mathrm{P}\left(\mathrm{C}_{2} \mathrm{H}_{5}\right)_{3}+\mathrm{C}_{2} \mathrm{H}_{5} \mathrm{~N}=\mathrm{C}=\mathrm{S} \\
\mathrm{C}_{2} \mathrm{H}_{5} \mathrm{~N}-\mathrm{P}\left(\mathrm{C}_{2} \mathrm{H}_{5}\right)_{3} \\
\mathrm{C}_{2} \mathrm{H}_{5} \mathrm{~N}-\mathrm{N}=\mathrm{C}-\mathrm{S} \\
\mathrm{C}_{2} \mathrm{H}_{5} \mathrm{~N}=\mathrm{G}=\mathrm{NC}_{2} \mathrm{H}_{5}+\mathrm{S}=\mathrm{P}\left(\mathrm{C}_{2} \mathrm{H}_{5}\right)_{3}
\end{gathered}
$$

\begin{tabular}{|c|c|c|c|}
\hline$\lambda$ en $\AA$ & $\frac{1}{\lambda}$ en $\mathrm{cm}^{-1}$ & $\nu={ }_{\lambda}^{c} \mathrm{~cm}$ & $\varepsilon$ \\
\hline $\begin{array}{l}2888 \\
2324 \\
2800 \\
2701_{\max } \\
2600 \\
2545_{\max } \\
2336 \\
2210 \\
1979\end{array}$ & $\begin{array}{l}34690 \\
35410 \\
35710 \\
37020_{\max } \\
38480 \\
39310_{\text {max }} \\
42810 \\
45250 \\
50510\end{array}$ & $\begin{array}{l}1040.10^{12} \\
1062 \\
1071 \\
1110_{\max } \\
1153 \\
1179_{\max } \\
1273 \\
1357 \\
1515\end{array}$ & $\begin{array}{l}2,7 \\
9 \\
23,3 \\
27,1_{\text {max }} \\
27,1 \\
188_{\min } \\
135,5 \\
352 \\
1808\end{array}$ \\
\hline
\end{tabular}

0,1885 grs ont été dissous dans $19,24 \mathrm{~cm}^{3}$ d'hexane (sol. mol/10).

Nous avons mesuré pour $\varepsilon$ les valeurs suivantes :

La courbe d'absorption est caractérisée par une bande dont le maximum se trouve entre $\nu=1110.10^{22}$ et $\nu=1153.10^{12}$ avec un coefficient moléculaire d'absorption $\varepsilon=27,1$.

La largeur de la bande est égale à $\Delta \nu=130.10^{12}$.

On voit ici réapparaître, comme pour les azides, la bande qui a disparu dans le spectre ultra-violet des isocyanates.

d) Le diacétyle.

Nous avons préparé le diacétyle par la méthode ordinaire. Au lieu de le séparer de sa solution aqueuse par une distillation à la vapeur d'eau nous l'avons aspiré par le vide d'une trompe à eau. Les vapeurs de diacétyle étaient condensées

(1) Travail de M. C. de Lippert selon Staudinger et Hauser, Helo. Chim. Acta, IV, p. 881 (1921). 


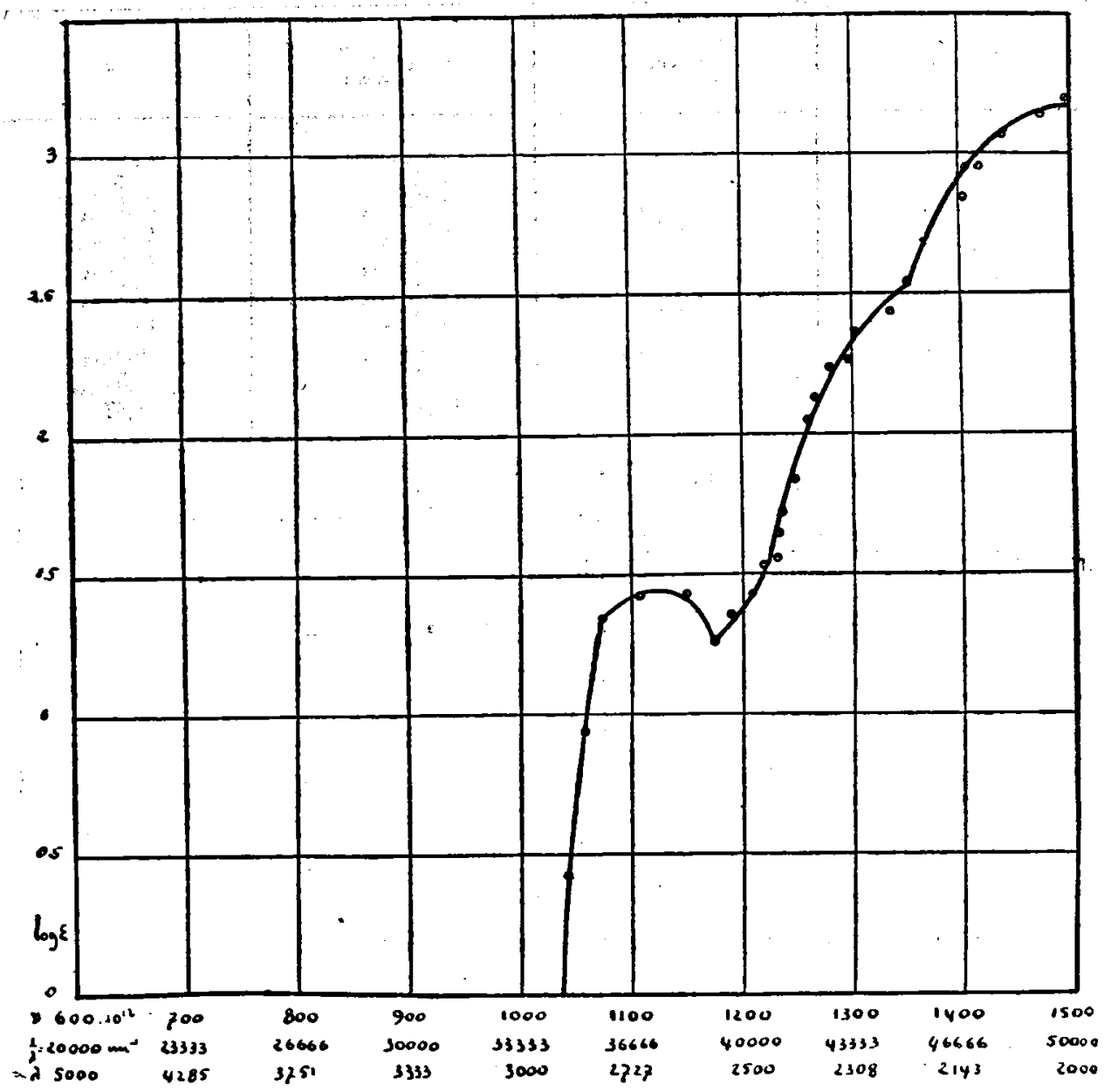

Fig. 17. - Spectre d'absorption du diéthyle-carbo-diimide $\mathrm{C}_{2} \mathrm{H}_{5} \mathrm{~N}=\mathrm{C}=\mathrm{NC}_{2} \mathrm{H}_{5}$.

Trait plein : on solntion hexanique. 
dans un mélange réfrigérant éther-neige de $\mathrm{CO}_{2}$. Nous l'avons purifié par distillation et cristallisation à $-40 \circ \mathrm{C}$.

$0,2463 \mathrm{grs}$ ont été dissous dans $28,6 \mathrm{~cm}^{2}$ d'hexane.

Voici les valeurs que nous avons trouvées pour $\varepsilon$ :

\begin{tabular}{|c|c|c|c|}
\hline$\lambda$ on $i$ & $\frac{1}{\lambda}$ on $\mathrm{cm}^{-1}$ & $\nu=\frac{3.1010}{\lambda \mathrm{cm}}$ & ' \\
\hline $\begin{array}{l}4535 \\
4504 \\
4194_{\max } \\
4434_{\max } \\
4245_{\max } \\
4237_{\max } \\
4051 \text { env. } \\
3337_{\max } \\
2813_{\max } \\
2445\end{array}$ & $\begin{array}{l}22058 \\
22200 \\
22250_{\text {max }} \\
22550_{\text {max }} \\
22550_{\text {max }} \\
23600_{\text {max }} \\
24685_{\text {max }} \\
29970_{\text {mix }} \\
35550_{\text {max }} \\
40900^{2}\end{array}$ & $\begin{array}{l}661,5.101= \\
666,1 \\
667,6_{\max } \\
6766_{\max } \\
706,7_{\max } \\
708,1_{\max } \\
7400_{\max } \\
899,0_{\min } \\
1066,4_{\max } \\
1227,0\end{array}$ & $\begin{array}{l}12 \\
20,5 \\
20,5_{\max } \\
18,85_{\max } \\
19,5_{\max } \\
19,5_{\max } \\
15_{\max } \\
0,5_{\min } \\
13,55_{\max } \\
11,67\end{array}$ \\
\hline
\end{tabular}

La courbe se compose de deux larges bandes.

La première entre $\lambda=4535 \AA$ et $\lambda=4051 \AA$ se subdivise en bandes étroites; nous avons pu déterminer 4 maxima :

$$
\begin{array}{lll}
\lambda=4494 \AA & \vee=667,6.10^{1 \mathrm{~s}} & \varepsilon=20,5 \\
\lambda=4434 \AA & \vee=676 & \varepsilon=18,8 \\
\lambda=4245 \AA & v=706,7 & \varepsilon=19,5 \\
\lambda=4051 \AA & \nu=740 \text { env. } & \varepsilon=15 \text { env. }
\end{array}
$$

La seconde qui a son maximum vers $\nu=1066,10^{12}$ avec $\varepsilon=13.55$ décrôtt très lentement vers l'ultra-violet. Comme position et intensité elle est très semblable à celle de l'acétone.

Le déplacement de la première bande vers les plus grandes longueurs d'ondes est dû à la position très rapprochées des deux chromophores. Elle se retrouve dans le glyoxal $\left({ }^{2}\right)$, le paraquinone $\left({ }^{2}\right)$ et le benzile $\left({ }^{3}\right)$, avec une subdivision plus fine, plus nette et plus régulièrement ordonnée. La bande du glyoxale se divise en 17 bandes fines, celle du paraquinone en 14 .

Cette bande à la lisière du visible est caractéristique pour les $\alpha$-dicétones. Son déplacement vers les plus grandes longueurs d'ondes est de $\Delta \nu=406.10^{\text {t5 }}$ par rapport à la bande de l'acétone. Ceci concorde avec sa non-saturation et sa facilité à polymériser.

Elle est reliée par sa fréquence moyenne $\nu=703.10^{12}$ à la bande infra-rouge

(2) Luthy, C. R., t. 176, p. 1547 (1923).

(2) F. W. Klingstedt, C.R., t. 176, p. 1550 (1923).

(3) D'après les résultats qui nous ont été communiqués par M. Armand Castille. 


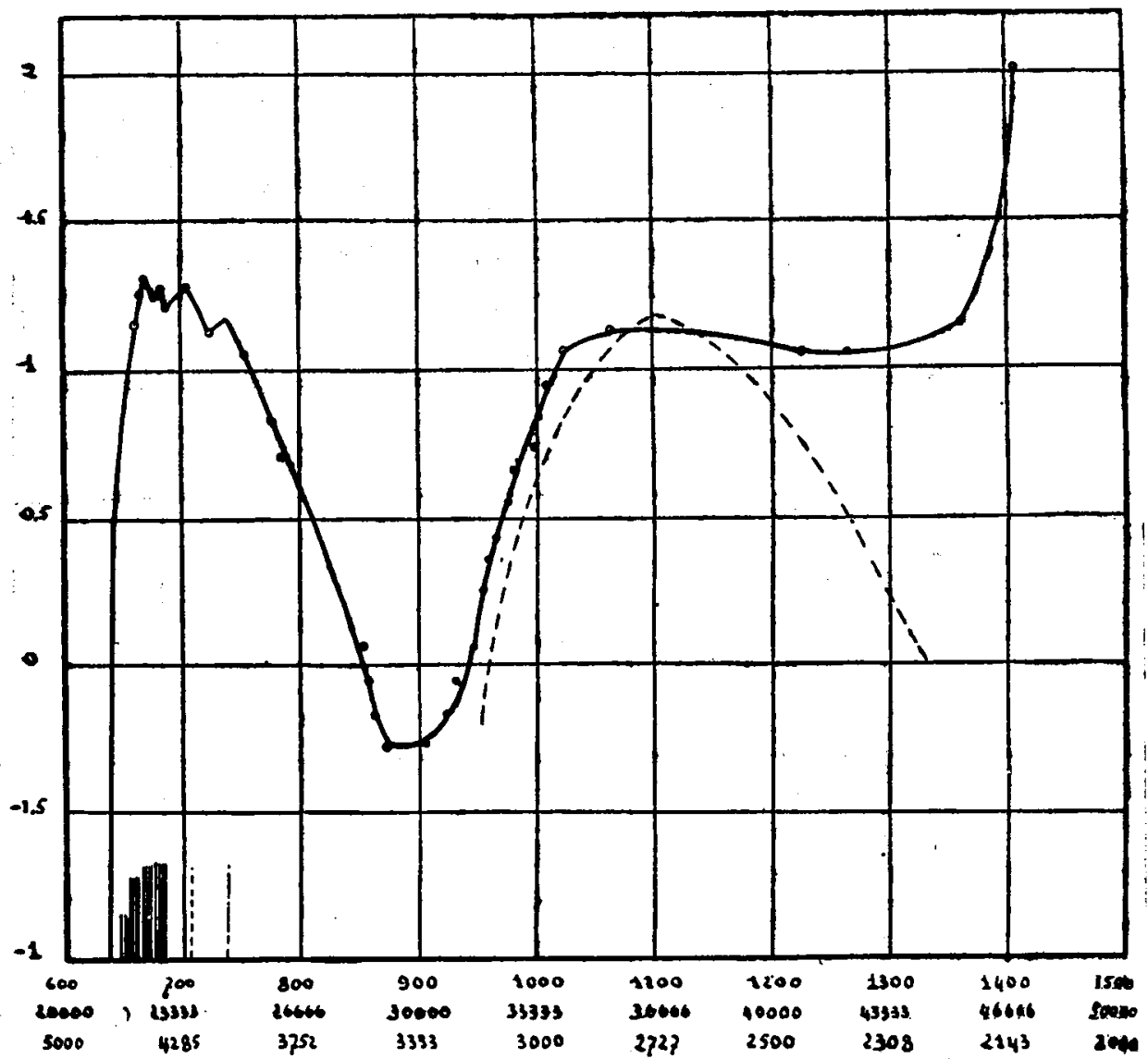

Fis. 18. - Spectre dabsorption dn diacśtyle.

1. Trait plein : en solntion heranique.

2. Trait pointillé : Acótone d'après Vietor Henri.

3. IN traits an bas de la planohe représentent les bandes du spectre du diacétyle l'état de rapeur; leur hanteur est proportionnelle à lour intensits relative. 
$a$ de l'acétone par une équation semblable à celle que nous avons observée pour les $\beta$-dicétones :

$$
v_{a}{ }^{\prime} \times 30=v_{\mathbf{i}, \text { v. }} \text { diacétyle } ; \quad 23,7.10^{12} \times 30=711.10^{12} .
$$

Spectre du diacétyle à l'état de vapeur :

Les quatre bandes étroites que nous avons mesurées pour le diacétyle en solution se subdivisent en bandes fines dans le spectre de la vapeur. Celles-ci sont très peu nettes et nous n'avons pas pu toutes les déterminer. Dans la région où se trouvent les deux premières bandes étroites de la solution $(4494 \AA$ et $4434 \AA$ ) nous en avons observées 15. Elles se groupent en quatre séries selon que leur bord fixe est du côté de l'ultra-violet ou du visible. Deux de ces séries se dégradent vers l'ultra-violet, ce sont les 2 et 4 ; les deux autres, 1 et 3 , se dégradent vers le rouge. Les séries de sens inverse s'alternent.

\begin{tabular}{|c|c|c|c|c|c|}
\hline & $\lambda$ en $\AA$ & $\frac{1}{\lambda}$ en $\mathrm{cm}^{-1}$ & $\Delta \frac{1}{\lambda}$ & $\nu=\frac{\mathbf{c m} / \mathbf{s e c}}{\lambda \mathbf{c m}}$ & $\Delta v$ \\
\hline $\begin{array}{c}1 \text { :o série } \\
a^{\prime \prime \prime} \\
b^{\prime \prime \prime} \\
c^{\prime \prime \prime} \\
d^{\prime \prime \prime}\end{array}$ & $\begin{array}{l}\ldots . . \\
4627 \\
4606 \\
4586\end{array}$ & $\begin{array}{c}\ldots . . \\
21610 \\
21710 \\
21805\end{array}$ & $\begin{array}{l}\cdots \\
100 \\
95\end{array}$ & $\begin{array}{c}\ldots . . \\
648,4,1012 \\
651.5 \\
654,2\end{array}$ & $\begin{array}{c}\cdots \\
3,1 \\
2,7\end{array}$ \\
\hline $\begin{array}{c}20 \text { série } \\
a^{\prime \prime} \\
b^{\prime \prime} \\
a^{\prime \prime} \\
d^{\prime \prime}\end{array}$ & $\begin{array}{l}45,78 \\
4560,5 \\
4545 \\
4532,5\end{array}$ & $\begin{array}{l}21845 \\
21930 \\
22000 \\
22065\end{array}$ & $\begin{array}{l}85 \\
70 \\
65\end{array}$ & $\begin{array}{l}655,3.10^{12} \\
657,0 \\
660,1 \\
662\end{array}$ & $\begin{array}{l}2.6 \\
242 \\
1,9\end{array}$ \\
\hline $\begin{array}{c}3^{\circ} \text { série } \\
a^{\prime} \\
b^{\prime} \\
c^{\prime} \\
d^{\prime}\end{array}$ & $\begin{array}{l}4499 \\
4481 \\
4461 \\
4442\end{array}$ & $\begin{array}{l}22215 \\
22315 \\
22415 \\
22510\end{array}$ & $\begin{array}{r}100 \\
100 \\
95\end{array}$ & $\begin{array}{l}666,8.10^{12} \\
669,5 \\
672 ' 5 \\
675.4\end{array}$ & $\begin{array}{l}2,7 \\
3 \\
2.9\end{array}$ \\
\hline $\begin{array}{c}\text { 40 série } \\
a \\
b \\
d\end{array}$ & $\begin{array}{l}4436 \\
4416 \\
4395 \\
4381\end{array}$ & $\begin{array}{l}22545 \\
22645 \\
22755 \\
22825\end{array}$ & $\begin{array}{r}100 \\
110 \\
70\end{array}$ & $\begin{array}{l}676,2.10^{12} \\
679,2 \\
682,6 \\
684.8\end{array}$ & $\begin{array}{l}3 \\
3,4 \\
2,2\end{array}$ \\
\hline
\end{tabular}


Une même relation que celle que nous avons observée entre les spectres de solution existe entre les spectres de vapeurs du glyoxal ( $\left.{ }^{2}\right)$ et du diacétyle. Pour le glyoxal les bandes sont plus nombreuses et plus nettes. Par l'introduction de groupes méthyles le spectre de vapeur des $\alpha$-dicétones se complique; les bandes fusionnent.

L'étude des spectres d'absorption des cétènes nous a permis d'arriver aux conclusions suivantes :

$1^{\circ}$ Le spectre d'absorption des cétènes est caractéristique. Il se compose de deux bandes larges. L'une, d'intensité moyenne se trouve dans le visible ou dans l'ultra-violet moyen. La seconde, d'intensité beaucoup plus forte, se trouve dans l'ultra-violet extrême ;

$2^{\circ}$ Par comparaison du spectre du diphénylecétène avec celui du diphényléthylène asymétrique, nous avons pu déterminer que la première bande du spectre des cétènes était due à la présence du groupe $\mathrm{C}=0$;

3o La deuxième bande dans l'ultra-violet extrême correspond à celle que Victor Henri a observé dans toutes les combinaisons contenant une liaison étbylénique ;

$4^{\circ}$ La molécule des cétènes ne contient pas un chromophore spécial mais bien les deux chromophores suivants:

$$
\begin{aligned}
& \text { 1) groupe } C=0 \\
& \text { 2) groupe } C=C \text {. }
\end{aligned}
$$

$5^{\circ}$ Il n'existe pas de différence entre les spectres d'absorption des aldocétènes du type $\mathrm{H}_{2} \mathrm{C}=\mathrm{G}=\mathrm{O}$ et les cétocétènes du type $\mathrm{R}_{2} \mathrm{C}=\mathrm{C}=\mathrm{O}$;

$6^{\circ}$ Une relation existe entre la tendance à polymériser des cétènes et la position de la première bande de leur spectre d'absorption.

Nous pouvons dire que le degré de non-saturation d'une molécule est déterminé par la position de ses bandes d'absorption.

Ceci est une contirmation de l'hypothèse de Staudinger sur la relation entre le renforcement de la couleur et la saturation d'une molécule.

$7^{\circ}$ Le diphénylecétène, dont la labilité, c'est-à-dire la vitesse avec laquelle il entre en réaction, est plus grande que celle des cétènes aliphatiques, se distingue par une intensité très torte de la bande du groupe $\mathrm{C}=\mathrm{O}$ de son spectre d'absorption. G'est en accord avec la loi de la labilité chimique et de l'intensité des bandes d'absorption de Victor Henri :

$8^{\circ}$ Le cétène $\mathrm{H}_{2} \mathrm{C}=\mathrm{C}=0$ à l'état de vapeur possède un spectre de bandes fines. Sa molécule est un dipôle. La présence de charges électriques explique selon Broenstedt les anomalies (autocatalyses) que nous observons dans la marche des réactions des cétènes ;

9o L'analogie des spectres d'absorption des cétènes, des diazoïques, des azides et des isocyanates, est en accord avec les observations de Staudinger sur leur nature chimique semblable;

(1) A. Luthy, Thèse, Zurich, 1923 (Jaboratoire de Victor Henri). 
10- Les cétènes se distinguent des isocyanates par la présence dans leur spectre d'absorption de la bande du groupe $\mathrm{C}=0$;

11. La différence entre le diméthyledicétocyclobutane énolique synthétisé par Sehroeter et les dicétones obtenues par polymérisation des cétocétènes est la même que celle qui existe entre le diphényledicétocyclobutane forme énolique et le diméthylediphényle-dicétocyclobutane. Ces deux dernières combinaisons ont été obtenues par polymérisation des cétènes;

120 Les dimères des cétènes possèdent les propriétés caractéristiques des $\beta$-dicétones. L'hypothèse qu'ils seraient des combinaisons dites a moléculaires a ne peut expliquer les faits expérimentaux ;

130 Le dimère du cétène $\mathrm{H}_{2}=\mathrm{C}=\mathrm{C}=\mathrm{O}$ est le' dicétocyclobutane. Il y a équilibre dans ses solutions entre les formes dicétoniques et énoliques ;

$14^{\circ}$ Le diphényledicétocyclobatane est monoénolisé en solution hexanique; la concentration en dicélone est de $1 \%$ à $13 \%$.

\section{CURRIGULUM VITE}

Originaire de Neuchâtel, Auvernier et Les Ponts de Martel (Canton de Neuchâtel), je suis né à Berne le 26 mars 1897, fils d'Edmond, doctear en médecine et d'Eugénie, née de Graffenried-Burgistein.

J'ai suivi comme élève régulier les classes du collège et du gymnase de Genève, qui me délivra en été 1916 le certificat de maturité de sa section classique.

Je fus alors immatriculé pendant un semestre à la faculté de Philosophie II (sciences) de l'université de Bâle.

En automne 1917, j'entrai comme élève régulier à l'Institut de chimie de l'Ecole Polytechnique Fédérale qui me décerna en mai 1921 le diplôme d'ingénieurchimiste (spécialisé dans l'électrochimie).

M. le Dr Staudinger, professeur de chimie générale à l'E. P. F. m'engagea alors comme assistant.

J'occupai mes loisirs à la préparation de cette thèse à laquelle j'ai travaillè sous sa baute direction et celle de $M$. le $D^{r}$ Victor Henri, professeur de chimie physique à l'Université de Zurich. 


\section{TABLE DES MATIÈRES}

Préface. - Généralités sur les cétènes et sur les spectres d'absorption.............

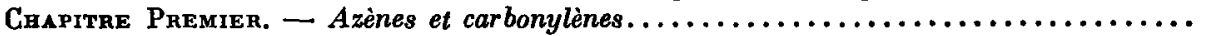

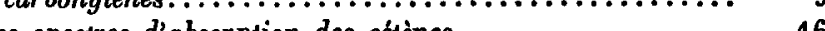

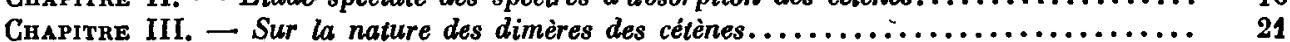

Partie expérimentale $: \ldots \ldots \ldots \ldots \ldots \ldots \ldots \ldots \ldots \ldots \ldots \ldots \ldots \ldots \ldots \ldots \ldots \ldots \ldots \ldots . . \ldots \ldots$

\$1. - Méthodes de travail ; Etablissement des courbes d'absorption ; Mesure

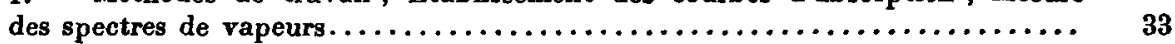

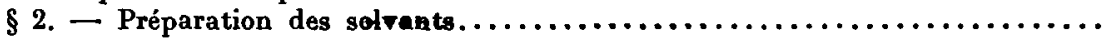

\$ 3. - Préparation et spectres d'absorption des cétènes : 1) cétène ; 2) diéthylecétène ; 3) dipropylecétène ; 4) diphénylecétène ; 5) diphényléthylène asymé-

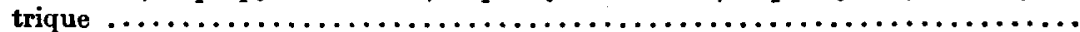

§4. - Préparation et spectres d'absorption des dimères des cétènes non-desmotropes : 1) tétraéthyledicétocyclobutane ; 2) tétrapropyledicétocyclobutane ; 3) tétra-allyledicétocyclobutane ;4) diméthylediphényledicétoclycobutane......

$\$ 5$. - Préparation et spectres d'absorption des dimères des cétènes avec fonction énolique possible : 1) dicétocyclobutane ; 2) diphényledicétocyclobutane (cétonique et énolique) ; 3) éther diéthylique de l'acide 1-3 dicarbonique du 1-3

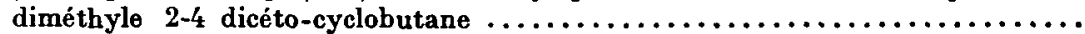

$\S 6$. - Spectres d'absorption des dérivés du dicétocyclobutane n'existant que sous la forme énolique : 1) diméthyle-dicétocyclobutane énolique (diméthyle-

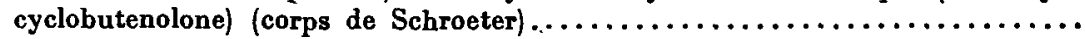

$\S 7$. - Préparation et spectres d'absorption des corps à structure rapprochée de celle des cétènes : 1) isocyanate d'éthyle ; 2) isocyanate de phényle ; 3) diéthyle-

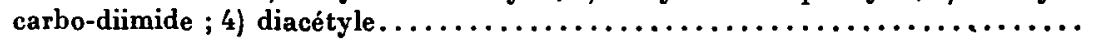




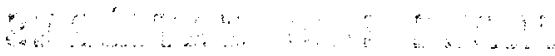

Iyprimern das Pazeses Univzhsitanzes, 49, Boulevard Saint-Michel, Paris. 834. 